\title{
DISCRETE ADAPTIVE RESPONSES TO MEK INHIBITOR IN SUBPOPULATIONS OF TRIPLE NEGATIVE BREAST CANCER
}

\author{
Daniel R. Goulet
}

A dissertation submitted to the faculty at the University of North Carolina at Chapel Hill in partial fulfillment of the requirements for the degree of Doctor of Philosophy in the Department of Pharmacology in the School of Medicine.

Chapel Hill

2018

Approved by:

Gary L. Johnson

Timothy C. Elston

Klaus M. Hahn

William Y. Kim

James E. Bear 
(C) 2018

Daniel R. Goulet

ALL RIGHTS RESERVED 


\section{ABSTRACT \\ Daniel R. Goulet: Discrete Adaptive Responses to MEK Inhibitor in Subpopulations of Triple Negative Breast Cancer (Under the direction of Gary Johnson)}

Triple-negative breast cancer (TNBC) is characterized by genomic heterogeneity and high risk of relapse. Recent studies have demonstrated that breast cancers can contain a spectrum of epithelial and mesenchymal phenotypes. We identified epithelial (POS) and mesenchymal (NEG) subpopulations in a triple negative breast cancer cell line that have similar coding sequences, but unique enrichment of the epigenetic activators H3K27ac and BRD4. We show that localization of these epigenetic activators correlates with the expression of epithelial transcription factors TP63 and ELF3 in epithelial cells, and ZEB1 and TWIST1 in mesenchymal cells. Currently, there are no targeted therapies available for the treatment of triple negative breast cancer. However, overexpression of EGFR, KRAS, or BRAF occurs in approximately $30 \%$ of TNBC, suggesting that inhibiting the MAPK signaling pathway may be an effective therapy for TNBC. Acute treatment with the MEK inhibitor trametinib induced distinct transcriptional responses in epithelial and mesenchymal subpopulations. ChIP-Seq analysis of H3K27ac and BRD4 binding revealed epigenetic remodeling in response to trametinib treatment, especially at binding sites for AP-1 in NEG cells and epithelial-specific ETS transcription factors in POS cells. Chronic treatment with trametinib induced the emergence of trametinib resistant POS cells, but not NEG cells. In trametinib resistant POS cells, we observe increased expression of the Gi-coupled chemokine receptor CXCR7, and its cognate ligand adrenomedullin, as well as increased expression of KRAS. ChIP-Seq analysis revealed enrichment of H3K27ac and BRD4 at the CXCR promoter and enhancer, indicating epigenetic remodeling induces transcriptional activation. siRNA knockdown of CXCR7 and KRAS demonstrate these genes are essential for the proliferation of trametinib resistant cells, and reveals that epigenetic remodeling at the CXCR7 locus may be a mechanism for drug resistance. 
To Jonathan King

who believed in me before I believed in myself 


\section{ACKOWLEDGEMENTS}

Firstly, l'd like to acknowledge my mentor, Professor Gary Johnson, without whose patience, guidance, and expertise this work would not have been possible. I'd like to thank my thesis committee of Professor Timothy Elston, Professors Klaus Hahn, James Bear, and William Kim for their thoughtful guidance and helpful suggestions throughout my graduate training. l'd like to acknowledge Dr. Jon Zawistowski, who has been an essential source of genomics knowledge and technical expertise. l'd like to acknowledge the contributions of the core facilities at the University of North Carolina that assisted with this work, especially Evan Trudeau at the Flow Cytometry Core, Yazhong Tao and Young Hu at the Translational Genomics Lab, and Nana Feinberg and Stephanie Cohen at the Translational Pathology Lab. I'd like to acknowledge all administrative staff in the Department of Pharmacology that make the department such a great place to work, especially: Chair of Pharmacology, Henrik Dohlman; Executive Assistant, Arlene Sandoval; Business Manager, Patty Holloway; Director of Graduate Studies, Lee

Graves; Information Technology, Vic Perez; Purchasing Manager, Alfred Dodge; and Student Services Manager, Nicole Arnold. I'd like to thank my co-workers and colleagues, past and present, for great conversation, friendship, and advice. Science is not an individual endeavor, and this work represents countless conversations with colleagues that helped refine my ideas and improve my technical skills. Finally, I'd like to acknowledge my family and friends whose unwavering support has sustained me throughout graduate school. 


\section{PREFACE}

Chapter 1 was written collaboratively with Samantha Bevill, and published in 2016 in the Journal of Cellular Physiology under the direction of Gary L. Johnson. The citation of the manuscript is: Miller, S.M. ${ }^{*}$, Goulet, D.R. ${ }^{*}$, Johnson, G.L. (2016). Targeting the Breast Cancer Kinome. Journal of Cellular Physiology. 232, 53-60. PMID: 27186656.

Sections titled "Intrinsic Resistance", "Acquired Resistance", and "Adaptive Resistance" in Chapter 3 were written collaboratively with Samantha Bevill, and published in 2016 in the Journal of Cellular Physiology under the direction of Gary L. Johnson. The citation for this work: Miller, S.M.*, Goulet, D.R.*, Johnson, G.L. (2016). Targeting the Breast Cancer Kinome. Journal of Cellular Physiology. 232, 53-60. PMID: 27186656. Figure 5, 6, and 7 were adapted from work published in collaboration with Zawistowski and Bevill et al. in Cancer Discovery in 2017. The citation for this work: Zawistowski, J.S. *, Bevill, S.M. *, Goulet, D.R., Stuhlmiller, T.J., Beltran, A.S., Olivares-Quintero, J.F., Singh, D., Sciaky, N., Parker, J.S., Rashid, N.U., Chen, X., Duncan, J.S., Whittle, M.C., Angus, S.P., Velarde, S.H., Golitz, B.T., He, X., Santos, C., Darr, D.B., Gallagher, K., Graves, L.M., Perou, C.M., Carey, L.A., Earp, H.S., Johnson, G.L. (2017). Enhancer Remodeling During Adaptive Bypass to MEK Inhibition is Attenuated by Pharmacological Targeting of the P-TEFb Complex. Cancer Discovery. 7, 302-321 (2017). PMID: 28108460 .

Chapter 4 is a work in preparation for submission as a research article in 2019 , under the direction of Gary L. Johnson. This work identifies epithelial and mesenchymal subpopulations in a triplenegative breast cancer cell line, SUM-229PE. The work demonstrates that these subpopulations are genetically similar but have distinct epigenetic profiles that determine gene expression in response to treatment with the MEK inhibitor trametinib. Chronic treatment with trametinib revealed the emergence of drug resistant cells in the epithelial subpopulation and identified KRAS and CXCR7 as key mediators of this resistance. 
This work was supported by the National Institutes of Health grants GM101141 (Awarded to GLJ) and CA0508223 (Awarded to GLJ), the Susan G. Komen Foundation grant IIR12-225201 (Awarded to GLJ), and the University Cancer Research Fund (Awarded to GLJ). 


\section{TABLE OF CONTENTS}

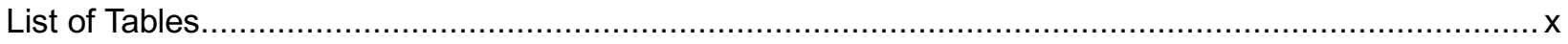

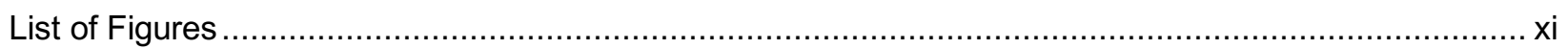

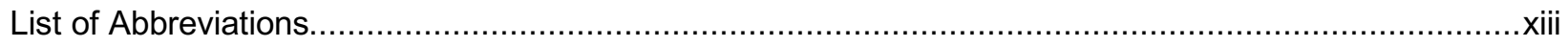

Chapter 1 : Breast Cancer Subtypes and Current Therapies ..................................................... 1

Genomic Analysis of the Breast Cancer Kinome .......................................................... 2

Kinase Inhibitors Targeting Breast Cancer Molecular Subtypes .......................................... 5

Chapter 2 : Human Transcriptional Regulation in Cancer ........................................................ 12

DNA Regulatory Elements in Mammalian Transcription .................................................. 12

Role of Chromatin Structure in the Regulation of Gene Expression ...................................... 14

Role of DNA Methylation in Transcriptional Regulation ...................................................... 15

Role of Histone Modifications in Transcriptional Regulation .............................................. 15

Epigenetic Dysregulation in Cancer ..................................................................... 17

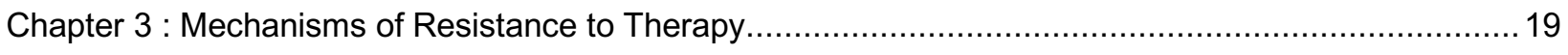

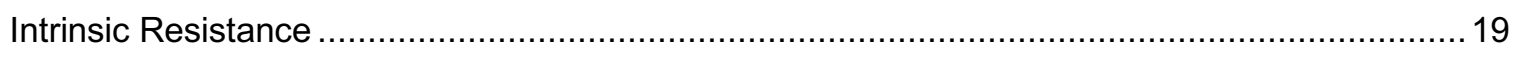

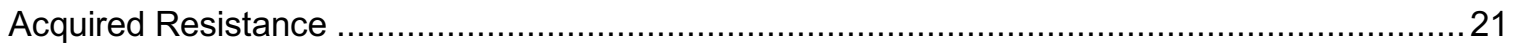

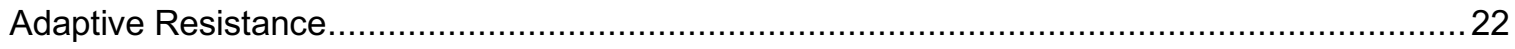

Subtype Specific Gene Expression in Response to Trametinib in TNBC …...........................24

Transcriptional Response to Trametinib is Associated with Epigenetic Remodeling ..................27

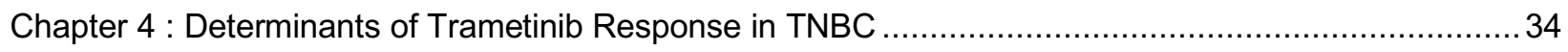

Subpopulations in TNBC Have Similar Genotypes with Distinct Phenotypes ........................... 36

Epithelial and Mesenchymal Phenotypes are Epigenetically Imprinted .................................. 42

Drug Response Determined by Differential Remodeling of Epigenetic Landscape.................... 44

Differential Response to Chronic Trametinib Treatment in Isolated Subpopulations ..................49

Epigenetic Remodeling Persists in Response to Chronic Trametinib Treatment ....................... 54 
Drug Resistant POS Cells Emerge Following Chronic Trametinib Treatment.......................... 56

Acquired Resistance and Epigenetic Remodeling Contribute to Trametinib Resistance.............61

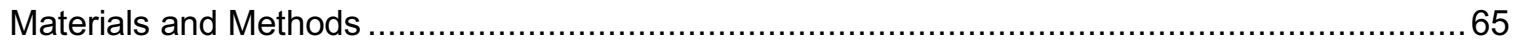

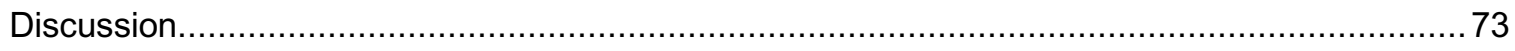

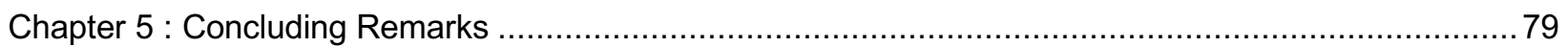




\section{LIST OF TABLES}

Table 1 - Targeted kinase inhibitors for treatment of breast cancer................................................... 7

Table 2 - Gene Expression of Differentially Mutated Genes Identified by Whole Exome Sequencing.

Table 3 - Markers Identified by Karyotyping of POS and NEG subpopulations. 


\section{LIST OF FIGURES}

Figure 1 - Overview of the Genomic Landscape Across Breast Cancer Molecular Subtypes

Figure 2 - Enhancer Regulation of Mammalian Transcription

Figure 3 - Intrinsic and Acquired Resistance Mechanisms in Breast Cancer

Figure 4 - Adaptive Resistance Mechanisms in Breast Cancer

Figure 5 - Unique Adaptive Response to Trametinib Treatment in Triple Negative

Breast Cancer Subtypes

Figure 6 - Transcriptional Response to Trametinib is Associated with Chromatin

Remodeling in TNBC

Figure 7 - Regulation of Target Gene Expression by Distal Enhancers 31

Figure 8 - Genetically Similar Subpopulations in TNBC Express Distinct Phenotypes

Figure 9 - Supplemental Figure : Genetically Similar Subpopulations in TNBC

Express Distinct Phenotype.....

Figure 10 - Epigenetic Imprinting in Epithelial and Mesenchymal Subpopulations

Figure 11 - Differential Transcriptional and Epigenetic Response to Acute Trametinib Treatment in Epithelial and Mesenchymal Cells

Figure 12 - Supplemental Figure : Differential Transcriptional and Epigenetic

Response to Acute Trametinib Treatment in Epithelial and Mesenchymal Cells

Figure 13 - Persistence of Acute Response in Chronic Treatment of Subpopulations

Figure 14 - Supplemental Figure : Persistence of Acute Response in Chronic Treatment of Subpopulations.

Figure 15 - Epigenetic Remodeling in Response to Chronic Trametinibe Treatment in Epithelial and Mesenchymal Subpopulations

Figure 16 - Resistant Cells Emerge in the Epithelial Subpopulation .57 
Figure 17 - Supplemental Figure : Resistant Cells Emerge in the Epithelial Subpopulation .......

Figure 18 - KRAS and CXCR7 Overexpression Contributes to Trametinib Resistance. 62

Figure 19 - Supplemental Figure : KRAS and CXCR7 Overexpression Contributes to Trametinib Resistance......

Figure 20 - Epigenetic Remodeling in Response to Trametinib Treatment in Subpopulations of Triple Negative Breast Cancer..... 


\section{LIST OF ABBREVIATIONS}

4EBP1

$\mathrm{ABL}$

ACTB

AKT

AP-1

AXL

BCL-2

BCR

BET

BEZ235

BKM120

BMS754807

bp

BRAF

BRD4

BSA

CBP

CCND1

CD49f

$\mathrm{CDH} 1$

CDK4

CDK6

CDKN2A

cDNA

CHD1

ChIP-Seq
Eukaryotic translation initiation factor 4E-binding protein 1

Abelson tyrosine-protein kinase 1

Beta actin

Protein kinase B

Activator protein 1

Tyrosine-protein kinase receptor UFO

B-cell lymphoma 2

Breakpoint cluster region protein

Bromo- and extra-terminal domain

PI3K Inhibitor Dactolisib

PI3K Inhibitor Buparlisib

IGF1R Inhibitor

Base pair

B-raf proto-oncogene

Bromodomain-containing protein 4

Bovine serum albumin

CREB-binding protein

Cyclin D1

Integrin alpha 6

E-Cadherin

Cyclin-dependent kinase 4

Cyclin-dependent kinase 6

Cyclin dependent kinase inhibitor 2A

Complementary DNA

Chromodomain helicase binding protein 1

Chromatin Immunoprecipitation Sequencing 


\begin{tabular}{|c|c|}
\hline $\mathrm{cm}$ & Centimeter \\
\hline CML & Chronic Myelogenous Leukemia \\
\hline CpG & Cytosine-Guanine dinucleotide \\
\hline CRISPR & Clustered Regularly Interspaced Short Palindromic Repeats \\
\hline CTCF & СССТC-binding factor \\
\hline CTD & C-terminal domain \\
\hline CXCR7 & Chemokine receptor 7 \\
\hline DCLK1 & Doublecortin-like kinase 1 \\
\hline DDR1 & Discoidin domain receptor 1 \\
\hline DDR2 & Discoidin domain receptor 2 \\
\hline DESeq & Differential gene expression package for $R$ \\
\hline DMSO & Dimethyl sulfoxide \\
\hline DNA & Deoxyribonucleic acid \\
\hline DNMT & DNA methyltransferase \\
\hline DNMT1 & DNA methyltransferase 1 \\
\hline DNMT3A & DNA methyltransferase $3 \mathrm{~A}$ \\
\hline DNMT3B & DNA methyltransferase 3B \\
\hline DSG2 & Desmoglein 2 \\
\hline EDTA & Ethylenediaminetetraacetic acid \\
\hline EGFR & Epidermal growth factor receptor \\
\hline ELF3 & E74-like ETS transcription factor 3 \\
\hline EpCAM & Epithelial cell adhesion molecule \\
\hline ER & Estrogen receptor \\
\hline ERBB & Erythroblastic leukemia viral oncogene \\
\hline ERK & Extracellular signal-regulated kinase \\
\hline ERK2 & Extracellular signal-regulated kinase 2 \\
\hline ETS-1 & Avian erythroblastosis virus E26 oncogene homolog 1 \\
\hline ETS-2 & Avian erythroblastosis virus E26 oncogene homolog 2 \\
\hline
\end{tabular}


Histone lysine N-methyltransferase 1

$\mathrm{EZH} 2$

Histone lysine N-methyltransferase 2

FACS

Fluorescence-activated cell sorting

FBS

Fetal bovine serum

FDA

Food and Drug Administration

FGFR

Fibroblast growth factor receptor

FGFR1

Fibroblast growth factor receptor 1

FGFR2

Fibroblast growth factor receptor 2

FGFR4

Fibroblast growth factor receptor 4

FN1

Fibronectin

FOSL2

Fos-related antigen 2

FOXA1

Forkhead box protein A1

FOXM1

Forkhead box protein M1

FOXO

Forkhead box protein $\mathrm{O}$

FRK

Fyn-related kinase

G1

GATA3

Gap 1 phase

GATA-binding protein 3

GO

Gene ontology

GSK2141795

AKT inhibitor

H3K27ac

Histone H3 lysine 27 acetylation

H3K27me3

Histone $\mathrm{H} 3$ lysine 27 trimethylation

H3K4

Histone H3 lysine 4

H3K4me1

Histone $\mathrm{H} 3$ monomethylation

H3K4me3

Histone $\mathrm{H} 3$ trimethylation

HCC-1806

Basal-like breast cancer cell line

HER2

Human epidermal growth factor receptor 2

HER3

Human epidermal growth factor receptor 3

HF

Ham's F12 Media + 2\% FBS + 5 mM EDTA 


\begin{tabular}{|c|c|}
\hline HMT & Histone methyltransferase \\
\hline HRP & Horseradish peroxidase \\
\hline HS-578T & Claudin-low breast cancer cell line \\
\hline IGF1R & Insulin growth factor receptor 1 \\
\hline INPP4B & Inositol phosphate-4 phosphatase type II B \\
\hline INSR & Insulin receptor \\
\hline IP6K3 & Inositol hexakisphosphate kinase 3 \\
\hline ITGA11 & Integrin alpha 11 \\
\hline ITGA6 & Integrin alpha 6 \\
\hline ITGB1 & Integrin beta 1 \\
\hline ITGB4 & Integrin beta 4 \\
\hline JAK1 & Janus kinase 1 \\
\hline JNK & Jun kinase \\
\hline JQ1 & BET bromodomain inhibitor \\
\hline JUND & Transcription factor jun-D \\
\hline $\mathrm{kb}$ & Kilobase \\
\hline KDM1A & Lysine demethylase $1 \mathrm{~A}$ \\
\hline KDM1B & Lysine demethylase 1B \\
\hline KDM5A & Lysine demethylase $5 \mathrm{~A}$ \\
\hline KDM5B & Lysine demethylase 5B \\
\hline KDM5C & Lysine demethylase 5C \\
\hline KDM5D & Lysine demethylase 5D \\
\hline KDR & Vascular endothelial growth factor receptor 2 \\
\hline $\mathrm{KIT}$ & Stem cell growth factor receptor \\
\hline KRAS & Kirsten Ras oncogene \\
\hline KRT18 & Cytokeratin 18 \\
\hline KRT5 & Cytokeratin 5 \\
\hline KRT7 & Cytokeratin 7 \\
\hline
\end{tabular}




\begin{tabular}{|c|c|}
\hline $\mathrm{LiCl}$ & Lithium chloride \\
\hline $\mathrm{mA}$ & Milliamp \\
\hline MAP & Mitogen-activated protein \\
\hline MAP2K4 & Mitogen-activated protein kinase kinase 4 \\
\hline MAP2K6 & Mitogen-activated protein kinase kinase 6 \\
\hline MAP3K1 & Mitogen-activated protein kinase kinase kinase 1 \\
\hline MAPK & Mitogen-activated protein kinase \\
\hline MDA-MB-468 & Basal-like breast cancer cell line \\
\hline MEK & MAPKJERK kinase \\
\hline MET & Hepatocyte growth factor receptor \\
\hline MK2206 & AKT inhibitor \\
\hline $\mathrm{mL}$ & Milliliter \\
\hline$\mu \mathrm{L}$ & Microliter \\
\hline$\mu \mathrm{g}$ & Microgram \\
\hline MLL1 & Histone lysine N-methyltransferase $2 \mathrm{~A}$ \\
\hline MLL2 & Histone lysine N-methyltransferase 2D \\
\hline MLL3 & Histone lysine $\mathrm{N}$-methyltransferase $2 \mathrm{C}$ \\
\hline MLL4 & Histone lysine N-methyltransferase 2B \\
\hline $\mathrm{mm}$ & Millimeter \\
\hline $\mathrm{mM}$ & Millimolar \\
\hline MOZ & Histone acetyltransferase $6 \mathrm{~A}$ \\
\hline mRNA & Messenger RNA \\
\hline MSigDB & Molecular Signature Database \\
\hline mTOR & Mammalian target of rapamycin \\
\hline mTORC1 & Mammalian target of rapamycin complex 1 \\
\hline MYC & Proto-oncogene c-Myc \\
\hline MYLK & Myosin light chain kinase \\
\hline $\mathrm{NaCl}$ & Sodium chloride \\
\hline
\end{tabular}




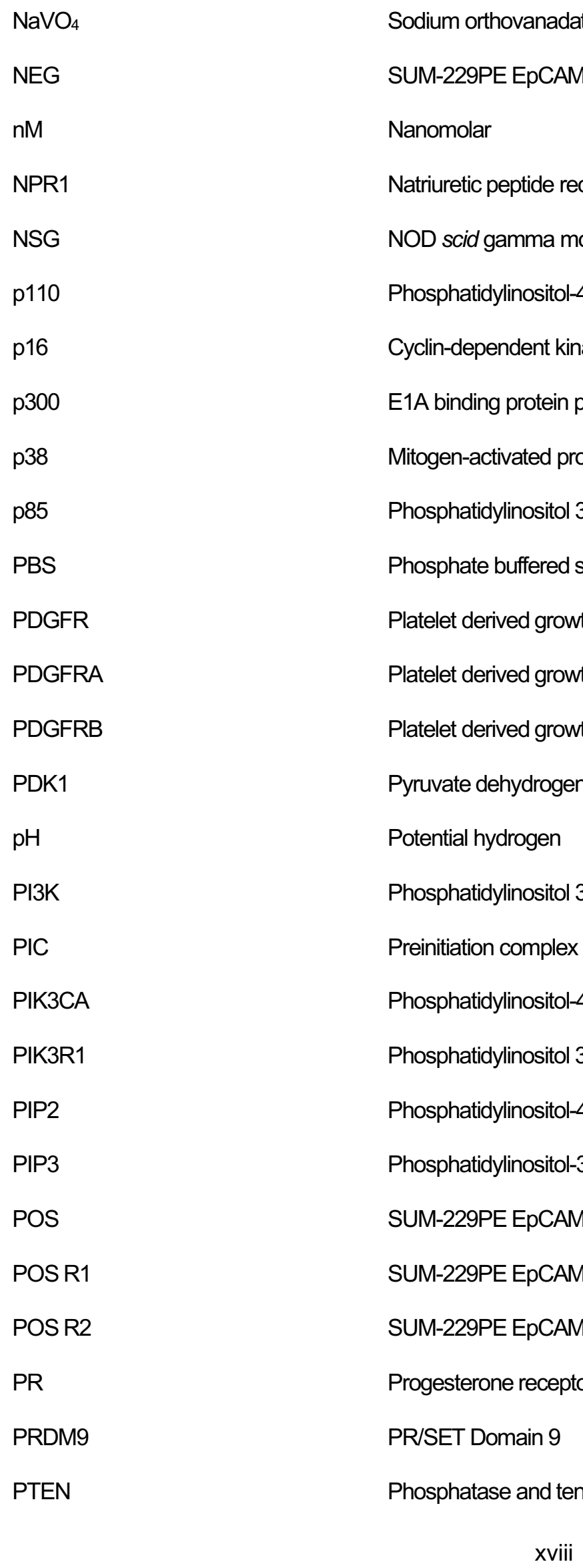




\begin{tabular}{|c|c|}
\hline RAF & c-Raf proto-oncogene, serine/threonine kinase \\
\hline RAS & GTPase proto-oncogene \\
\hline RIOX1 & Ribosomal oxygenase 1 \\
\hline RNA & Ribonucleic acid \\
\hline RNA-FISH & Ribonucleic acid fluorescence in situ hybridization \\
\hline RNA-Seq & Ribonucleic acid sequencing \\
\hline RNAPII & RNA polymerase II \\
\hline RPM & Revolutions per minute \\
\hline RPPA & Reverse phase protein array \\
\hline RTK & Receptor tyrosine kinase \\
\hline SDS-PAGE & Sodium dodecyl sulfate polyacrylamide gel electrophoresis \\
\hline SET & Lysine N-methyltransferase \\
\hline SET1A & Lysine N-methyltransferase $2 \mathrm{~F}$ \\
\hline SET1B & Lysine N-methyltransferase 2G \\
\hline SET7/9 & Lysine N-methyltransferase 7/9 \\
\hline sgRNA & Single guide ribonucleic acid \\
\hline siRNA & Small interfering ribonucleic acid \\
\hline SMARCD3 & $\begin{array}{l}\text { SWI/SNF-related matrix-associated actin-dependent regulator of chromatin } \\
\text { subfamily D member } 3\end{array}$ \\
\hline SMYD1 & SET and MYND Domain-Containing Protein 1 \\
\hline SMYD2 & SET and MYND Domain-Containing Protein 2 \\
\hline SNAI1 & Snail 1 \\
\hline SNV & Single nucleotide variant \\
\hline SRC & Proto-oncogene protein kinase Src \\
\hline SUM-149 & Basal-like breast cancer cell line \\
\hline SUM-159 & Claudin-low breast cancer cell line \\
\hline SUM-229PE & Triple-negative breast cancer cell line \\
\hline SWI/SNF & Switch/sucrose non-fermentable nucleosome remodeling complex \\
\hline
\end{tabular}




\begin{tabular}{|c|c|}
\hline TAD & Topological association domain \\
\hline TBST & Tris-buffered saline $+0.1 \%$ Tween- 20 \\
\hline TCGA & The Cancer Genome Atlas \\
\hline TES & Transcription end site \\
\hline TGF $\beta$ & Transforming growth factor beta \\
\hline TGFB1 & Transforming growth factor beta 1 \\
\hline TGFBR3 & Transforming growth factor beta receptor 3 \\
\hline TIF2 & Nuclear receptor coactivator 2 \\
\hline TNBC & Triple negative breast cancer \\
\hline TP53 & Tumor protein p53 \\
\hline TP63 & Tumor protein p63 \\
\hline Tram & Trametinib \\
\hline Tris-HCl & Tris(hydroxymethyl)aminomethane hydrochloride \\
\hline TSS & Transcription start site \\
\hline TWIST1 & Twist family BHLH transcription factor \\
\hline $\mathrm{V}$ & Volts \\
\hline VEGFA & Vascular endothelial growth factor alpha \\
\hline VEGFR & Vascular endothelial growth factor receptor \\
\hline VIM & Vimentin \\
\hline WHIM12 & Claudin-low breast cancer \\
\hline ZEB1 & Zinc-finger e-box binding homeobox 1 \\
\hline
\end{tabular}




\section{CHAPTER 1: BREAST CANCER SUBTYPES AND CURRENT THERAPIES}

Kinases have been recognized as highly tractable targets for cancer treatment due to their druggability and the critical roles they play in regulating cellular growth, differentiation, migration, and survival. There are currently 28 FDA-approved kinase inhibitors (P. Wu et al., 2016), with many more in various stages of clinical trials. Successful kinase inhibitor clinical trials in cancer patients have targeted kinases that are mutated or overexpressed and where additional preclinical evidence demonstrates that the target kinase is sufficient to drive disease pathology. For example, mutant BRAF in melanoma can be targeted with the FDA approved targeted kinase inhibitors vemurafenib and dabrafenib. Similarly, EGFR mutations are targeted in non-small cell lung tumors using the kinase inhibitors gefitinib and erlotinib. However, comprehensive sequencing studies have revealed that unlike many cancers, the landscape of the breast cancer genome is characterized by few oncogenic mutations, and is instead dominated by copy number variants and somatic copy number aberrations. Whole exome sequencing of 507 breast tumors by the TCGA identified somatic mutations in only three genes with greater than $10 \%$ incidence rate: TP53, PIK3CA, and GATA3 (Cancer Genome Atlas Network, 2012). Analysis of 2,000 breast tumors demonstrated that somatic copy number aberrations alter expression of approximately $40 \%$ of tumor genes (Curtis et al., 2012), but the role of these copy number variants and somatic copy number aberrations in tumorigenesis remains unclear. Therefore, identifying candidate kinases to target in breast tumors has proven difficult given the lack of dominating mutations in signaling pathways that have the potential to drive disease progression.

Breast cancer is a heterogeneous disease and molecular characterization of tumors has defined five distinct subtypes that are associated with different prognoses including luminal A, luminal B, HER2enriched, basal-like and claudin-low (Perou et al., 2000; Prat et al., 2010). Interrogation of the breast cancer genome and transcriptome has identified genes that are uniquely altered across the molecular subtypes that represent potential targets for therapeutic intervention. For instance, luminal A and B subtypes typically express the estrogen $(E R)$ and/or progesterone $(P R)$ receptors which can be targeted 
with hormone therapy. Additionally, notable success has been achieved in HER2-enriched tumors by targeting the HER2 receptor tyrosine kinase (RTK) with monoclonal antibodies and small molecule inhibitors. However, basal-like and claudin-low subtypes are defined as triple negative breast cancer (TNBC) due to the absence of targetable ER, PR, and HER2 receptor expression. A multiplatform analysis across over 3,500 specimens and twelve tumor types found that breast cancer specimens fell into two groups where luminal and HER2-enriched tumors clustered away from basal-like specimens (Hoadley et al. 2014). Furthermore, comparison across all twelve tumor types revealed that basal-like tumors were more similar to lung cancer than they were to luminal and HER2-enriched tumors. These data support the notion that triple negative breast cancer encompasses a unique disease state. This vast heterogeneity of breast cancer has deterred the identification of targetable kinases driving disease pathology across breast cancer, and as a result ongoing studies are focusing on defining therapeutic targets within each subtype of breast cancer in order to develop customized therapies for each subtype. This chapter discusses the genomic landscape of the kinome in breast cancer subtypes, kinase inhibitors being investigated in clinical trials.

\section{Genomic Analysis of the Breast Cancer Kinome}

\section{Luminal Breast Cancer}

Tumors classified in the luminal A molecular subtype contain the fewest defined mutations, but exhibit high mutational frequency in a select number of genes including PIK3CA at $44 \%$, followed by MAP3K1 (13\%), and MAP2K4 (7\%) (Figure 1A and B). Luminal A tumors are typically dependent on ER activation for proliferation, and are responsive to hormone therapy. Pathway analysis of luminal breast cancer revealed FOXA1-ER as an essential signaling hub in luminal tumors, and hazard models indicate that low expression of FOXA1 improves prognosis in luminal A breast cancer patients (Badve et al., 2007). High frequency of PIK3CA mutations suggests activation of the AKT signaling pathway, but PIK3CA mutations do not significantly increase phosphorylation of biomarkers for PIK3CA activation including AKT, ribosomal protein S6 or 4EBP1, suggesting compensatory feedback regulatory mechanisms are controlling activity of the pathway in luminal breast cancers. This contrasts with PIK3CA mutation or amplification in HER2-enriched tumors and PTEN or INPP4B loss in basal breast cancers, 
A. Luminal A/B

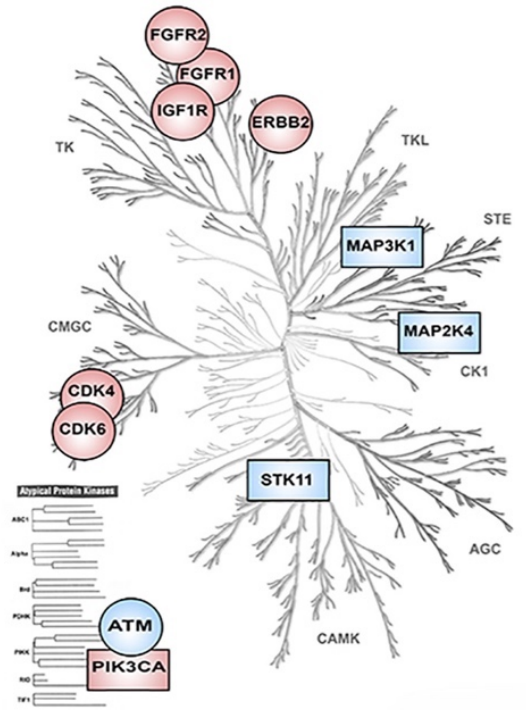

HER2-Amplified

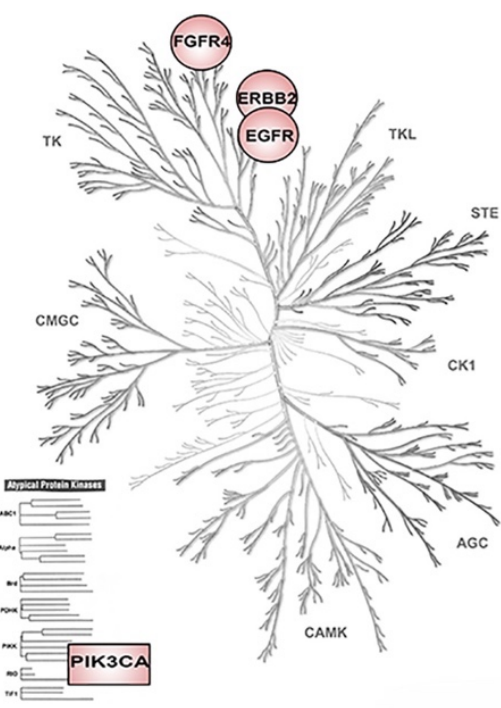

Basal-like / Claudin-low

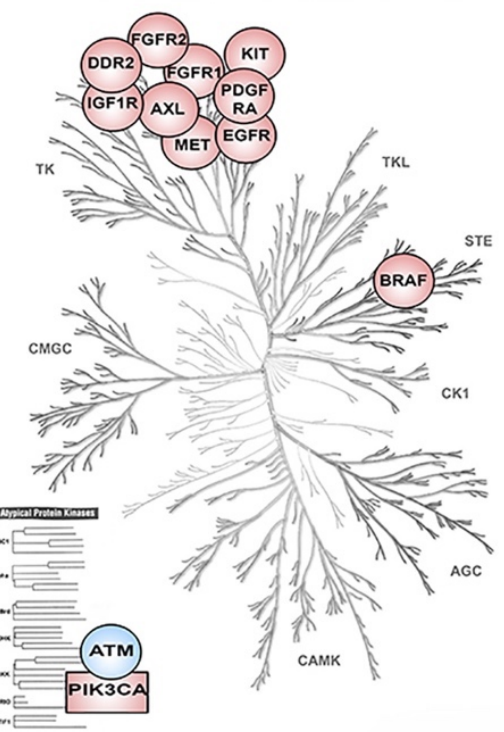

$\overbrace{\text { Amplification }}^{\text {Over Expression }} \bigcirc \begin{gathered}\text { Underexpression } / \\ \text { Deletion }\end{gathered}$ Activating Mutation $\square$ Inactivating Mutation

B.

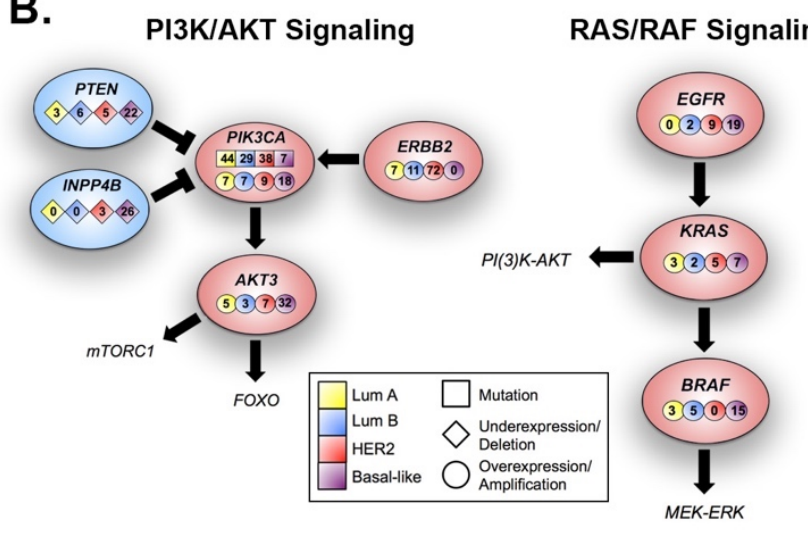

JNK Signaling

Figure 1. Overview of the genomic landscape across breast cancer molecular subtypes. (A) Kinome trees highlighting mutations and copy number alterations found in each subtype of breast cancer. (B) Mutation frequencies in kinase-driven signaling pathways across breast cancer subtypes. Mutation frequencies for each molecular subtype were calculated using the whole genome sequencing data in the TCGA study following PAM50 classification available via cBio Portal (Cancer Genome Atlas Network, 2012; Cerami et al., 2012). Frequencies of mRNA overexpression in breast cancer molecular subtypes were calculated using a two-fold expression z-score threshold on the mRNA expression results of the TCGA study. 
where increased phosphorylation of these biomarkers is observed (Cheng et al., 2015). Luminal A tumors also harbor inactivating mutations of MAP3K1 (13\%) and MAP2K4 (7\%), which regulate JNK and p38 activation (Figure 1B). A hypothesis for the functional role of MAP3K1 and MAP2K4 inactivating mutations in luminal A tumors is their role during mammary gland involution, which involves postlactational apoptosis and tissue remodeling (Avivar-Valderas et al., 2014). Delay or failure of involution is a breast cancer risk factor, possibly because failure of involution could allow cancer progenitor cells to survive.

Luminal B tumors exhibit a higher diversity of mutations than the Luminal A molecular subtype, which occur at lower frequency, with TP53 and PIK3CA being the most frequent at $29 \%$. In addition, the majority of luminal B tumors are clinically characterized by expression of the ER (82\%), but a subset of tumors $(11 \%)$ also express the HER2 receptor. Pathway analysis of Luminal B tumors revealed that proliferation is driven by the transcriptional activity of MYC and FOXM1. Copy number analysis of luminal B tumors confirms amplifications of $C C N D 1, C D K 4$, and $C D K 6$, which suggests dysregulation of cell cycle progression in G1. Amplifications were also found in several members of the FGFR receptor family and IGFR1. Mutually exclusive inactivating mutations were also observed in MAP3K1 (5\%) and MAP2K4 (2\%), at a lower frequency compared to luminal A tumors (Figure 1B).

\section{HER2-enriched Breast Cancer}

The HER2 molecular subtype has a high frequency of HER2-amplification (72\%) and PIK3CA mutation (38\%), but few other kinase and non-kinase genes are significantly mutated in this subtype (Figure 1A and B). Gene expression analysis of HER2 tumors revealed elevated expression of FGFR4, EGFR, and HER2, as well as several other genes in the HER2 amplicon. Copy number analysis of HER2 tumors revealed frequent loss of PTEN and INPP4B, contributing to activation of PI3K-AKT-mTOR signaling in HER2-enriched tumors. Amplification of members of the FGFR receptor family, EGFR, CDK4 and cyclin D1 also highlight several potentially targetable kinases. Reverse Phase Protein Array (RPPA) analysis revealed increased phosphorylation of HER2 and EGFR, in addition to elevated phosphorylation of SRC and ribosomal protein S6, indicating that these pathways drive activation of AKT. 


\section{Basal-like Breast Cancer}

A large subset of basal-like breast cancer is characterized by TP53 mutation or loss (78\%), but this subtype is otherwise devoid of significant somatic mutations, with the only other frequent mutation being PIK3CA (7\%) (Figure 1A and B). Gene expression analyses found the basal-like subtype is characterized by an elevated cell proliferation signature, as well as the expression of basal keratins 5, 6, and 17. Pathway analysis of basal-like breast cancer identified hyper-activated MYC and HIF1 $\alpha$ as key transcription factors controlling the basal-like phenotype. Copy number analysis indicates activation of PI3K signaling through deletion of PTEN and INPP4B. Elevated expression is observed for PIK3CA (18\%), KRAS (7\%), BRAF (15\%) and EGFR (19\%). Several RTKs are also amplified in basal-like tumors, including FGFR1, FGFR2, IGF1R, KIT, MET and PDFGRA. The high proliferation signature in basal-like breast cancer suggests that these tumors are highly responsive to anti-mitotic chemotherapy, but a lack of dominating therapeutic targets and high tumor diversity makes basal-like breast cancer difficult to treat.

\section{Claudin-low Breast Cancer}

While claudin-low tumors are similar to basal-like tumors in being triple negative for ER, PR and HER2, the claudin-low molecular subtype lacks the proliferation gene signature characteristic of basal-like breast cancers. Furthermore, immunohistochemical analysis of claudin-low tumors revealed depletion of cell-cell adhesion genes claudin-3 and E-cadherin relative to other molecular subtypes indicating that these cells exhibit a mesenchymal phenotype (Prat et al., 2010). Claudin-low cells also have elevated expression of genes characteristic of mesenchymal cells including AXL and ZEB1. Claudin-low tumors are also enriched in genes involved in sensing and regulating the extracellular matrix, including the collagen receptor DDR2, and integrin alpha 5.

\section{Kinase Inhibitors Targeting Breast Cancer Molecular Subtypes}

Despite FDA approval of targeted therapies for the luminal and HER2-amplfied subtypes, breast cancer remains a leading cause of cancer-related mortality among women. Expression of the ER and PR in luminal $A$ and $B$ subtypes of breast cancer provide targets for hormone therapies including tamoxifen, which blocks the ability of estrogen to interact with its receptor, and aromatase inhibitors which block the production of estrogen. The HER2 receptor can be targeted in HER2-enriched breast cancer using the 
monoclonal antibodies trastuzumab or pertuzumab, or the small molecule inhibitor lapatinib, which block activation of HER2 and other ERBB family RTKs. However, the majority of basal-like and claudin-low tumors are pathologically classified as triple negative breast cancer (TNBC) and do not express the ER, PR or HER2. Given their negative receptor status, there are currently no FDA-approved targeted therapies for TNBC. However, several kinase-driven growth promoting signaling pathways are dysregulated in TNBC including the RAF-MEK-ERK signaling cascade (Figure 1B), offering potential new targets for the treatment of TNBC. A complete list of kinase inhibitors that are FDA-approved or currently in clinical trials can be found on the following page in Table 1.

\section{HER2 targeted therapies}

HER2 is an ERBB-family RTK that is commonly overexpressed in tumors in the HER2-enriched molecular subtype. HER2 acts a co-receptor for several ligands resulting in the formation of heterodimers with other ERBB family members, such as HER3 and EGFR to stimulate PIK3CA and MAP Kinase pathway activation. Trastuzumab is a humanized monoclonal antibody that binds to the extracellular domain of HER2, preventing ligand-independent activation of the HER2 receptor and eliciting an immune response that results in antibody-dependent cytotoxicity in HER2-positive tumor cells. Early clinical trials of trastuzumab in combination with chemotherapy reported increased time to disease progression (7.4 vs 4.6 months) and increased rate of objective response ( $50 \%$ vs $32 \%$ ) compared to the control arm of the study (Slamon et al., 2009). A four year follow up across multiple studies further illustrated the efficacy of trastuzumab treatment in HER2-amplfied patients with a 39\% reduction in death rate (Perez et al., 2011). As a result, trastuzumab has become the standard of care for women with HER2-enriched breast cancer. Sequencing studies have demonstrated that EGFR, a co-activator of HER2, is often overexpressed or amplified in HER2-enriched breast cancer. Therefore, combining trastuzumab with EGFR inhibitors may have a clinical benefit for HER2 patients with EGFR overexpression. Indeed, recent clinical studies have determined that co-treatment with trastuzumab and the dual EGFR/HER2 inhibitor lapatinib enhances response in patients (Blackwell et al., 2012). Preclinical studies have demonstrated that HER3 is a potent co-activator of HER2 signaling, which can stimulate activation of MAP kinase and PIK3CA pathways. Pertuzumab is a humanized monoclonal antibody that blocks receptor dimerization and inhibits 


\begin{tabular}{|c|c|c|c|}
\hline Family & Target & Drug & Type \\
\hline \multirow[t]{2}{*}{ Ras-MAPK Pathway } & Raf & $\begin{array}{c}\text { Tipifarnib (R115777) } \\
\text { Sorafenib (Bay 43-9006) }\end{array}$ & $\begin{array}{c}\text { farnesytranferase inhibitor } \\
\text { small molecule inhibitor }\end{array}$ \\
\hline & MEK1/2 & $\begin{array}{c}\text { Trametinib (GSK0020212) } \\
\text { Selumetinib (AZD6244) } \\
\text { Binimetinib (MEK162) }\end{array}$ & $\begin{array}{l}\text { small molecule inhibitor } \\
\text { small molecule inhibitor } \\
\text { small molecule inhibitor }\end{array}$ \\
\hline \multirow[t]{3}{*}{ PI3K-AKT-mTOR Pathway } & PI3K & $\begin{array}{c}\text { Buparlisib (BKM120) } \\
\text { BEZ235 } \\
\text { Alpelisib BYL-719 }\end{array}$ & $\begin{array}{l}\text { small molecule inhibitor } \\
\text { small molecule inhibitor } \\
\text { small molecule inhibitor }\end{array}$ \\
\hline & AKT & $\begin{array}{c}\text { MK2206 } \\
\text { GSK2141795 } \\
\text { Afuresertib (GSK2110183) }\end{array}$ & $\begin{array}{l}\text { small molecule inhbitor } \\
\text { small molecule inhibitor } \\
\text { small molecule inhibitor }\end{array}$ \\
\hline & mTOR & $\begin{array}{c}\text { Everolimus } \\
\text { BEZ235 } \\
\text { Temsirolimus (CCI-779) }\end{array}$ & $\begin{array}{l}\text { small molecule inhibitor } \\
\text { small molecule inhibitor } \\
\text { small molecule inhibitor }\end{array}$ \\
\hline \multirow[t]{11}{*}{ Tyrosine Kinases } & $\begin{array}{l}\text { HER2/EGFR } \\
\text { HER2 }\end{array}$ & $\begin{array}{c}\text { Lapatinib (Tykerb) } \\
\text { Trastuzumab (Herceptin) } \\
\text { Pertuzumab (Perjeta) } \\
\text { Neratinib }\end{array}$ & $\begin{array}{l}\text { small molecule inhibitor } \\
\text { monoclonal antibody } \\
\text { monoclonal antibody } \\
\text { small molecule inhibitor }\end{array}$ \\
\hline & EGFR & $\begin{array}{c}\text { Gefitinib } \\
\text { Erlotinib (Tarceva) }\end{array}$ & $\begin{array}{l}\text { small molecule inhibitor } \\
\text { small molecule inhibitor }\end{array}$ \\
\hline & IGF1-R/IR & $\begin{array}{c}\text { BMS-754807 } \\
\text { Cixutumumab (IMC-A12) } \\
\text { Teprotumumab (RV001) } \\
\text { OSI-960 }\end{array}$ & $\begin{array}{l}\text { small molecule inhibitor } \\
\text { monoclonal antibody } \\
\text { monoclonal antibody } \\
\text { small molecule inhibitor }\end{array}$ \\
\hline & FGFR & $\begin{array}{l}\text { Dovitinib (TKI-258) } \\
\text { AZD4547 }\end{array}$ & small molecule inhibitor \\
\hline & VEGFR & $\begin{array}{l}\text { Bevacizumab } \\
\text { Ramucirumab }\end{array}$ & $\begin{array}{l}\text { monoclonal antibody } \\
\text { monoclonal antibody }\end{array}$ \\
\hline & $\begin{array}{c}\text { VEGFR, PDGFR, } \\
\text { FGFR }\end{array}$ & Lucitanib & small molecule inhibitor \\
\hline & $\begin{array}{c}\text { VEGFR, PDGFR, } \\
\text { c-kit, flt3 }\end{array}$ & Sunitinib (SU11248) & small molecule inhibitor \\
\hline & $\begin{array}{l}\text { VEGFR2/3, } \\
\text { PDGFR, c-kit, } \\
\text { p38, flt3 }\end{array}$ & Sorafenib (Bay 43-9006) & small moelcule inhibitor \\
\hline & c-MET & $\begin{array}{l}\text { Tivantinib (ARQ-197) } \\
\text { INC280 (Capmatinib) }\end{array}$ & $\begin{array}{l}\text { small moelcule inhibitor } \\
\text { small moelcule inhibitor }\end{array}$ \\
\hline & c-MET/NEGFR2 & Cabozantinib (XL-184) & small moelcule inhibitor \\
\hline & $\begin{array}{l}\text { SRC-family } \\
\text { kinases }\end{array}$ & Dasatinib & small moelcule inhibitor \\
\hline \multirow[t]{2}{*}{ Cyclin Dependent Kinases } & CDK4/6 & $\begin{array}{c}\text { Palbociclib (PD-0332991) } \\
\text { LEE-011 } \\
\text { Abemaciclib }\end{array}$ & $\begin{array}{l}\text { small molecule inhibitor } \\
\text { small molecule inhibitor } \\
\text { small molecule inhibitor }\end{array}$ \\
\hline & CyclinD1/CDK4 & Ribociclib & small molecule inhibitor \\
\hline
\end{tabular}

Table 1. Targeted Kinase Inhibitors for the Treatment of Breast Cancer (FDA-Approved or in Clinical Trials). 
transactivation of HER2 and HER3 receptors (Capelan et al., 2013). Notably, dimerization of HER2 with HER3 has been published as a major mechanism of resistance to HER2 kinase inhibitors, (N. Dey et al., 2015) and a multicenter clinical trial established significant benefit adding pertuzumab to trastuzumab in patients whose disease had progressed during prior trastuzumab treatment (Baselga et al., 2010). Neratinib is an additional targeted inhibitor that irreversibly binds to multiple members of the HER family including EGFR, HER2 and HER4. Recent results of the ExteNET trial demonstrate that neratinib can be used to extend adjuvant treatment of HER2-positive patients previously treated with trastuzumab (A. Chan et al., 2016). Additionally, Puma Biotechnology has reported a significant increase in disease free survival with a $33 \%$ reduced risk for invasive disease recurrence with neratinib treatment compared to placebo.

\section{PI3K/mTOR pathway inhibitors}

Components of the phosphatidylinositide 3-kinase/mammalian target of rapamycin (PI3K/mTOR) pathway are also commonly dysregulated in multiple subtypes of breast cancer (Figure 1B) making AKT, $\mathrm{PI} 3 \mathrm{~K}$, and mTOR promising kinase targets for treatment. Upon RTK activation, the p85 regulatory subunit of PI3K binds to the phosphorylated RTK, and recruits the p110 catalytic subunit to the cell membrane, where it phosphorylates phosphatidylinositol 4,5 bisphosphate $\left(\mathrm{PIP}_{2}\right)$ at the 3' position. Phosphorylation of $\mathrm{PIP}_{2}$ recruits $\mathrm{PDK} 1$ to the membrane, where it phosphorylates and activates AKT to stimulate proliferation and survival. Activating mutations in PIK3CA or deletion of the PI3K antagonists PTEN and INPP4B phosphatases increases AKT activation, which results in cell cycle progression and inhibition of proapoptotic $\mathrm{Bcl}-2$ family proteins. Therefore, targeting the PI3K signaling pathway to prevent survival of resistant tumors may sensitize patients that were previously unresponsive to therapy. The BOLERO-2 clinical trial found that treatment using the mTOR inhibitor everolimus in combination with an aromatase inhibitor improved progression free survival by more than two fold in hormone receptor-positive, HER2negative patients otherwise refractory to aromatase inhibitor therapy (Piccart et al., 2014). The phase II TAMRAD study similarly determined that patients resistant to endocrine therapies were more sensitive to combination treatment with everolimus (Bachelot et al., 2012). Given these results, the combination of everolimus and aromatase inhibitors has been approved for treatment of advanced stage, ERpositive/PR-positive/HER2-negative patients. HER2-enriched breast cancers also have a high frequency 
of activating mutations in PIK3CA (38\%), and studies have shown that PIK3CA mutations correlate with significantly lower complete response rates in HER2-enriched patients treated with trastuzumab (Loibl et al., 2014). These results suggest that targeting PI3K signaling in HER2-enriched patients may improve response of tumors with mutant PI3KCA. The BOLERO-3 trial found that the addition of everolimus to trastuzumab significantly prolonged progression free survival in patients otherwise insensitive to trastuzumab (Andre et al., 2014). Similar clinical results have been found for treatment of HER2-amplfied, ER-positive and PR-positive patients with the mTOR inhibitor temsirolimus. Several phase II and phase III clinical trials found that the addition of temsirolimus to endocrine or HER2 targeted therapies only benefited heavily pretreated women and had little effect on cohorts of women naïve to treatment (S. Chan et al., 2005; Fleming et al., 2012; Wolff et al., 2013). Everolimus is also being tested in triple negative patients (NCT01939418). Additionally, inhibitors targeting downstream effectors of the pathway including AKT (MK2206 and GSK2141795) and PI3K (BKM120) are currently in clinical trials as single agents and in combination with other currently approved drugs in all subtypes of breast cancer.

\section{MAPK pathway inhibitors}

The mitogen activated protein kinase (MAPK) pathway plays an essential role in normal cell proliferation and differentiation, and dysregulation of this pathway has been shown to contribute to many different kinds of cancer. Members of the MAPK pathway are also aberrantly expressed and mutated in breast cancer. Triple negative breast cancer patients in particular show overexpression of members of the MAPK pathway including KRAS and BRAF (Figure 1B). Chemotherapy remains the only approved therapy for TNBC patients due to the absence of ER, PR and HER2 expression. It is therefore important to develop targeted therapies for TNBC. Overexpression of KRAS and BRAF in TNBC may stimulate MAPK pathway activation, which can be targeted by inhibition of downstream effector kinases. There are several ongoing clinical trials studying the benefit of treating advanced TNBC patients with MEK inhibitors including trametinib, selumetinib and binimetinib. Preclinical studies have also suggested that combined inhibition of the MAPK and PI3K/mTOR pathways could be beneficial to breast cancer patients by preventing adaptive resistance mechanisms to single inhibitor therapy (Saini et al., 2013). Trials have assessed the safety of combined MEK inhibition with PI3K or AKT inhibitors in multiple types of cancer 
(NCT01138085, NCT01476137, NCT01562275, NCT01363232) and clinical trials are ongoing to test the efficacy of this combination therapy in advanced breast cancer (NCT01390818).

Receptor Tyrosine Kinase Inhibitors

There are several other RTKs that have been identified as drivers of disease progression in breast cancer subtypes. These RTKs also have the potential to activate alternative signaling pathways to drive resistance to single inhibitor treatments. Clinical trials are ongoing to assess treatment of HER2enriched tumors with the IGF-1R/IR inhibitor BMS-754807 (NCT00788333). The IGF-1R inhibitor cixutumumab was recently evaluated in a phase II clinical trial in combination with endocrine therapy (Gradishar et al., 2016). The results of this study showed little additive effect of combination cixutumumab treatment with endocrine therapy and IGF1-R expression did not correlate with patient response to therapy. However, mRNA analysis revealed that low expression of total insulin receptor resulted in improved progression free survival regardless of treatment. Cixutumumab has also been evaluated in combination with the targeted mTOR inhibitor temsirolimus in a phase I clinical study (Ma et al., 2013), and the efficacy of combination cixutumumab and temsirolimus treatment in locally recurrent or metastatic breast cancer is ongoing in a phase II clinical trial (NCT00699491).

Preclinical studies have defined additional kinase targets amplified or activated in triple negative breast cancers including EGFR, VEGFR, PDGFR and SRC family kinases. EGFR is overexpressed in $19 \%$ of basal-like breast cancer, which suggests that these tumors may be sensitive to EGFR inhibitors, but initial clinical trials with EGFR inhibitors have been disappointing. One potential explanation for the failure of EGFR inhibition in clinical trials is a lack of criterion for identifying EGFR amplification in patients. Additionally, clinical trials have evaluated combining multiple RTK inhibitors in metastatic breast cancer patients. A phase II trial found little additive effect of combining the VEGFR inhibitor bevacizumab with erlotinib, but this study was performed in unselected patients (Dickler et al., 2008). These studies highlight the need for biomarker analysis in future clinical studies in order to identify breast cancer patients likely to respond to targeted inhibition of RTKs.

Several kinase inhibitors of the VEGF receptor have been studied in treating multiple breast cancer subtypes. VEGFR is an essential regulator of angiogenesis, which is necessary to support tumor growth. Angiogenesis is also necessary for the progression of metastasis making VEGF receptors 
potential therapeutic targets. Several clinical trials have investigated the efficacy of adding the monoclonal anti-VEGFR antibody bevacizumab to standard chemotherapy in hormone receptor negative patients (Cobleigh et al., 2003; Lang et al., 2012; K. D. Miller et al., 2005; Robert et al., 2011). The results of these studies have been varied with several initial clinical trials showing significantly improved progression free survival in TNBC patients treated with bevacizumab. More recent trials have failed to show improved survival in estrogen receptor negative cohorts and the FDA approval of bevacizumab in breast cancer patients was ultimately withdrawn due to lack of efficacy with increased toxicity of treatment. Several other kinases inhibitors targeting VEGF receptors in TNBC are still being evaluated in clinical trials, including the broad-acting inhibitors sunitinib (NCT00887575) and sorafenib (NCT00825734, NCT00544167).

Despite the initial promise of kinase inhibitors for the treatment of many cancers, these therapies often fail due the emergence of drug resistance after a pathological partial response. Recent studies have shown that this resistance can be caused by epigenetic remodeling that enhances the transcription of genes to stimulate pathway reactivation in the presence of the drug, or activate parallel signaling pathways that promote survival. Chapter 2 will briefly overview the genetic and epigenetic factors that regulate transcription in human cells, and how these mechanisms can become dysregulated in cancer. 


\section{CHAPTER 2: HUMAN TRANSCRIPTIONAL REGULATION IN CANCER}

The human genome contains the genetic information for all cells, and it is simply the interpretation and expression of the genome that determines cell identity. Therefore, gene expression is highly regulated during development to control differentiation and fate. This requires multiple layers of regulation that can activate or repress transcription either temporarily or permanently. This chapter will briefly overview the regulatory elements involved in human transcriptional regulation in normal development and oncogenesis.

\section{DNA Regulatory Elements in Mammalian Transcription}

There are two regulatory elements encoded in the DNA: a core promoter region that recruits RNA Polymerase II (RNAPII) to the transcription start site (TSS), and an enhancer region that recruits transcriptional activators (Figure 2). The core promoter is an approximately 100 bp region surrounding the TSS at the 5' end of the target gene, which typically contains a TATA-box binding sequence $<$ TATAa/tAa/t> positioned -25 to -30 bp from the TSS, and a pyrimidine-rich initiator sequence <YYANt/aYY> near the TSS (Javahery et al., 1994; Nakajima et al., 1988). These sequences recruit the pre-initiation complex (PIC), and determine the orientation and position of transcriptional initiation (Roeder, 1996). However, core promoter regions demonstrate low basal transcriptional activity and are often insufficient to reach maximal transcriptional output from the target gene. Novel sequencing techniques, along with advances in computational biology have demonstrated that distal enhancer regions amplify the transcriptional activity of core promoter regions (Stadhouders et al., 2012). Enhancers are large regulatory regions that bind transcription factors and interact with subunits of the Mediator complex to help recruit RNAPII to the target gene and increase transcriptional output. Enhancers are several hundred kilobases in length, and typically found in intergenic regions. These regions cooperate with promoters to regulate gene expression during differentiation and development to control cell fate and identity by pairing enhancers activated during a specific developmental state with its cognate promoter. 


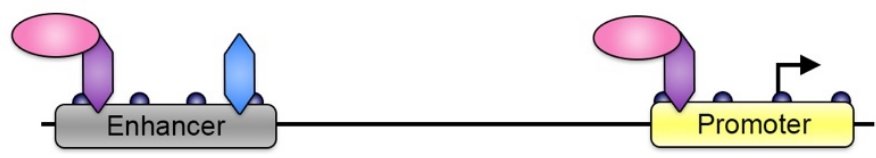

2

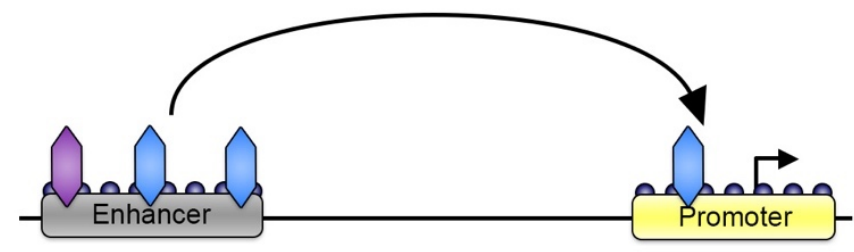

3
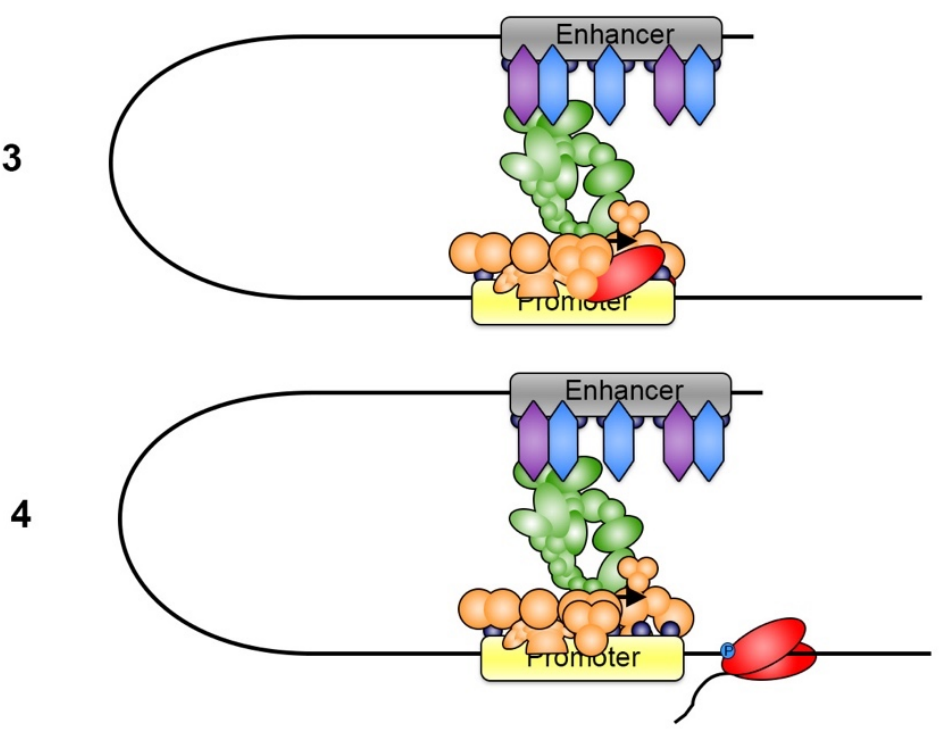

Legend

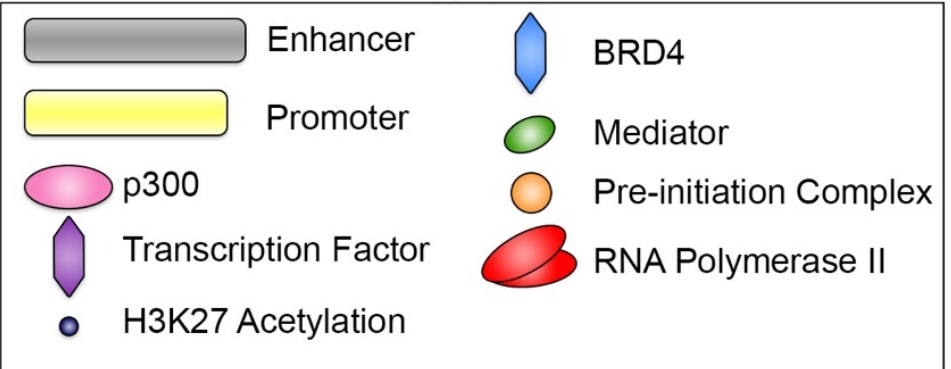

Figure 2: Enhancer Regulation of Mammalian Transcription ${ }^{1}$. (1) Transcription factors and transcriptional co-activators, bind the promoter and enhancer regions of the target gene, and recruit histone acetyltransferase p300. (2) Increased H3K27ac recruits additional transcriptional co-activators such as BRD4 to promoter and enhancer region, DNA loops to bring enhancer proximal to the target gene promoter. (3) Mediator complex binds transcriptional co-activators, and the PIC and RNAPII bind the promoter region, but RNAPII remains paused at the TSS. (4) PIC phosphorylates the CTD of RNAPII, which releases RNAPII to transcribe the target gene.

${ }^{1}$ Illustration adapted from (Soutourina, 2018) 


\section{Role Chromatin Structure in the Regulation of Gene Expression}

The transcriptional activity of promoter and enhancer regions is tightly regulated by chromatin structure and histone modifications that are established early in development. Nucleosome packing and higher-order chromatin structures play essential roles in the regulation of gene expression and cell identity. Genome-wide assessment of chromatin structure identified an inverse correlation between nucleosome occupancy and transcriptional activity, indicating that DNA is most accessible in regions of active transcription (C.-K. Lee et al., 2004). Additionally, the regulation of chromatin structure plays a key role in development and the maintenance of differentiation state. Studies of the regulation of chromatin structures in embryonic stem cells revealed that these cells have relaxed and permissive euchromatin that is maintained by the chromatin remodeling factor CHD1 (Gaspar-Maia et al., 2009). Depletion of CHD1 results in the accumulation of heterochromatin and loss of pluripotency, suggesting that the maintenance of euchromatin is essential in embryonic stem cells. Furthermore, a survey of six different human cell types revealed a unique chromatin fingerprint for each cell type, indicating that chromatin structure plays an essential role in the maintenance of identity (Ernst and Kellis, 2013).

Higher-order chromatin structure regulates gene expression by controlling the proximity of enhancers and core promoter regions in specific cell types. Chromatin looping helps maintain specificity of active enhancer regions for their cognate promoter and insulate the target genes from improper interactions (Hnisz et al., 2016). Analysis of chromatin structure using chromatin conformation capture demonstrates that chromatin is partitioned into topological association domains (TADs) that conformationally restrict the binding of promoters and enhancer regions (Zabidi and Stark, 2016). These TADs are observed in all cell types and evolutionarily conserved, and disruption of these domains leads dysregulation of gene expression, suggesting this a fundamental method of transcriptional regulation. The boundaries of these domains are defined by insulator proteins such as CCCTC-binding factor (CTCF), which stabilize loop formation and control the propagation of histone marks. CTCF recruits cohesin, which binds the ends of the chromatin loop to stabilize the TAD. Depletion of either CTCF or cohesin disrupts chromatin loop formation and results in gene dysregulation, indicating that the CTCF and cohesin are essential to the formation and maintenance of chromatin looping (Hansen et al., 2017). 


\section{Role of DNA Methylation in Transcriptional Regulation}

DNA methylation is a heritable epigenetic modification of cytosine-guanine dinucleotides $(\mathrm{CpG})$ that is enriched in heterochromatin and observed in the promoter region of transcriptionally silenced genes (Jones and Takai, 2001). CpG dinucleotides are distributed throughout the genome, but highly enriched in regions called CpG islands, which are found in the promoter region of $60-70 \%$ of human genes. DNA methyltransferases (DNMTs) catalyze the conversion of cytosine to 5-methyl cytosine by transferring a methyl group from the donor S-adenosyl-L-methionine to the $5^{\text {th }}$ carbon of the cytosine base. DNMT1 is considered a maintenance methyltransferase because it has high affinity for hemimethylated DNA strands, and catalyzes the methylation of cytosine during DNA replication (Biswas and Rao, 2017). In contrast, DNMT3A and DMNT3B have higher affinity for unmethylated DNA, and deposit de novo methylation at $\mathrm{CpG}$ islands. Methylation of $\mathrm{CpG}$ islands recruits methyl binding proteins that can exclude transcriptional activators and silence transcription.

\section{Role of Histone Modifications in Transcriptional Regulation}

The $\mathrm{N}$-terminal tails of histones can be post-translationally modified to regulate chromatin structure and transcriptional activity of the target locus. There are three general classes of histone modifications: methylation, acetylation, and phosphorylation. The position of each of these modifications on a specific histone isoform creates a code that can be interpreted by histone binding proteins to activate or repress transcription. The histone code is incredibly complex, with each histone having unique modifications that regulate transcription at specific developmental stages or at certain regions genome. Therefore, for simplicity, l'll review the most well studied histone modifications that regulate transcription in normal and tumorigenic cells. Methylation of the $\mathrm{N}$-terminal tail of histone $\mathrm{H} 3$ at lysine $4(\mathrm{H} 3 \mathrm{~K} 4)$ is catalyzed by histone methyltransferases (HMTs) at enhancers and core promoter regions associated with poised or actively transcribed genes. H3K4 can be methylated by a family of SET domain containing HMTs including SET1A, SET1B, MLL1, MLL2, MLL3, MLL4, SMYD1, SMYD2, SET7/9 and PRDM9 (Hyun et al., 2017). Crystal structures of SET domain family members demonstrate that the SET domain catalyzes methyl transfer by binding the histone substrate and stabilizing the methyl donor S-adenosyl-Lmethionine (Dillon et al., 2005). Methylation of histones is a reversible modification, which allows the cell 
to finely tune the histone code and differentially regulate gene expression during development. Methylation at H3K4 can be removed by the lysine demethylases KDM1A, KDM1B, KDM5A, KDM5B, KDM5C, KDM5D, and RIOX1 (Hyun et al., 2017). It remains unclear, however, the mechanism by which histone methyltransferases and demethylases recognize specific genomic loci for catalysis. ChIP-Seq for $\mathrm{H} 3 \mathrm{~K} 4$ methylation identified an enrichment for $\mathrm{H} 3 \mathrm{~K} 4$ monomethylation (H3K4me1) at enhancer regions associated with active or poised gene transcription, whereas $\mathrm{H} 3 \mathrm{~K} 4$ trimethylation $(\mathrm{H} 3 \mathrm{~K} 4 \mathrm{me} 3)$ is a hallmark of the promoter region of actively transcribed or poised genes (Heintzman et al., 2009). Histone trimethylation of histone $\mathrm{H} 3$ also occurs at lysine 27 (H3K27me3), which is transferred by the SET domain methyltransferases EZH1 and EZH2. H3K27me3 is primarily a repressive histone modification, which is observed in promoter regions associated with transcriptionally silenced genes and enriched in methylated CpG islands. However, H3K27me3 is also observed with H3K4me1 at enhancer and promoter regions of genes poised for transcription. These bivalent promoter regions are essential to the regulation of differentiation because they allow the target gene to remain poised for activation but transcriptionally silent in the absence of a differentiation signal (Creyghton et al., 2010). Gene silencing by H3K27me3 is antagonized by the transfer of an acetyl group from acetyl-CoA to histone H3 lysine 27 (H3K27ac) by the histone acetyltransferases CBP and p300 (Tie et al., 2009). Acetylation at H3K27 is reversible and can be removed by a family of enzymes called histone deacetylases (HDACs). ChIP-Seq analysis of H3K27ac identified enrichment at enhancers and core promoter regions of actively transcribed genes, and was highly correlated with p300 binding. Chromatin accessibility assays following acetylation of H3K27 indicate that acetylation induces chromatin relaxation and a permissive chromatin state that increases binding of transcriptional co-activators such as the histone acetylation reader BRD4 (Mueller et al., 2017). These results indicate that $\mathrm{p} 300$ binding increases $\mathrm{H} 3 \mathrm{~K} 27 \mathrm{ac}$ at enhancers and core promoter regions to activate transcription. Furthermore, several studies demonstrate that transcription factors can bind and recruit p300 to acetylate specific DNA sequences in activated enhancer regions (Alver et al., 2017; Rubin et al., 2017; Zabidi et al., 2015). Histone acetylation readers such as BRD4, can bind acetylated histones to recruit Mediator and PIC to enhance transcription at sites of H3K27ac (Jang et al., 2005). BRD4 can also bind transcription factors for locus-specific localization of transcriptional co-activators to enhance transcription of target genes (S.-Y. Wu et al., 2013). In this manner, cells can control histone methylation 
or acetylation to modulate the activity of enhancers and promoters to control transcription during development and maintain cell identity.

\section{Epigenetic Dysregulation in Cancer}

The regulation of chromatin structure and modification is essential to normal development and differentiation, but can become dysregulated during tumorigenesis. While normal development requires tight regulation of chromatin structure and histone marks, these regulatory mechanisms can be used by cancer cells to silence the expression of tumor suppressors and activate the expression of oncogenes. Whole exome sequencing of diverse tumor types identified mutations in the SWI/SNF chromatin remodeling complex in greater than $20 \%$ of cancers (Kadoch and Crabtree, 2015). These mutations can affect either localization or activity, leading to dysregulation of nucleosome positioning and gene transcription. Mutations in the SWI/SNF chromatin remodeling complex prevents nucleosome eviction of H3K27me3 histones at the CDKN2A locus, which silences p16 expression and increases proliferation (Wilson et al., 2010). Additionally, expression CDKN2A can be silenced by DNA methylation, which has been shown to increase at the CDKN2A locus during progression of lung cancer from lung airway basal cell hyperplasia (17\%) to squamous metaplasia (24\%) to carcinoma in situ (50\%) (Belinsky et al., 1998). Genome-wide analysis of DNA methylation identified increased methylation at 5 to $10 \%$ of CpG islands in normally unmethylated promoters. Further analysis of the role of methylation in colon cancer cell proliferation identified focal gains of DNA methylation at $\mathrm{CpG}$ islands in the promoter regions of several genes compared to a DNA methyltransferase knockout control (De Carvalho et al., 2012). Ectopic expression decreased proliferation of colon cancer cells, suggesting that epigenetic silencing of these genes by DNA methylation promotes tumorigenesis in these cells. The dysregulation of histone acetylation is also observed in several cancers. Fusion of the histone acetyltransferase MOZ with the transcription factor TIF2 is sufficient to transform myeloid progenitor cells and induce acute myeloid leukemia in mouse bone marrow transplant models (Deguchi et al., 2003). Furthermore, binding of the histone acetyltransferase p300 to the MOZ-TIF2 gene fusion is necessary for transformation, suggesting that dysregulation of histone acetylation is plays a role in tumorigenesis. 
Chapter 3 will review the mechanisms of resistance to targeted therapy, as well as summarize my work in collaboration with Zawistowski and Bevill et al., studying the mechanism of epigenetic remodeling and the role of transcriptional activation in response to targeted therapy. 


\section{CHAPTER 3: MECHANISMS OF RESISTANCE TO THERAPY}

Beginning with the FDA approval of imatinib for the treatment of chronic myeloid leukemia in 2001, kinase inhibitors have been developed for nearly all oncogenic indications with the promise of potent, highly selective and non-toxic compounds for the treatment of cancer. Imatinib targets the constitutively active BCR-ABL gene fusion of the BCR and ABL kinases caused by a chromosomal translocation in leukemia patients. This drug is incredibly effective as a monotherapy, with a ten-year survival rate of $80 \%$ in CML patients treated with imatinib (Hehlmann et al., 2017). The excitement from this success cannot be understated: patients that were previously hospitalized with stable or progressive disease started taking a once-daily tablet and were able to get out bed or even exercise (Dreifus, 2009). However, in the years since the approval of imatinib, we have come to realize that the success of this drug in CML patients is the exception, not the rule. In many cases, patients treated with a kinase inhibitor will exhibit a pathological partial response, followed by progression during continued treatment. Kinase inhibitor resistance can be classified into three general categories: intrinsic resistance (Figure 3A), acquired resistance, and adaptive resistance.

\section{Intrinsic Resistance}

The Cancer Genome Atlas study sequenced over 500 breast cancer tumors and identified over 30,000 somatic mutations, most of which are believed to be background mutations unrelated to cancer progression. Previous studies suggest that initiation of tumor progression requires genomic alterations in founding tumor cells (Stratton et al., 2009). Deep sequencing and the development of molecular subtypes have improved initial diagnosis, but tumors can carry pre-existing secondary mutations that confer resistance to therapy, leading to non-durable response to the drug (Stratton et al., 2009). A study of HER2-positive breast cancer in a genetically engineered mouse model revealed that mice with a PIK3CA mutation in combination with HER2 amplification demonstrated shorter tumor latencies and were more likely to develop lung metastases (Hanker et al., 2013). Furthermore, these mice were resistant to 


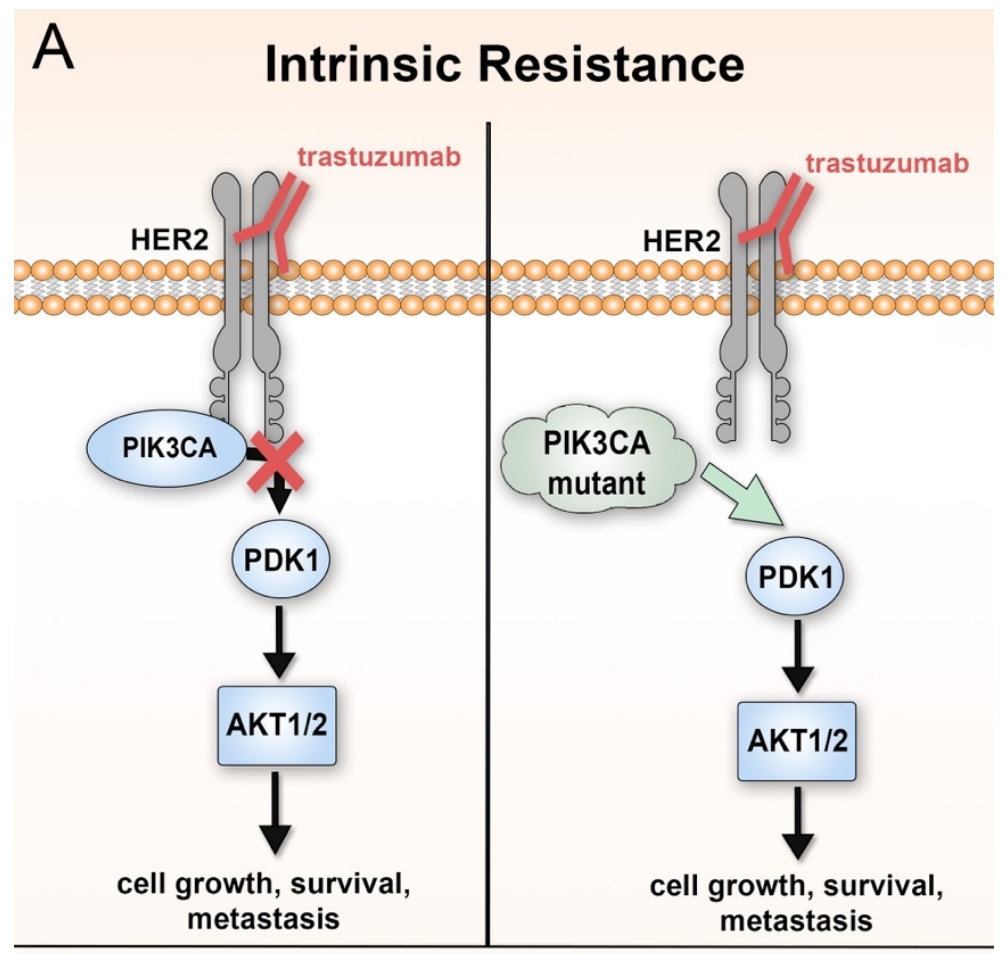

\section{B Acquired Resistance}

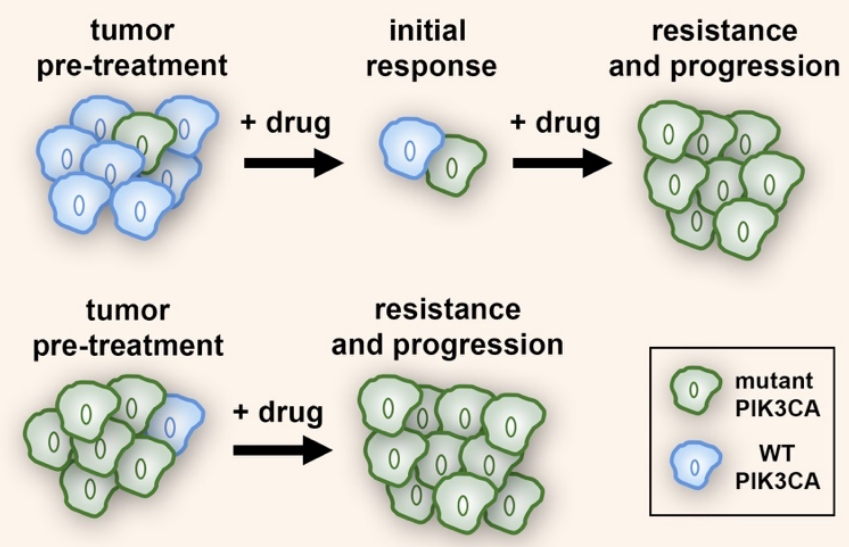

Figure 3. Intrinsic and acquired resistance mechanisms in breast cancer. (A) HER2-enriched breast cancer cells can harbor PIK3CA mutations, which drive intrinsic resistance to targeted HER2 receptor therapy (trastuzumab). During resistance, mutant PIK3CA is able to drive downstream growth signaling through AKT independent of HER2 receptor activity. (B) During acquired resistance, tumors harboring mutations such as PIK3CA are selected for over a time course of treatment resulting in an initial tumor response followed by outgrowth of resistant PIK3CA mutant cells. Tumor populations can also be pre-enriched for a resistance mutant before treatment leading to continued growth upon treatment. 
trastuzumab alone and in combination with either lapatinib or pertuzumab. These data suggested that mutations in PIK3CA reduce oncogene addiction to HER2 and drive intrinsic resistance to HER2 targeted therapies by activating growth signaling independent of the HER2 receptor (Figure 3A). Similarly, FGFR1 amplification is observed in $16-27 \%$ of luminal B breast tumors, and is associated with poor prognosis and resistance to hormone therapy. In a panel of luminal breast cancer cell lines, FGFR1 amplification results in enhanced ligand-independent signaling, and increased activation of the PI3K and MAPK pathways. Furthermore, FGFR1 amplification increased resistance to 4-hydroxytamoxifen, and knockdown of FGFR1 by siRNA re-sensitized cells to hormone therapy. These data demonstrate that amplification of FGFR1 in luminal breast cancer results in increased activation of the PI3K and MAPK signaling pathways, and reduces estrogen dependence in luminal breast tumors.

\section{Acquired Resistance}

Several studies following treatment with targeted inhibitors have demonstrated that genomic alterations during the course of treatment can increase resistance to therapy. Single molecule sequencing of breast tumors demonstrates that point mutations occur at low frequency and accumulate gradually, increasing tumor diversity (Wang et al., 2014). A serial sequencing study of circulating tumor DNA from breast cancer patients before and after treatment demonstrated that low frequency mutations can be enriched during the course of treatment (Murtaza et al., 2013). Deep sequencing of breast cancer patients was used to quantify the enrichment of mutations in circulating tumor DNA before and after treatment with paclitaxel to identify possible mechanisms of resistance to the drug. Deep sequencing demonstrated that mutant PIK3CA in circulating tumor DNA rose from $14 \%$ to $34 \%$ during the course of treatment with paclitaxel. Previous studies have demonstrated that PIK3CA mutations confer resistance to paclitaxel, which suggests that drug-resistant cells are enriched during the course of treatment (Isakoff et al., 2005). These results demonstrate that low frequency mutations that confer a survival benefit can be enriched during the course of treatment, and result in resistance to therapy after an initial therapeutic response (Figure 3B). In luminal breast cancer, researchers sequenced the tumors of 11 relapsed patients following treatment with hormone therapy to identify mechanisms of resistance to the drug (Robinson et al., 2013). Analysis of the sequencing results revealed activating mutations in the ligand- 
binding domain of the ER. These mutations cause activation of the ER independent of ligand, and allow tumor proliferation in the presence of the drug. This study suggests that ligand-independent activation of the ER induces proliferation of cells harboring the mutation and these cells are selected during treatment resulting in increased resistance to hormone therapy in luminal breast cancer.

\section{Adaptive Resistance}

While the incidence of genomic alteration in kinase signaling pathways may suggest several kinases are tractable drug targets, the resiliency and plasticity of these signaling networks remains a challenge for the durability of kinase inhibitors as single agents (Figure 4A). An in vitro study of luminal breast cancer cell lines used RPPA to study kinome activation in response to long-term estrogen deprivation to identify mechanisms of resistance to hormone therapy in luminal breast cancer. These experiments identified increased activation of mTORC1 and PDK1 in response to hormone deprivation, which was abrogated by treatment with the PI3K-mTOR inhibitor BEZ-235 (T. W. Miller et al., 2010).

Targeted therapies have dramatically increased survival of patients with HER2-enriched breast tumors, but recent studies have shown that blocking activation of HER2 by biological or chemical inhibitors induces rapid adaptive responses that overcome the action of the drug (Stuhlmiller et al., 2015). This adaptation can lead to stable resistance that allows the cells to survive and proliferate in the presence of the drug. While there is significant heterogeneity in the response to drug across multiple HER2-positive breast cancer cell lines, inhibition of HER2 by the chemical inhibitor lapatinib generally leads to FOXO-dependent transcriptional upregulation of multiple RTKs that re-activate the MAPK and AKT pathways. Several studies have demonstrated that inhibition of HER2 activity induces expression of HER3 and IGF1R, which can be phosphorylated by residual HER2 kinase activity, leading to pathway reactivation and resistance (Garrett et al., 2011; Huang et al., 2010). Activation of non-receptor tyrosine kinases has been observed upon inhibition of HER2-amplfied breast cancer cells with lapatinib, including multiple SRC family kinase members (Rexer et al., 2011). The transcriptional upregulation of a combination of kinases and the heterogeneity of this response in multiple breast cancer cell lines highlights the plasticity of kinase signaling networks and the difficulty in identifying secondary drug targets 


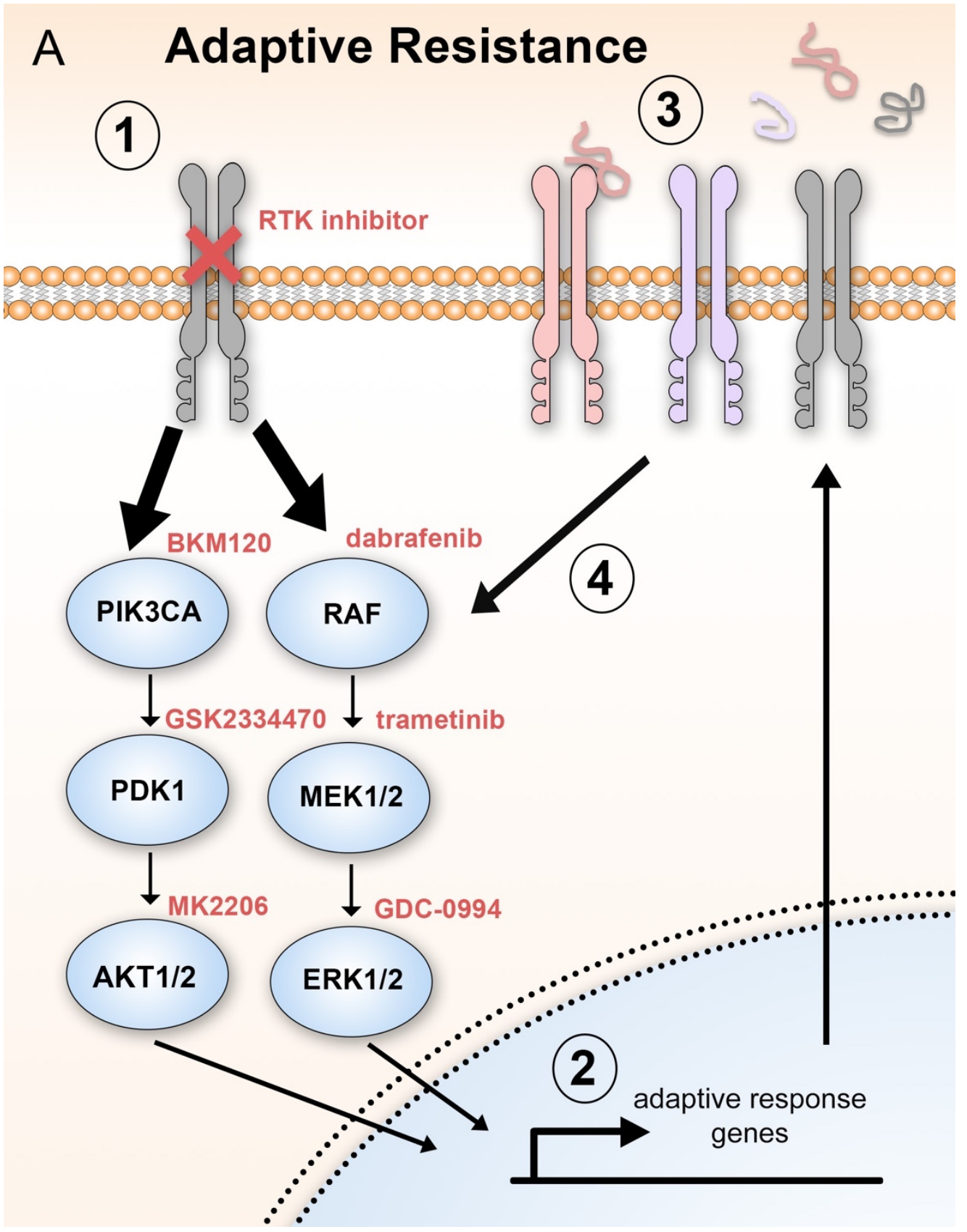

Figure 4: Adaptive resistance mechanisms in breast cancer. (A) (1) Inhibition of target kinase induces (2) loss of negative feedback regulation, which results in a transcriptional response with induction of adaptive response genes and RTKs. (3) RTKs are then transported to the cell membrane where autocrine-paracrine activation of adaptive RTKs drives reactivation of growth signaling (4). 
for combination treatments in HER2-positive breast cancer. The high incidence of PIK3CA amplification in HER2 breast cancer (39\%) suggests that these cancers may be sensitive to therapies targeting the PI3KAKT signaling pathway. However, similar to lapatinib studies, treatment with an AKT inhibitor resulted in activation of HER3, IGF1R, and INSR in as little as 24 hours following treatment (Chandarlapaty et al., 2011). These studies highlight the adaptability of HER2-addicted cells to multiple targeted inhibitor treatments.

Triple negative breast cancers in the basal-like and claudin-low molecular subtypes are characteristically depleted of ER, PR and HER2 expression, and no molecular targeted therapy is currently available for treatment of these molecular subtypes. Aside from PIK3CA mutations, triple negative breast cancers are characterized by a limited number of mutations in genes of druggable targets. The role of gene amplifications in tumor cell proliferation and survival remains unclear, but genes in the PI3K-AKT and MAPK pathways are commonly amplified in basal-like breast cancer, including PIK3CA, KRAS, BRAF, and EGFR. Significant amplification of genes in the MAPK pathway suggests that targeting this pathway may be an effective molecular therapy for this disease. Treatment of triple negative cell lines with the MEK inhibitor selumetinib induced rapid destabilization and degradation of c-Myc, resulting in the upregulation and activation of RTKs including PDGFR $\beta$, DDR1 and VEGFR2 within as little as 24 hours following treatment (Duncan et al., 2012). In each case of adaptive resistance, targeting kinases involved in proliferation and survival signaling led to pathway re-activation by the loss of negative feedback, and transcriptional upregulation of alternative kinases to promote pathway reactivation or bypass signaling in the presence of the drug (Figure 4A).

\section{Subtype Specific Gene Expression in Response to Trametinib in TNBC}

Work published in collaboration with Zawistowski and Bevill et al., demonstrates that triple negative breast cancer cell lines can be classified in basal-like or claudin-low molecular subtypes using RNA-Seq gene expression analysis. Basal-like breast cancers are enriched in epithelial genes including KRT5, KRT17, and ITGB4, whereas claudin-low breast cancers express mesenchymal genes VIM, SNAI1, and VEGFA (Perou et al., 2000; Prat et al., 2010). In response to acute trametinib treatment, the basal-like breast cancer cell line HCC1806 rapidly upregulates receptor tyrosine kinases KIT and 
A

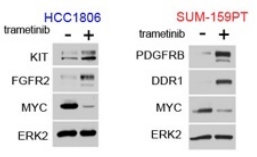

B

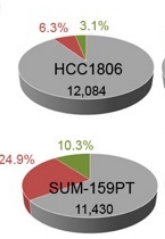

Basal-like
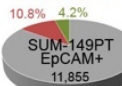
$7.5 \% 5.7 \%$

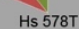

Hs 578T

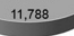

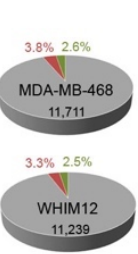

C

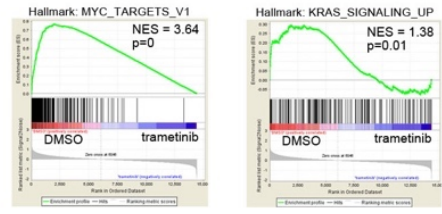

D
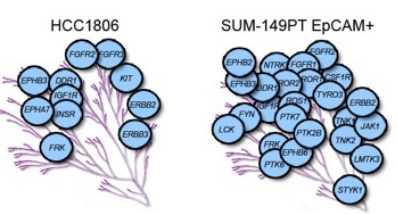

MDA-MB-468

SUM-159PT

Hs 578T

WHIM12
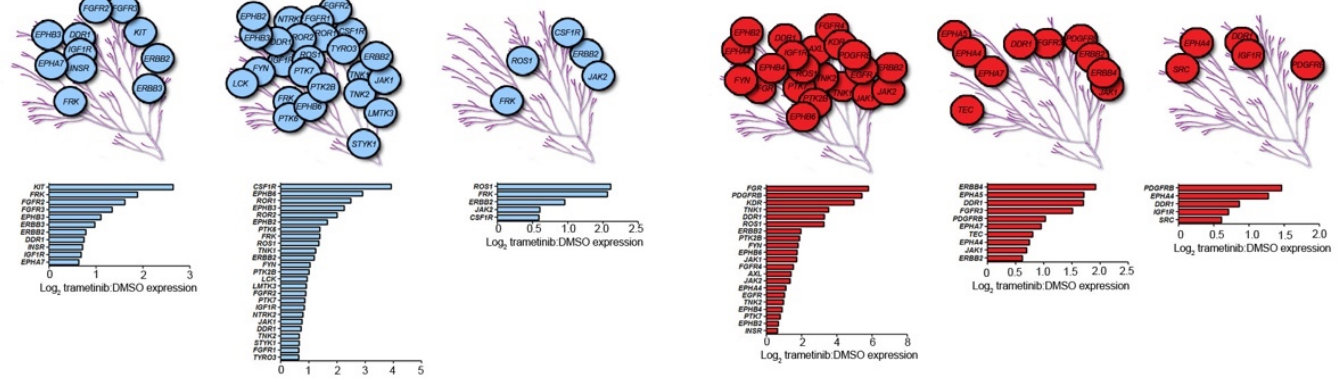

E
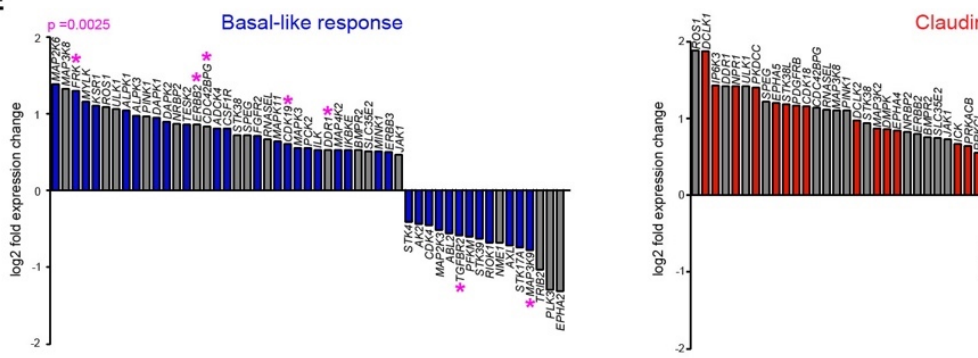

Claudin-low response

$\mathbf{F}$

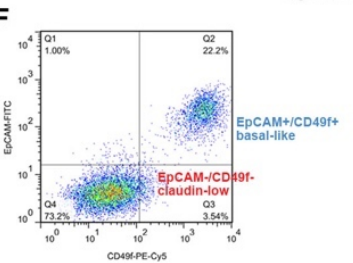

G

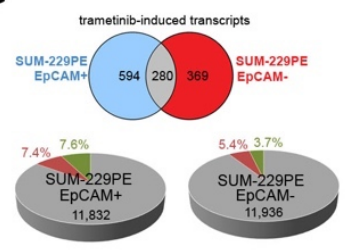

$\mathrm{H}$ trate

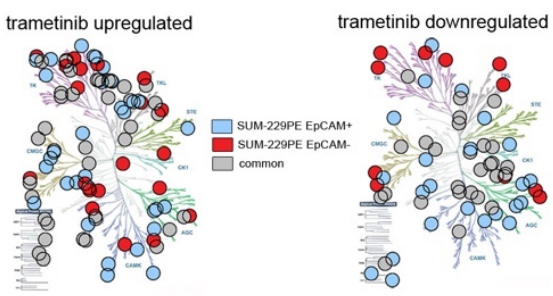

I

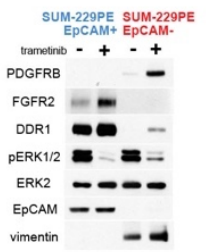

Figure 5: Unique Adaptive Response to Trametinib Treatment in Triple Negative Breast Cancer Subtypes². (A) Immunoblot analysis of RTK expression in distinct molecular subtypes of breast cancer. (B) Transcriptional response to trametinib treatment in basal-like and claudin low cell lines following $24 \mathrm{hr}$ treatment with $500 \mathrm{nM}$ trametinib. (C) GSEA analysis of gene expression in SUM-159 cells following acute trametinib treatment. (D) Tyrosine kinases induced greater than 1.5-fold in basal-like (blue) and claudin-low (red) cell lines in response to trametinib. (E) Significant kinases ( $p$-value $<.05)$ identified by DESeq analysis of the kinome response in basallike and claudin-low breast cancer cell lines. Unique kinases are highlighted in basal (blue) and claudin-low (red), common kinases shown in grey. Kinases identified in patient samples indicated with pink asterisk. (F) Flow cytometry analysis of SUM-229PE cells following staining with epithelial antigens EpCAM and CD49f. (G) Transcriptional response of EpCAM+ and EpCAM- subpopulations following $24 \mathrm{hr}$ treatment with $30 \mathrm{nM}$ trametinib. Venn diagram displays uniquely upregulated genes identified in EpCAM+ (blue) and EpCAM- (red). Commonly upregulated genes highlighted in grey. $(\mathrm{H})$ Kinome response to trametinib treatment distributed across all kinase families in EpCAM+ (blue) EpCAM- (red) or both (grey). (I) Immunoblot analysis of kinase expression in EpCAM+ and EpCAM- isolated subpopulations following $24 \mathrm{hr}$ treatment with $30 \mathrm{nM}$ trametinib.

${ }^{2}$ Figure adapted from (Zawistowski et al., 2017) 
FGFR2, a response which is distinct from the claudin-low cell line SUM-159PT, which upregulates RTKs PDGFR $\beta$ and DDR1 (Figure 5A). RNA sequencing was performed following acute treatment with trametinib of three basal like cell lines HCC1806, SUM-149 EpCAM+, and MDA-MB-468, as well as three claudin-low cell lines SUM-159PT, HS 578T, and WHIM12. Each of these six cell lines dynamically respond to trametinib treatment, with a total transcriptional response ranging from $5.8 \%$ of the transcriptome in WHIM12, to $35.2 \%$ of the transcriptome in SUM-159 cells (Figure 5B). GSEA analysis of the transcriptional response in SUM-159 cells reveals the enrichment of MYC targets in the control treated sample, indicating that loss of MYC observed in immunblots of SUM-159 cells following trametinib treatment (Figure 5A) results in downregulation of MYC target expression. In each of the triple-negative breast cancer cell lines we tested, we observed the upregulation of tyrosine kinases in response to trametinib treatment. Upregulated tyrosine kinases identified in each cell line basal like (blue) and claudinlow (red) cell lines are highlighted on the tyrosine kinase branch of the kinome tree (Figure 5D). We identified tyrosine kinases that are commonly upregulated in both basal-like and claudin-low breast cancer cell lines, highlighted in grey (Figure 5E), which suggests that MYC may bind similar DNA sequences or transcriptional factors at these genes and loss of MYC activates target gene transcription. However, we also observe the upregulation of tyrosine kinases in response to trametinib treatment that are distinct in basal-like (blue) and claudin-low (red) breast cancer cell lines (Figure 5E). This suggests that there are unique regulatory elements in basal-like and claudin-low breast cancer that determine the transcriptional response to trametinib in these molecular subtypes. The SUM-229PE cell line was isolated from the pleural effusion of a triple negative breast cancer patient, which contains epithelial and mesenchymal subpopulations that we identified by flow cytometry following staining for the epithelial antigens EpCAM and CD49f (Figure 5F). We isolated these two subpopulations by FACS, then treated the isolated subpopulations with $30 \mathrm{nM}$ trametinib for 24 hours. These two subpopulations both dynamically respond to trametinib treatment, with a total transcriptional response of $15 \%$ of the transcriptome in the EpCAM+ subpopulation, and $9.1 \%$ of the transcriptome in the EpCAM- subpopulation (Figure 5G). We identified 280 genes that were commonly upregulated in both subpopulations in response to acute trametinib treatment, as well as 594 genes that were uniquely upregulated in the EpCAM+ subpopulation, and 369 genes that were uniquely upregulated in the EpCAM- subpopulation. 
The kinome rapidly adapts to trametinib treatment in both the EpCAM+ and EpCAM- subpopulation, with the upregulation of several kinases in both the EpCAM+ and EpCAM- subpopulation (Figure $5 \mathrm{H}$ ) highlighted in grey, as well as several kinases that are uniquely upregulated in the EpCAM+ subpopulation (blue) or the EpCAM- subpopulation (red). Immunoblots of both the EpCAM+ and EpCAMsubpopulations following trametinib demonstrate that these subpopulations both upregulate the collagenactivated receptor tyrosine kinase DDR1 (Figure 5I). However, the EpCAM+ subpopulation uniquely upregulates FGFR2, an RTK upregulated in several basal-like cell lines, whereas the EpCAMsubpopulation uniquely upregulated PDGFR $\beta$, an RTK upregulated in several claudin-low cell lines.

\section{Transcriptional Response to Trametinib is Associated with Epigenetic Remodeling}

Even though they were isolated from the same patient, the unique responses in the EpCAM+ and EpCAM- subpopulation suggest that differences in epigenetic imprinting may determine response to trametinib. Therefore, we performed a survey of epigenetic marks of transcriptional activation in SUM-159 cells to determine if epigenetic remodeling in response to trametinib regulates the transcriptional response to drug. DDR1 expression has been shown to be transcriptionally responsive in both basal-like and claudin-low breast cancer cell lines, so we examined the DDR1 locus in SUM-159PT cells to define the role of epigenetic remodeling on target gene transcription at this site. We observed the enrichment of $\mathrm{H} 3 \mathrm{~K} 4 \mathrm{me} 3$ at the transcription start site of DDR1, which has been shown in previous studies to delineate the promoter region of actively transcribed genes (Figure 6A, navy) (Guenther et al., 2007). Furthermore, we identified an enhancer region enriched in $\mathrm{H} 3 \mathrm{~K} 4 \mathrm{me} 1$ approximately $20 \mathrm{~kb}$ from the promoter region (Figure 6A, yellow). Following $24 \mathrm{hr}$ treatment of SUM-159PT cells with 100 trametinib, histone acetyltransferase p300 binding is enriched at promoter and enhancer regions in the trametinib treated sample relative to the DMSO control (Figure 6, cyan). Furthermore, we observed increased H3K27ac (Figure 6A, green) in trametinib treated samples at p300 binding sites, likely the result of increased binding and histone acetylation by p300. Furthermore, we identified increased binding of the transcriptional co-activator BRD4 at sites of increased H3K27ac (A. Dey et al., 2003; Jang et al., 2005). In response to trametinib treatment, we observed the enrichment of p300 binding at the top fifty 

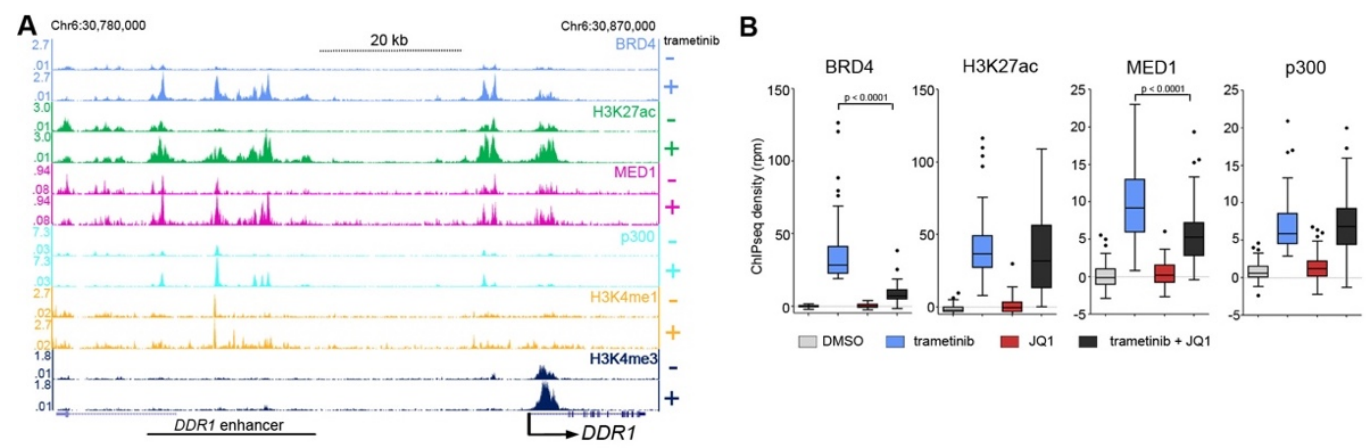

C
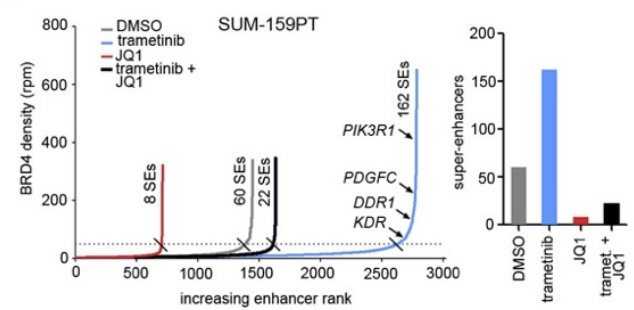

D

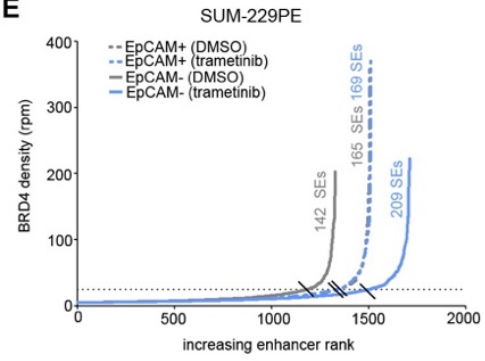

$\mathbf{F}$
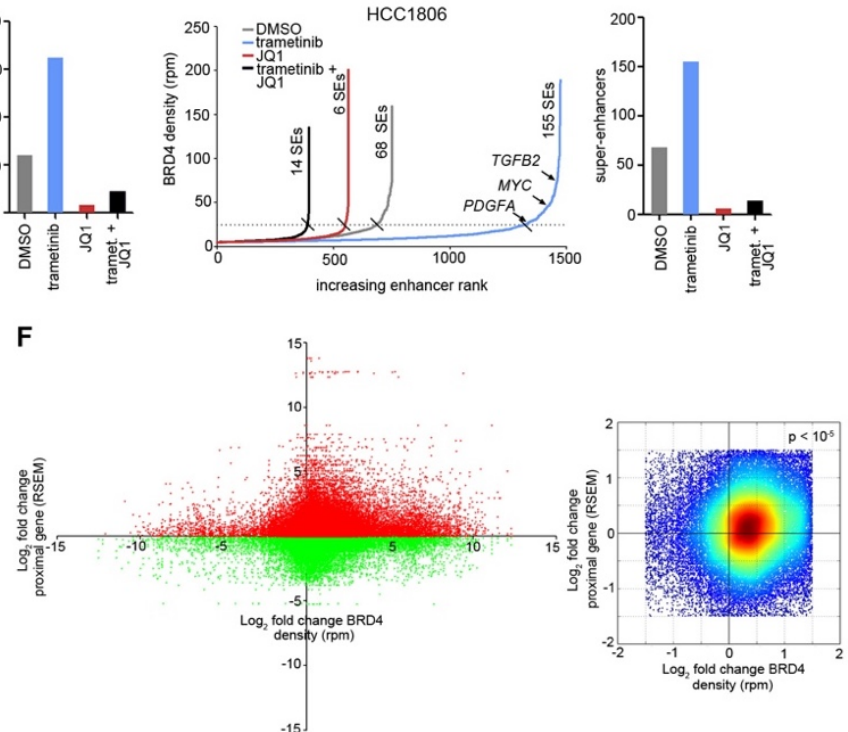

$\mathbf{G}$

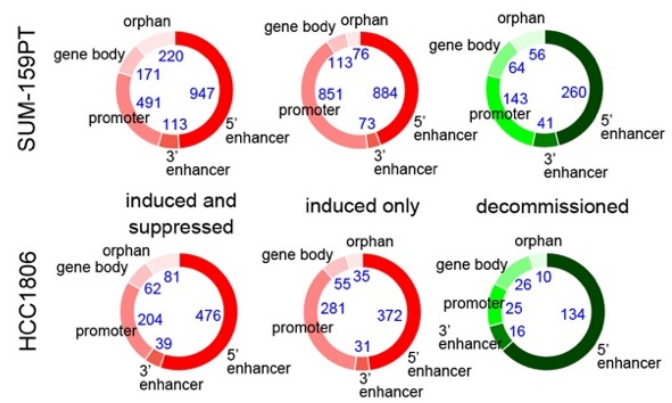

H

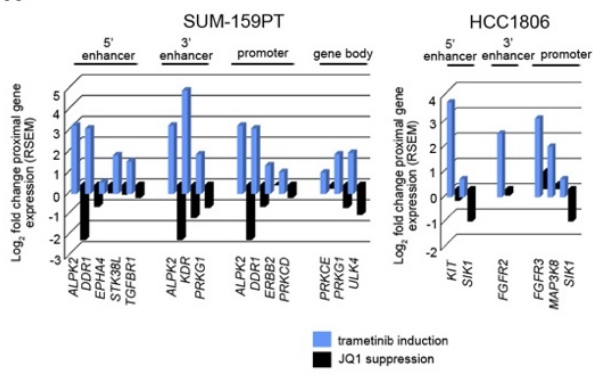

Figure 6. Transcriptional Response to Trametinib is Associated with Chromatin Remodeling in TNBC ${ }^{3}$. (A) ChIP-Seq at DDR1 locus for BRD4 (light blue), H3K27ac (green), Mediator (pink), p300 (cyan), H3K4me1 (yellow), H3K4me3 (navy) in SUM-159 cells following treatment with DMSO (-) or $100 \mathrm{nM}$ trametinib for 24 hours (+). (B) Density of BRD4, H3K27ac, MED1, and p300 peaks at 50 highest ranking BRD4 peaks following $24 \mathrm{hr}$ treatment with DMSO, $100 \mathrm{nM}$ trametinib, $300 \mathrm{nM}$ JQ1, or combination of JQ1 and trametinib. Quantification of BRD4 density at enhancer peaks following $24 \mathrm{hr}$ treatment with DMSO, trametinib, JQ1 or the combination in SUM-159PT (C) or HCC1806 (D). (E) Quantification of enhancers in EpCAM+ (blue) and EpCAM- (grey) subpopulations by BRD4 density following $24 \mathrm{hr}$ treatment with DMSO (dotted line) or $30 \mathrm{nM}$ trametinib (solid line). (F) Fold-change of BRD4 peak density relative to the transcription of target gene $+/-200 \mathrm{~kb}$ from peak following $24 \mathrm{hr}$ treatment with $100 \mathrm{nM}$ trametinib compared to DMSO control. (G) Classification of BRD4 peak location in SUM-159PT and HCC1806 for peaks that are induced greater than two-fold with trametinib treatment and suppressed greater than two-fold with JQ1 in combination, induced greater than two-fold with trametinib treatment and suppressed greater than two-fold with JQ1 in combination, or lost with trametinib treatment. $(\mathrm{H})$ Transcriptional response of indicated kinases associated with BRD4 peaks greater than two-fold with trametinib treatment and suppressed greater than two-fold with JQ1 in combination.

${ }^{3}$ Figure adapted from (Zawistowski et al., 2017) 
largest enhancer peaks (Figure 6B, blue), relative to the DMSO control (Figure 6B, grey). Accordingly, we identified increased H3K27ac and BRD4 binding concomitant with increased p300 binding at these enhancer peaks following trametinib treatment (Figure 6B, blue) relative to control (Figure 6B, grey). However, increased BRD4 binding in response to trametinib is abrogated when the BRD4 inhibitor JQ1 is added in combination with trametinib (Figure 6B, black). We defined enhancer peaks genome wide by BRD4 peaks within $200 \mathrm{~kb} 5^{\prime}$ of the TSS or $200 \mathrm{~kb} 3^{\prime}$ of the TES, but outside the promoter region $+/-5 \mathrm{~kb}$ from the TSS. We observed robust remodeling of the enhancer landscape in response to trametinib treatment, which induced the formation of de novo enhancer regions enriched in BRD4 binding. We identified 1445 BRD4-enriched enhancers in SUM-159 cells following treatment with the DMSO control, which increases to 2782 enhancers following treatment with trametinib (Figure 6C). Furthermore, ranking enhancers by BRD4 density allowed us to identify super enhancers (Lovén et al., 2013; Parker et al., 2013; Whyte et al., 2013), which increased from 60 super enhancers in the DMSO control to 162 super enhancers in trametinib treated sample. Similar to the effect we observed at the largest BRD4 peaks in Figure 6B, we observed decommissioning of both enhancers and super enhancers when JQ1 was combined with trametinib treatment. We identified 22 super enhancers in samples treated with both JQ1 and trametinib, compared to the 60 super enhancers we identified in the DMSO control and the 162 super enhancers we identified following treatment with trametinib alone. Epigenetic remodeling in response to trametinib treatment was also observed in the basal-like breast cancer cell line HCC1806, which created de novo enhancers following treatment, and were abrogated in combination with JQ1. The formation of de novo enhancers was identified in the EpCAM+ and EpCAM- subpopulations as well, which suggests that the recruitment of BRD4 to enhancer regions in response to trametinib treatment may be a general mechanism to stimulate growth in TNBC in response to the loss of MAPK pathway activation. Therefore, we examined the correlation between the recruitment of BRD4 to enhancer regions, and the transcription of the gene most proximal to the peak. Increased gene expression was positively correlated with increased BRD4 recruitment, suggesting that BRD4 binding may increase transcription activation of target genes (Figure 6F). However, we also observed increased binding of BRD4 at genes that were downregulated in response to trametinib treatment, which indicates that these BRD4 enriched enhancers may not be regulating the expression of the most proximal gene, or may play a role in the repression of 
transcription. We hypothesized that epigenetic remodeling of BRD4 peaks in promoter or enhancer regions may have differential effects on transcriptional response, so we classified BRD4 peaks by distance relative to the TSS. BRD4 peaks were classified as a 5' enhancer peak if it was located between $-200 \mathrm{~kb}$ to $-5 \mathrm{~kb}$ relative to the TSS, a 3' enhancer peak if it was located 0 to $200 \mathrm{~kb}$ from the TES, a promoter peak if it was located $+/-5 \mathrm{~kb}$ from the TSS, a gene body peak if it was located in gene exons or introns, or an orphan peak if no classification applied. This scheme was used to classify peaks with the following response patterns: induced by trametinib and suppressed by the addition of JQ1, induced by trametinib but not suppressed by the addition of JQ1, or suppressed by trametinib alone (Figure 6G). In each of the three classes, BRD4 peaks were most commonly found in the 5' enhancer region, and this class accounts for approximately half of all BRD4 peaks induced in response to trametinib treatment in SUM-159 and HCC1806 cells. Interestingly, BRD4 peaks located in the promoter region of genes were strongly induced in response to trametinib treatment, but these peaks seemed to be more resistant to inhibition by JQ1, which suggests that bromodomain activity is required for binding at enhancers but not promoter regions. BRD4 peaks suppressed in response to trametinib represents approximately $25 \%$ of the total number of gained peaks, indicating that loss of BRD4 binding at these sites may facilitate BRD4 recruitment at enriched peaks. Previous studies demonstrate that decommissioning of BRD4 enhancer peaks allows rapid chromatin remodeling to finely tune the activation of target genes (J. D. Brown et al., 2014). Examination of BRD4 peaks associated with kinases activated in response to trametinib treatment in the claudin-low SUM-159 cell line and the basal-like HCC1806 cell line revealed that BRD4 binding increases in all peak classes, further suggesting that BRD4 contributes to the upregulation of target kinases (Figure 6H). These results indicate that genome-wide epigenetic remodeling of BRD4 density may regulate transcription of target kinases to reactivate growth signaling in the presence of trametinib.

\section{Transcriptional Activation in Response to Trametinib by Distal Enhancers}

We hypothesized that BRD4 recruitment to enhancer regions associated with kinases increased target kinase expression in response to trametinib treatment. Therefore, we assessed BRD4 recruitment to the DDR1 enhancer region by BRD4 ChIP-Seq following treatment of SUM-159PT cells 
A

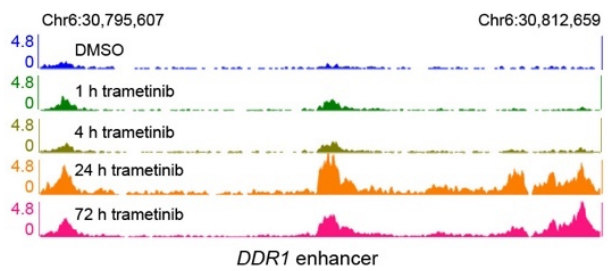

B

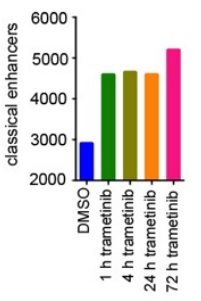

C

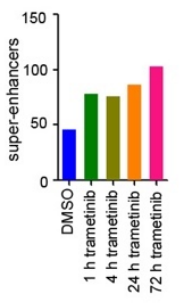

$100 \mathrm{nM}$ trametinib

Oh 1 h 4 h 8 h 24 h

$----\infty$ DDR1

$---\ldots$ MYC

--- ERK2

D
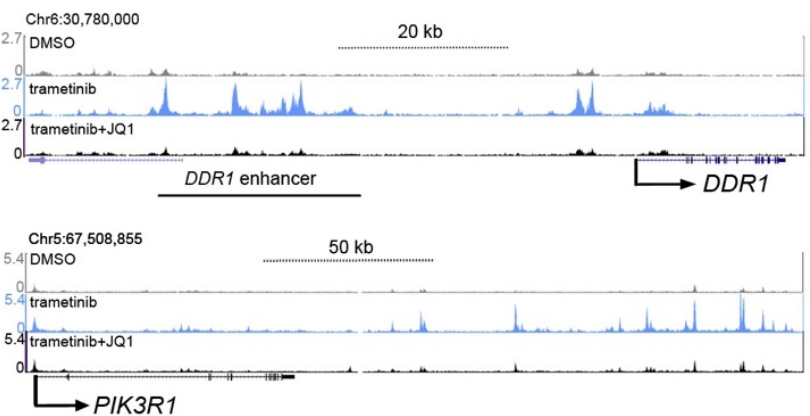

E

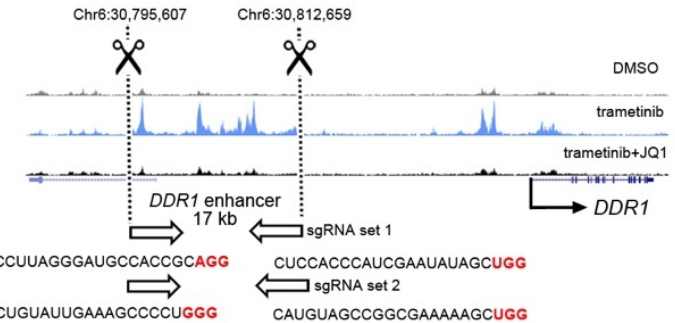

GCUCUGUAUUGAAAGCCCCUGGG CAUGUAGCCGGCGAAAAAGCUGG

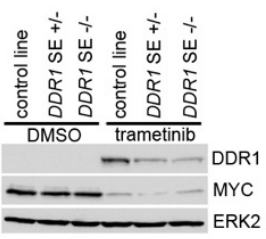

Figure 7. Regulation of Target Gene Expression by Distal Enhancers ${ }^{4}$. (A) ChIP-Seq for BRD4 binding at DDR1 enhancer region during treatment with $100 \mathrm{nM}$ trametinib. Enrichment can be observed by 1 hour of treatment (green), and reaches maximal levels at 24 hours (orange). (B) Analysis of genome-wide BRD4 ChIP-Seq demonstrates that recruitment to enhancers and super enhancers genome wide observed by 1 hour of treatment (green), but reaches maximal levels by 72 hours (pink). (C) Immunoblot for DDR1 and MYC during trametinib treatment demonstrates DDR1 increases concomitant with MYC loss. (D) ChIP-Seq for BRD4 reveals BRD4 recruitment at enhancer regions associated with the regulatory subunit of PI3K, PIK3R1, as well as the receptor tyrosine kinase KDR. (E) Two independent CRISPR sgRNAs were designed to knock out the enhancer associated DDR1. Immunoblot for DDR1 following treatment with trametinib demonstrates enhancer knock out abrogates DDR1 expression compared to sgRNA control.

${ }^{4}$ Figure adapted from (Zawistowski et al., 2017) 
with $100 \mathrm{nM}$ trametinib (Figure 7A) for $0 \mathrm{hr}$ (blue), $1 \mathrm{hr}$ (green), $4 \mathrm{hrs}$ (olive), 24 hrs (orange), and $72 \mathrm{hrs}$ (pink). BRD4 is recruited to the DDR1 enhancer region in as little as 1 hour of treatment with $100 \mathrm{nM}$ trametinib, and continues to increase to a maximal level at 24 hours. While BRD4 enrichment continues to persist at 72 hours of trametinib treatment, BRD4 recruitment is slightly diminished relative to the enrichment at 24 hours. Genome-wide recruitment of BRD4 to enhancers is observed after 1 hour of treatment with trametinib, but the total number of enhancer and superenhancer peaks continues to increase up to 72 hours of trametinib treatment. Furthermore, immunoblot analysis of DDR1 expression demonstrates that DDR1 levels increase concomitant with increased recruitment of BRD4 (Figure 7A). Increased expression of DDR1 was observable as early as 1 hour of trametinib treatment and maximal expression was reached by 24 hours (Figure 7C). We examined other superenhancers associated with kinases that were upregulated in response to trametinib, and identified the enrichment of BRD4 at the enhancer region near PIK3R1, a regulatory subunit of $\mathrm{PI} 3 \mathrm{~K}$, as well at the enhancer region near KDR (Figure 7D, blue). Furthermore, addition of the BRD4 inhibitor JQ1 in combination with trametinib abolished the recruitment of BRD4 at these enhancer regions (Figure 7D, black), suggesting that the addition of JQ1 is sufficient to block epigenetic remodeling and kinome reactivation in response to treatment. We hypothesized that the recruitment of BRD4 at the enhancer region of DDR1 activates expression of DDR, so we designed CRISPR sgRNA guides to knock out the DDR1 enhancer region and quantify DDR1 expression in the presence of trametinib without the enhancer. Following treatment with $100 \mathrm{nM}$ trametinib, homozygous knockout of the DDR1 enhancer significantly decreases expression of DDR1 relative to the non-targeting sgRNA control. These data indicate that the enhancer region of DDR1 activates transcription of DDR1, suggesting that epigenetic remodeling of this enhancer in response to trametinib is sufficient to activate transcription of receptor tyrosine kinases to reactivate MAPK.

Gene expression analysis of triple negative breast cancer cell lines demonstrates that the basallike molecular subtype is characterized by the baseline expression of epithelial genes, whereas the claudin-low molecular subtype is characterized by the baseline expression of mesenchymal genes. The phenotypic differences between these two molecular subtypes is further evident in the differential response to treatment with trametinib, which induces a transcriptional response that is unique to each molecular subtype. DESeq analysis of kinase transcription demonstrates that MAP2K6, FRK, and MYLK 
are significantly transcriptionally activated in basal-like breast cancer cell lines in response to trametinib relative to claudin-low cell lines. Furthermore, this analysis identified DCLK1, IP6K3 and NPR1 are significantly transcriptionally activated in claudin-low cell lines in response to trametinib relative to basallike cell lines. However, many kinases are commonly upregulated in both molecular subtypes, including $D D R 1$ and JAK1, which suggests that there is a global epigenetic response to MEK inhibition to stimulate pathway reactivation. Zawistowski and Bevill et al. demonstrate that epigenetic remodeling occurs in as little as 1 hour in response to trametinib treatment, and BRD4 is recruited to the 5' enhancer regions associated with transcriptionally activated kinases. Pathway reactivation by transcriptional upregulation has been observed in several other preclinical models in response to targeted therapy. Stratikopoulous and colleagues demonstrated that treatment of metastatic breast cancer with the PI3K inhibitor GDC0941 induces the expression of INSR, IGF1R, HER2 and HER3, which can be blocked by combining JQ1 (Stratikopoulos et al., 2015). A similar response was observed in HER2-positive breast cancer cell lines following treatment with the ERBB family kinase inhibitor, lapatinib. Treatment of HER2-positive breast cancer cell lines induced epigenetic remodeling and transcriptional activation of HER3, IGF1R, DDR1, MET and FGF family RTKs, which was blocked with JQ1 in combination with lapatinib (Stuhlmiller et al., 2015). These results indicate that epigenetic remodeling in response to targeted therapy is sufficient to upregulate expression of RTK and increase pathway activation in the presence of drug. However, the regulatory elements that control epigenetic remodeling remain unknown. 


\section{CHAPTER 4: DETERMINANTS OF TRAMETINIB RESPONSE IN TNBC}

Intratumor heterogeneity arising from genetic mutation and epigenetic remodeling represents a significant challenge to therapeutic durability and prevention of resistance. Deep sequencing of tumors has revealed that most mutations identified in the tumor mass could not be detected in all tumor regions (Gerlinger et al., 2012). This heterogeneity increases the likelihood that drug tolerant clones will persist throughout treatment and lead to relapse. Indeed, whole exome sequencing of circulating tumor DNA isolated from breast cancer patients during treatment revealed the enrichment of low frequency mutations that mediate therapeutic resistance (Murtaza et al., 2013). However, several studies have also observed a spectrum of phenotypes can emerge from tumor cells with similar genotypes (Chaidos et al., 2013; Wang et al., 2014). Therefore, many hypothesize that the epigenetic plasticity of cancer also contributes to the persistence of drug resistant cells (R. Brown et al., 2014; Dean et al., 2005; Easwaran et al., 2014). A recent study of circulating tumor cells revealed that these cells are not always committed to a single cell state, but can display a mixture of phenotypes (Yu et al., 2013). This study also showed that epithelial and mesenchymal subpopulations of circulating tumor cells were differentially sensitive to treatment with PI3K and MEK inhibitor. Recent work interrogating resistance to targeted therapy demonstrated that breast cancer cells can become drug tolerant by switching cell state (Risom et al., 2018). While tolerance can emerge from global remodeling that induces cell state switching, it can also be caused by the epigenetic activation of specific genes to stimulate resistance (Sharma et al., 2010). These results suggest that genetic heterogeneity and epigenetic plasticity play essential roles in mediating drug resistance and selection of drug tolerant phenotypes.

Triple negative breast cancer (TNBC) is a heterogenous disease clinically characterized by the absence of the estrogen, progesterone and HER2 receptors. Unlike other breast cancer subtypes, there are no FDA-approved targeted therapies currently available for triple negative breast cancer patients. These patients are treated with a combination of surgery, radiation therapy, and chemotherapy (Wahba and El-Hadaad, 2015). Clinical studies demonstrate that while triple-negative breast cancer patients have 
the best pathological complete response rates of any breast cancer subtype, TNBC patients with residual disease have the worst survival (Liedtke et al., 2008). Deep sequencing of TNBC patients revealed a broad distribution in the number of clonal subpopulations within individual tumors, suggesting that the genetic heterogeneity contributes to outcome (Shah et al., 2012). These results indicate that heterogeneity in TNBC breast cancer represents a significant obstacle to improvement of patient outcomes with current therapies. Therefore, identifying durable targeted therapies for triple-negative patients is essential to improving outcomes for patients with heterogeneous tumors.

Despite their initial promise, kinase inhibitors have often been ineffective as a monotherapy due to epigenetic remodeling and transcriptional activation that occurs in response to treatment. The Cancer Genome Atlas Project sequenced 510 breast cancer tumors, which identified the overexpression of EGFR, KRAS, and BRAF (Cancer Genome Atlas Network, 2012). As a result, there are ongoing clinical trials examining the safety and efficacy of the inhibitors of the MAPK signaling pathway in breast cancer patients, including MEK inhibitors selumetinib and trametinib. A window-of-opportunity trial enrolling triplenegative breast cancer patients treated with trametinib demonstrated that patients treated with trametinib for seven days rapidly respond to treatment, increasing expression of receptor tyrosine kinases (RTKs) to reactivate MAP kinase signaling (Zawistowski et al., 2017). Similar work examined the adaptive response to AKT inhibition, which revealed the upregulation of receptor tyrosine kinases in several preclinical models (Chandarlapaty et al., 2011). An analogous observation was made following treatment of HER2positive breast cancer with the ERBB-family kinase inhibitor lapatinib, but combining a BET bromodomain inhibitor with lapatinib abrogated transcriptional activation and produced a synergistic effect (Stuhlmiller et al., 2015). ChIP-Seq experiments in triple negative breast cancer demonstrated that treatment with targeted therapy increases acetylation histone $\mathrm{H} 3$ at lysine 27 (H3K27ac) concomitant with the recruitment of the histone acetyltransferase p300 and increases binding of the BET bromodomain protein BRD4 (Zawistowski et al., 2017). These results suggest that epigenetic remodeling by increased $\mathrm{H} 3 \mathrm{~K} 27 \mathrm{ac}$ and BRD4 recruitment play an essential role in transcriptional activation in response to targeted therapy.

We aim to understand the regulatory elements that control epigenetic remodeling in response to targeted therapy in heterogenous breast cancers by studying the response in epithelial and mesenchymal 
subpopulations of the heterogeneous TNBC cell line SUM-229PE. These cells were isolated from the pleural effusion of a breast cancer patient following treatment with chemotherapy, and express cytokeratins consistent with their origin from luminal breast epithelial cells (Forozan et al., 1999). A comprehensive analysis of heterogeneity in breast cancer cell lines identified two distinct subpopulations with unique morphologies that differentially express epithelial markers (Keller et al., 2010). Furthermore, an siRNA screen in the epithelial and mesenchymal subpopulations of SUM-229 cells discovered that SMARCD3, a regulatory subunit of the SWI/SNF chromatin remodeling complex, is necessary for the maintenance of phenotype, suggesting the phenotype is epigenetically controlled (Jordan et al., 2013). This work aims to understand how these distinct epithelial and mesenchymal subpopulations epigenetically control normal gene expression and dictate the response to therapy, to provide insight into how resistance may emerge during treatment with targeted therapy.

\section{Subpopulations in TNBC Have Similar Genotypes with Distinct Phenotypes}

The cell line SUM-229PE was isolated from the pleural effusion of a triple negative breast cancer patient, and contains cells with epithelial and mesenchymal morphologies. Immunostaining of SUM229PE cells (Figure 8A) reveals a subpopulation of cells with an elongated phenotype, prominent filamentous actin fibers (green) and perinuclear vimentin (red), as well as a subpopulation of cells with a rounded phenotype that stain for elevated levels of the epithelial marker EpCAM (blue). The SUM-229PE cell line was analyzed by flow cytometry (Figure 8B) following staining for epithelial cell surface markers EpCAM and CD49f (integrin $\alpha 6$ ). We observed a subpopulation of cells that stained positive for EpCAM and CD49f (POS) and a subpopulation of cells that stained negative for EpCAM and CD49f (NEG). The POS and NEG subpopulations were isolated by FACS to greater than $99.9 \%$ purity, then cultured for up to six weeks after isolation. Following six weeks of culture after isolation, less than $1 \%$ of the NEG subpopulation staining positive for EpCAM and CD49f. We previously reported that whole exome sequencing of POS and NEG subpopulations identified 8,373 single nucleotide variants in each 

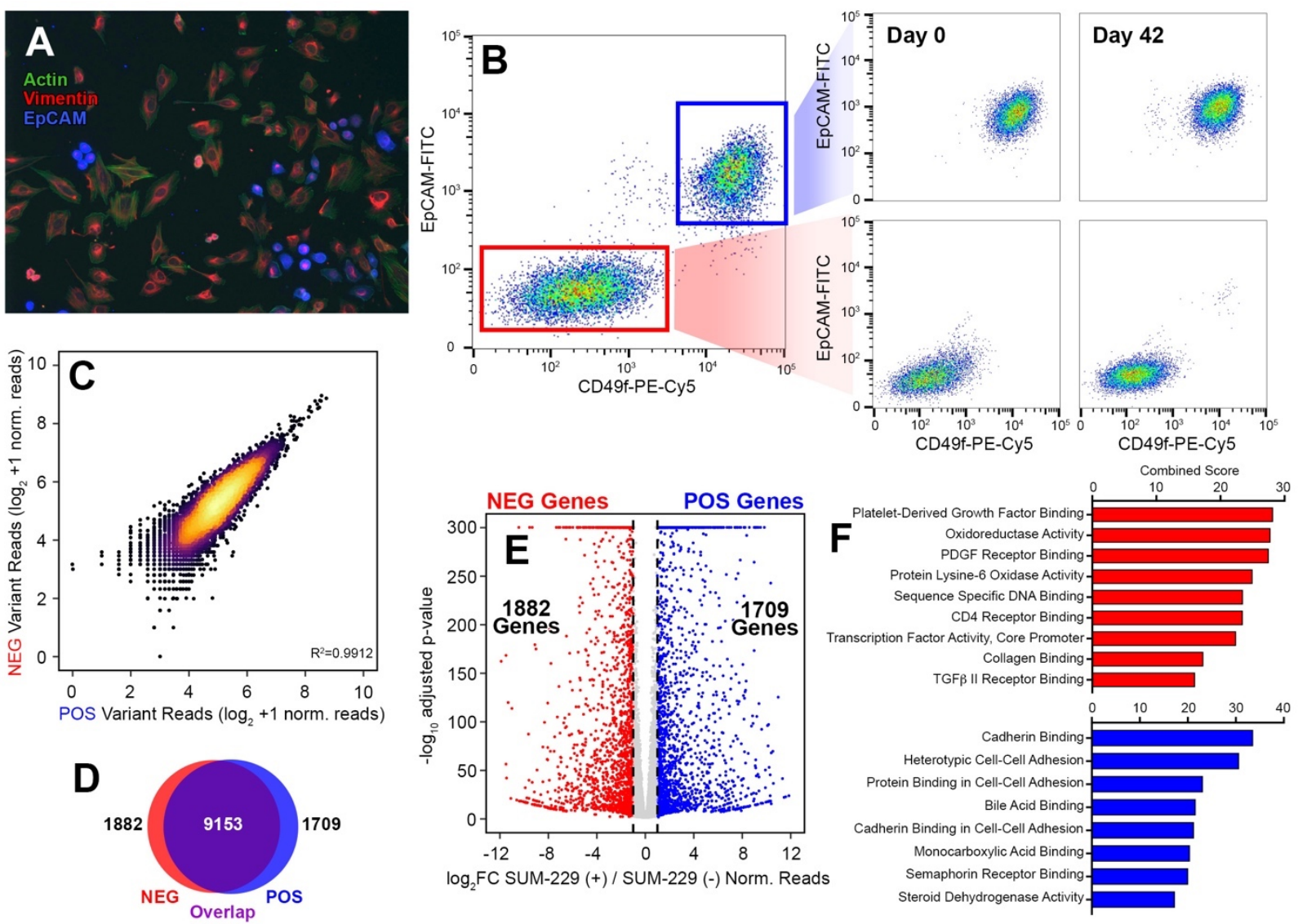

Figure 8. Genetically Similar Subpopulations in TNBC Express Distinct Phenotypes. (A) Immunofluorescence staining of SUM-229 parental culture for EpCAM (blue), Actin (green), and Vimentin (red). (B) Flow cytometry of SUM-229 parental culture containing an epithelial subpopulation (POS) that stains positive for epithelial antigens EpCAM and CD49f, and a mesenchymal subpopulation (NEG) the stains negative for EpCAM and CD49f. POS and NEG subpopulations were isolated by FACS, and analyzed after 42 days of continuous culture. (C) Comparison of total reads containing non-synonymous mutations that were identified by whole exome sequencing of POS (x) and NEG (y) subpopulations. (D) Comparative analysis of gene expression in the isolated subpopulations identified 1865 genes that have greater than two-fold expression in the NEG subpopulation compared to the POS subpopulation, with a p-value less than 0.05 . Similarly, 1666 genes have greater than two-fold expression in the POS compared to the NEG, with a p-value less than 0.05. (E) DESeq analysis of gene expression in POS and NEG calculates significance of differential expression. Dashed lines indicate log2 fold change greater than 1, which is highlighted in POS (blue) and NEG (red). (F) Gene ontology analysis of the molecular function of differentially expressed genes identified by DESeq in NEG (red) and POS (blue) using the Molecular Function GO terms in the Enrichr database. Combined score calculated using Enrichr database; log of $p$-value multiplied by z-score deviation from expected rank. 
subpopulation compared to the reference genome, with no SNVs unique to either subpopulation (Zawistowski et al., 2017). We reanalyzed the whole-exome sequencing data using a germline rather than somatic mutation caller, and compared the total reads of all variants identified in POS and NEG cells (Figure 8C). The germline variant caller identified 133 mutated genes that were unique to either POS or NEG subpopulations (Table 2). Furthermore, we performed karyotyping of the POS and NEG subpopulations, which revealed that the two subpopulations share similar ploidy and many of the same chromosomal aberrations, but the NEG subpopulation contains a higher frequency of dicentric chromosomes (Figure 9, Table 3). Despite the genomic similarity of the two subpopulations, we hypothesized that the distinct phenotypes we observed in immunofluorescence and flow cytometry assays were the result of distinct transcriptional programs in POS and NEG cells. Therefore, we performed RNA-Seq on POS and NEG cells to identify the determinants of these differential phenotypes. We captured 12744 genes with a minimum of 25 reads in either subpopulation, and classified $29 \%$ of the transcriptome as differentially expressed between subpopulations, with a minimum two-fold difference in expression and a p-value of less than 0.05 (Figure 8D and 8E). To further study these genes, we interrogated the molecular function of the 1882 genes differentially expressed in the NEG subpopulation, and the 1709 POS differentially expressed in the POS subpopulation using the Enrichr gene ontology database (Chen et al., 2013; Kuleshov et al., 2016). Genes specifically expressed in the NEG subpopulation are significantly enriched for GO terms associated with a mesenchymal phenotype, including PDGF receptor binding, collagen binding, and TGF $\beta$ receptor binding (Figure 8F). Examination of the genes underlying the enrichment of $\mathrm{GO}$ terms revealed increased expression of PDGFRA, PDGFRB, TGFBR3, TGFB1, FN1, and ITGA11 (Figure 9C-F). Genes specifically expressed in the POS subpopulation are significantly enriched for GO terms associated with an epithelial phenotype, including cadherin binding and cell-cell adhesion. The genes identified in the POS enriched GO terms showed increased expression of $C D H 1, E P C A M, D S G 2$, and KRT18 in the POS subpopulation (Figure 9G-I). To further define the phenotypes of the POS and NEG subpopulations, we used two genesets from the MSigDB developed by Charafe-Jauffret et al., which distinguishes mesenchymal and basal-epithelial subtypes using RNA-Seq data collected from 31 different breast cancer cell lines (Charafe-Jauffret et al., 2006). A heatmap (Figure 9J) of the log2- transformed, row-mean centered values from biological 


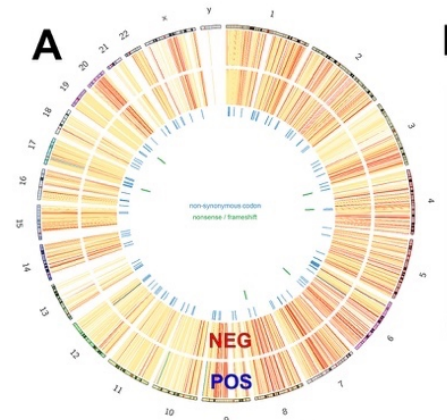

B
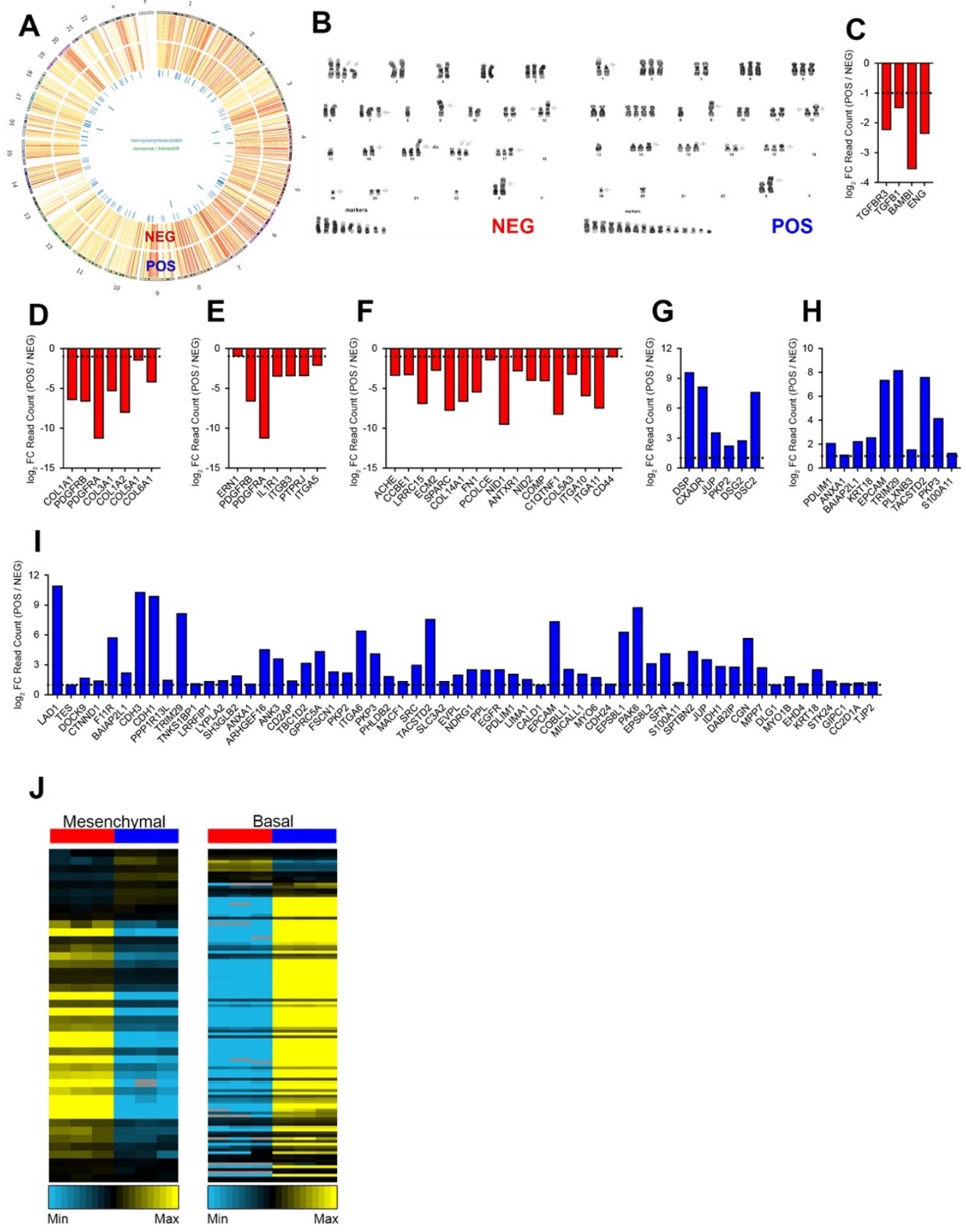

Figure 9: Supplemental Figure: Genetically Similar Subpopulations in TNBC Express Distinct Phenotypes. (A) Circos plot of single nucleotide variants identified in POS and NEG subpopulations relative to reference genome hg19. Heatmap displays allelic frequency of variant calls (outer band). Variant mismatch identified with blue mark (inner ring). (B) Cytogenic analysis of POS and NEG subpopulations, high frequency of dicentric chromosomes observed in NEG chromosomes (arrows). Cytogenetic abnormalities listed in Table 3. (C) Differential gene expression of genes identified in NEG subpopulation by TGF $\beta$ II Receptor GO term. Values represent the $\log _{2}$ transformation of ( POS / NEG ) average gene expression for three replicate RNA-Seq experiments. (D)

Differential gene expression of genes identified in NEG subpopulation by PDGF Binding GO term. (E) Differential gene expression of genes identified in NEG subpopulation by PDGFR Binding GO term. (F) Differential gene expression of genes identified in NEG subpopulation by Collagen Binding GO term. (G) Differential gene expression of genes identified in POS subpopulation by Heterotypic Cell-Cell Adhesion GO term. (H) Differential gene expression of genes identified in POS subpopulation by Cell-Cell Adhesion GO term. (I) Differential gene expression of genes identified in POS subpopulation by Cadherin Binding GO term. (J) Heatmap of gene expression of genes in Mesenchymal and Basal genesets developed by Charafe-Jauffret et al. and submitted to MSigDB. Heatmap displays $\log _{2}$ transformed, mean-centered, normalized gene expression of triplicate RNA-Seq values in NEG (red) and POS (blue). Max expression shown in yellow, minimum in cyan. 


\begin{tabular}{|c|c|c|c|c|c|c|c|c|}
\hline Gene & NEG & POS & Gene & NEG & POS & Gene & NEG & POS \\
\hline ACRV1 & 2.6 & 1.9 & HS6ST1 & 300.0 & 2672.1 & PEAR1 & 11.9 & 71.4 \\
\hline AHNAK & 15110.8 & 17793.5 & IGSF5 & 0.4 & 0.0 & PIK3C2G & 0.0 & 0.9 \\
\hline AKAP10 & 495.1 & 623.3 & IL17RD & 10.8 & 18.7 & PLIN4 & 41.2 & 168.8 \\
\hline AKAP11 & 763.6 & 933.6 & IL27 & 0.0 & 0.0 & POLR2J3 & 350.6 & 591.7 \\
\hline AKAP3 & 1.5 & 0.5 & IL4R & 776.0 & 1157.0 & POTED & 0.0 & 0.0 \\
\hline ANKRD30B & 5.0 & 0.2 & ITGAL & 0.2 & 1.4 & PRAMEF11 & 0.0 & 0.0 \\
\hline ANKRD36C & $\# \mathrm{~N} / \mathrm{A}$ & \#N/A & KCNJ12 & 3.3 & 0.5 & PRB2 & 0.8 & 0.0 \\
\hline ARSD & 114.0 & 324.3 & KDM1B & 359.0 & 571.7 & PRIM2 & 485.8 & 491.6 \\
\hline C12orf63 & 3.1 & 0.9 & KREMEN1 & 20.8 & 1035.5 & PRPF4B & 1551.9 & 1719.1 \\
\hline $\mathrm{C7}$ & 3.5 & 0.0 & KRTAP4-7 & 0.0 & 0.0 & PSORS1C2 & 1.1 & 5.0 \\
\hline C8orf89 & \#N/A & \#N/A & KRTAP9-2 & 0.0 & 0.0 & PTPRH & 164.4 & 159.2 \\
\hline $\mathrm{CDH}_{20}$ & 0.6 & 0.0 & LEFTY1 & 1.5 & 5.7 & REC8 & 31.8 & 19.2 \\
\hline CSTL1 & 0.9 & 0.6 & LEPRE1 & 2538.9 & 776.4 & RGPD3 & 14.4 & 14.3 \\
\hline CTAGE6 & 4.0 & 27.5 & LILRB3 & 18.2 & 2.4 & RGPD8 & 6.3 & 14.5 \\
\hline CTBP2 & 1752.7 & 1654.0 & LOC 10099663 & $\# N / A$ & $\# N / A$ & RPA1 & 2404.9 & 2709.9 \\
\hline СTBS & 268.0 & 279.9 & LRIT3 & 0.4 & 0.4 & RREB1 & 591.2 & 1093.1 \\
\hline $\mathrm{CTSH}$ & 159.7 & 3835.5 & LRRC37A2 & 253.6 & 359.6 & SENP3 & 1934.9 & 1773.6 \\
\hline DIAPH3 & 741.7 & 891.9 & LTBP4 & 1093.1 & 496.1 & SERPINB6 & 1467.2 & 1471.2 \\
\hline DNAl1 & 1.8 & 2.2 & MLL3 & 674.3 & 1557.8 & SETD1A & 1651.0 & 1076.1 \\
\hline DPYSL5 & 0.4 & 0.0 & MUC12 & 6.2 & 5.3 & SFRP4 & 0.4 & 0.4 \\
\hline DSC1 & 0.0 & 0.4 & MUC16 & 0.0 & 0.2 & $\mathrm{SH} 2 \mathrm{~B} 1$ & 935.5 & 758.7 \\
\hline DSP & 16.6 & 18555.2 & MUC3A & $\# N / A$ & $\# \mathrm{~N} / \mathrm{A}$ & SIRT5 & 186.7 & 354.3 \\
\hline DTX2 & 391.6 & 569.0 & MUC4 & 0.5 & 1.6 & SKA3 & 601.1 & 409.0 \\
\hline FAM 104B & 86.0 & 87.8 & MUC5AC & $\# N / A$ & $\# \mathrm{~N} / \mathrm{A}$ & SLC25A5 & 7465.2 & 7950.7 \\
\hline FAM157B & 2.6 & 13.5 & MUC5B & 1.3 & 0.4 & SLC37A1 & 1.1 & 192.0 \\
\hline FAM22G & 4.5 & 11.9 & MUC6 & 1.1 & 0.4 & SMG6 & 359.1 & 508.9 \\
\hline FMO3 & 2.8 & 0.0 & MYO1H & 1.3 & 0.8 & SULT1A2 & 9.0 & 14.0 \\
\hline GGN & 22.0 & 13.5 & MYO5B & 10.6 & 209.4 & TBC1D26 & 0.6 & 0.0 \\
\hline GGT1 & 24.8 & 16.2 & NBPF1 & 513.1 & 392.8 & TDG & 584.8 & 979.6 \\
\hline GMPR & 26.2 & 311.9 & NHEDC1 & 9.7 & 6.3 & TDRD15 & \#N/A & \#N/A \\
\hline GOLGA1 & 450.7 & 662.4 & NQO2 & 421.2 & 499.8 & TECPR2 & 317.5 & 331.1 \\
\hline GOLGA6L4 & $\# N / A$ & $\# \mathrm{~N} / \mathrm{A}$ & OBSCN & 63.7 & 426.0 & TEKT4 & 0.4 & 0.5 \\
\hline GRM1 & 1.5 & 1.6 & OR2T27 & 0.0 & 0.0 & THOP1 & 1171.2 & 837.4 \\
\hline GSTZ1 & 129.6 & 445.1 & OR2T33 & 0.0 & 0.0 & TMEM8A & 796.4 & 1018.4 \\
\hline GTF3C1 & 4106.4 & 3209.2 & OR2T35 & 0.0 & 0.0 & TNN & 0.0 & 0.0 \\
\hline GXYLT1 & 666.0 & 572.3 & OR2T8 & 0.0 & 0.0 & TPCN2 & 193.0 & 255.9 \\
\hline HADHB & 1821.8 & 2173.9 & OR4C12 & 0.0 & 0.0 & TSPAN17 & 786.9 & 1614.2 \\
\hline HLA-A.4 & $\# N / A$ & \#N/A & OR5H15 & 0.0 & 0.0 & TSPYL1 & 1007.5 & 1454.3 \\
\hline HLA-DQA1 & 0.0 & 0.0 & OR8B3 & 0.0 & 0.0 & TTN & 130.3 & 216.3 \\
\hline HLA-DRB1 & 47.2 & 0.0 & PABPC 1 & 66860.2 & 56232.5 & USP6 & 42.1 & 28.1 \\
\hline HLA-DRB5 & 6.4 & 0.2 & PABPC 3 & 5584.0 & 4990.9 & VCX & 0.0 & 0.0 \\
\hline HMCN2 & $\# \mathrm{~N} / \mathrm{A}$ & $\# \mathrm{~N} / \mathrm{A}$ & PAD 14 & 0.0 & 2.1 & WDR69 & 45.4 & 263.8 \\
\hline HNRNPCL4 & $\# N / A$ & $\# \mathrm{~N} / \mathrm{A}$ & PCDHA10 & 28.0 & 22.6 & ZNF141 & 83.8 & 9.3 \\
\hline \multirow[t]{2}{*}{ HOXD8 } & 1.1 & 7.5 & PDE4DIP & 1419.3 & 2302.4 & ZNF595 & 54.1 & 24.7 \\
\hline & & & & & & ZNF717 & 81.1 & 8.0 \\
\hline
\end{tabular}

Table 2. Gene Expression of Differentially Mutated Genes Identified by Whole Exome Sequencing. Analysis of whole exome sequencing of POS and NEG subpopulations using the FreeBayes variant caller identified 133 genes with differential genotypes and confidence score greater than Q30. Genes listed with the mean expression of biological triplicate RNA-Seq of NEG and POS subpopulations. Expression listed as \#N/A if the variant gene call matches no known aliases in RNA-Seq expression data. 


\begin{tabular}{|c|c|c|}
\hline Chr & NEG & POS \\
\hline 1 & \multirow{4}{*}{+} & del $p$ \\
\hline 2 & & + \\
\hline 3 & & \\
\hline 4 & & + \\
\hline 5 & \multirow[t]{2}{*}{+} & \multirow[t]{2}{*}{+} \\
\hline 6 & & \\
\hline 7 & \multirow[t]{2}{*}{ derivative } & \multirow[t]{2}{*}{++} \\
\hline 8 & & \\
\hline 9 & \multirow[t]{3}{*}{ add $p$} & \multirow[t]{3}{*}{ add $p$} \\
\hline 10 & & \\
\hline 11 & & \\
\hline 12 & \multirow[t]{2}{*}{ iso $\mathrm{q}$} & \multirow[t]{2}{*}{ iso $\mathrm{q}$} \\
\hline 13 & & \\
\hline 14 & derivative & derivative \\
\hline 15 & derivative & derivative \\
\hline 16 & \multirow{7}{*}{ derivative } & derivative \\
\hline 17 & & \multirow[t]{6}{*}{ derivative } \\
\hline 18 & & \\
\hline 19 & & \\
\hline 20 & & \\
\hline 21 & & \\
\hline 22 & & \\
\hline$x$ & \multirow[t]{2}{*}{ add $p$} & \multirow[t]{2}{*}{ add $p$} \\
\hline Y & & \\
\hline \multirow[t]{2}{*}{ Chr \# } & $75 \%(53-61)$ & $90 \%(58-66)$ \\
\hline & $25 \%$ tet $(>100)$ & $10 \%$ tet $(>100)$ \\
\hline Dicentric & $15 \%$ & \\
\hline
\end{tabular}

Table 3. Chromosomal Aberrations Identified by Karyotyping of NEG and POS Subpopulations. Cytogenic analysis was performed on twenty G-banded metaphase spreads. "+" indicates the detection of additional copy of the corresponding chromosome, "derivative" indicates a chromosomal fragment was identified. "add" or "del" indicates that a chromosomal arm of unusual length was detected. Chromosomal arm is indicated with " $p$ " or " $q$ " where applicable. The "iso" notation indicates that an isochromosome was identified. 
triplicate of POS (blue) and NEG (red) RNA-Seq shows distinct enrichment of the genes in the mesenchymal geneset in the NEG subpopulation (red), and enrichment of the basal geneset in the POS subpopulation (blue). These data demonstrate that despite their genomic similarities, the differentially expressed genes in the POS and NEG subpopulations determine the epithelial and mesenchymal phenotypes observed in the mixed population.

\section{Epithelial and Mesenchymal Phenotypes are Epigenetically Imprinted}

The genomic similarities in the POS and NEG subpopulations led us to hypothesize that the differentially expressed genes were epigenetically imprinted. Therefore, we performed ChIP-Seq for transcriptional activation marks histone $\mathrm{H} 3$ lysine 27 acetylation (H3K27ac) and bromodomain-containing protein 4 (BRD4) binding in POS and NEG subpopulations. We used MACS to identify 16608 H3K27ac peaks with a minimum of 5 reads in either the POS or NEG subpopulations, with 4388 peaks (26\%) greater than two-fold enriched in the POS subpopulation and 5959 peaks (36\%) greater than two-fold enriched in the NEG subpopulation. Using MACS, these peaks were classified by the type of regulatory element and annotated to nearby genes. This comparison revealed enrichment of H3K27ac density at peaks near the TWIST1 and ZEB1 loci (red) in the NEG subpopulation relative to the POS subpopulation (Figure 10A). TWIST1 and ZEB1 are transcription factors specifically expressed in mesenchymal cells that have been shown to be essential regulators of the mesenchymal phenotype (Polyak and Weinberg, 2009). Furthermore, we identified enrichment of H3K27ac density at peaks near the epithelial-specific transcription factors TP63 and ELF3 loci (blue) (Blick et al., 2008; Eckel et al., 2003). Examination of the TWIST1 locus (Figure 10C) demonstrates specific enrichment of H3K27ac density in the NEG subpopulation (red) surrounding the TWIST1 transcription start site (TSS), compared to the POS subpopulation (blue). When comparing the POS and NEG subpopulations at the ELF3 locus (Figure 10C), however, the opposite is true: we observe specific enrichment of H3K27ac density in the POS subpopulation (blue) at the TSS of ELF3 relative to the NEG subpopulation (red). These results suggest that epigenetic regulators of transcriptional activation are specifically localized at the TSS of transcription factors that help determine phenotype. We further investigated the epigenetic regulation of transcriptional activity by comparing the localization of the histone-acetylation reader BRD4 in POS and NEG 

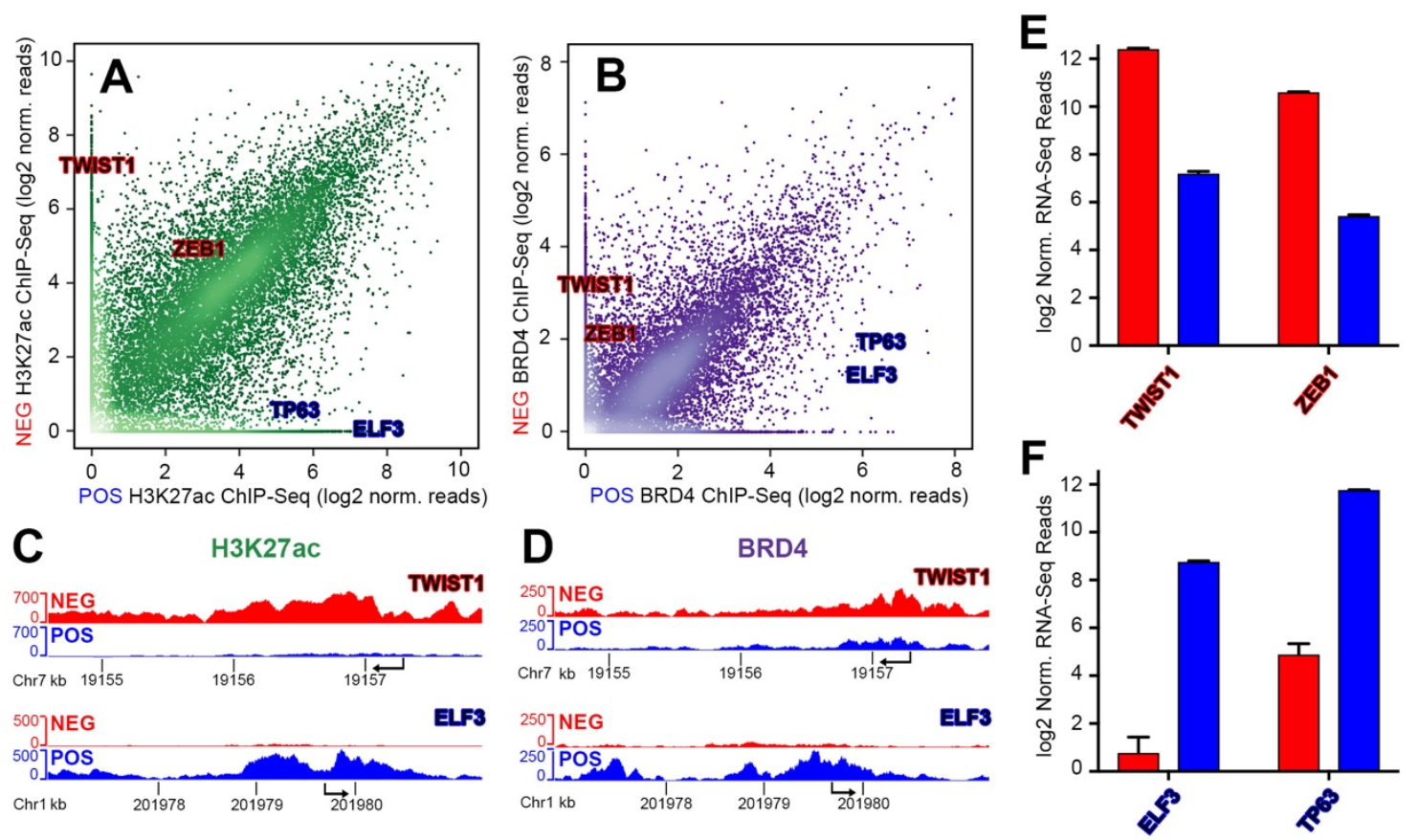

Figure 10. Epigenetic Imprinting in Epithelial and Mesenchymal Subpopulations. (A) Comparison of peaks identified and quantified by MACS analysis of H3K27ac ChIP-Seq of POS (x) and NEG (y). Peaks associated with epithelial-specific genes are labeled in blue, peaks associated with mesenchymal-specific genes are labeled in red. (B) Comparison of peaks identified and quantified by MACS analysis of BRD4 ChIP-Seq of POS $(x)$ and NEG (y). Peaks associated with epithelial-specific genes are labeled in blue, peaks associated with mesenchymal-specific genes are labeled in red. (C) Sashimi plot of H3K27ac ChIP-Seq density at the TWIST1 and ELF3 loci in POS (blue) and NEG (red). (D) Sashimi plot of BRD4 ChIP-Seq density at the TWIST1 and ELF3 loci in POS (blue) and NEG (red). (E) Log2-transformed, normalized mean RNA-Seq reads of TWIST1 and ZEB1 in NEG (red) and POS (blue). (F) Log2-transformed, normalized mean RNA-Seq reads of TWIST1 and ZEB1 in NEG (red) and POS (blue). 
subpopulations by ChIP-Seq. We used MACS to identify 12218 BRD4 peaks with a minimum of 2 reads in either the POS or NEG subpopulation, with 4169 peaks (34\%) greater than two-fold enriched in the POS subpopulation, and 2200 peaks (18\%) greater than two-fold enriched in the NEG subpopulation. We identified increased BRD4 density associated with TWIST1 and ZEB1 in the NEG subpopulation compared to the POS subpopulation, and observed similar enrichment of BRD4 density in the POS subpopulation associated with ELF3 and TP63 compared to the NEG subpopulation (Figure 10B). Focusing in further on BRD4 density at the TSS of phenotype-specific transcription factors (Figure 10D), we observe increased BRD4 density at the TSS of TWIST1 in the NEG subpopulation (red) compared to the POS subpopulation (blue), and similar enrichment of BRD4 density at the TSS of ELF3 in the POS subpopulation (blue) compared to the NEG subpopulation (red). Analysis of gene expression of the mesenchymal transcription factors TWIST1 and ZEB1 by RNA-Seq reveals significantly increased expression in the NEG subpopulation compared to the POS subpopulation (Figure 10E). Conversely, analysis of gene expression of the epithelial transcription factors TP63 and ELF3 by RNA-Seq demonstrates elevated expression of TP63 and ELF3 in the POS subpopulation compared to the NEG subpopulation (Figure 10F). These results suggest that epigenetic transcriptional activators H3K27ac and BRD4 contribute to the subpopulation-specific restriction of transcription factors that define phenotype.

\section{Drug Response Determined by Differential Remodeling of Epigenetic Landscape}

Previous studies have demonstrated that in response to the MEK inhibitor trametinib, breast cancer cells rapidly remodel their epigenetic landscape to induce a transcriptional adaptation that reactivates growth signaling of the MAPK pathway (Zawistowski et al., 2017). Therefore, to study the epigenetic factors that determine the response to an acute treatment with trametinib, we treated POS and NEG subpopulations with $30 \mathrm{nM}$ trametinib for 24 hours. This dose was chosen because it is approximately ten-fold higher than the previously published $\mathrm{IC}_{50}$ for these cells, and has been shown to be clinically achievable in patients (Infante et al., 2012). Following the acute treatment of trametinib, POS and NEG cells were fixed and stained with propidium iodide and analyzed by flow cytometry to quantify cell cycle progression (Figure 11A). Acute trametinib treatment induces a strong G1 arrest in POS cells 


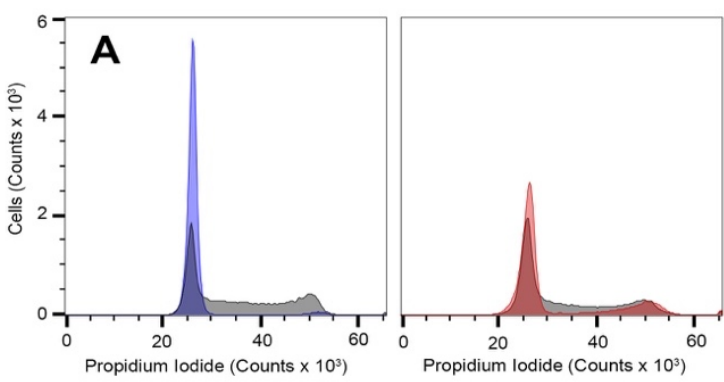

C

NEG Genes POS Genes

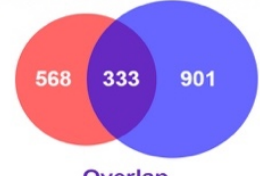

Overlap
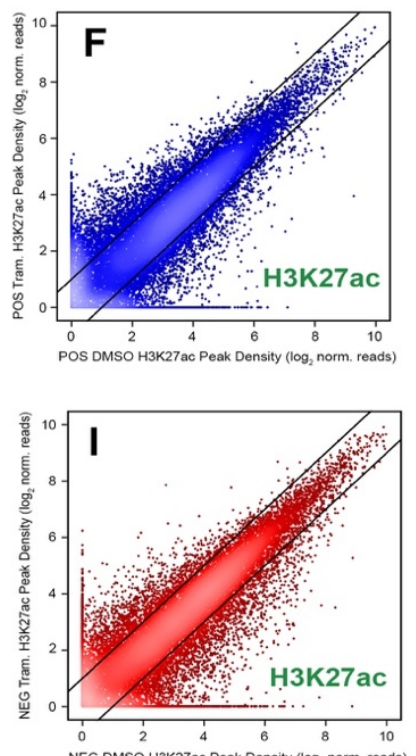

NEG DMSO H3K27ac Peak Density (log, norm. reads)

\section{D}

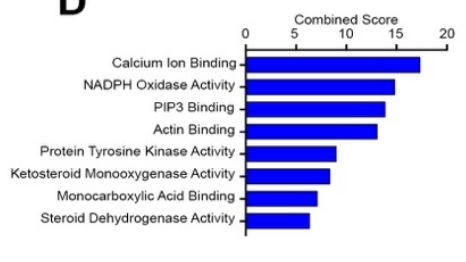

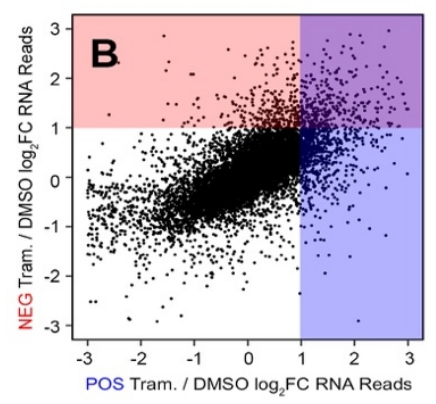

E
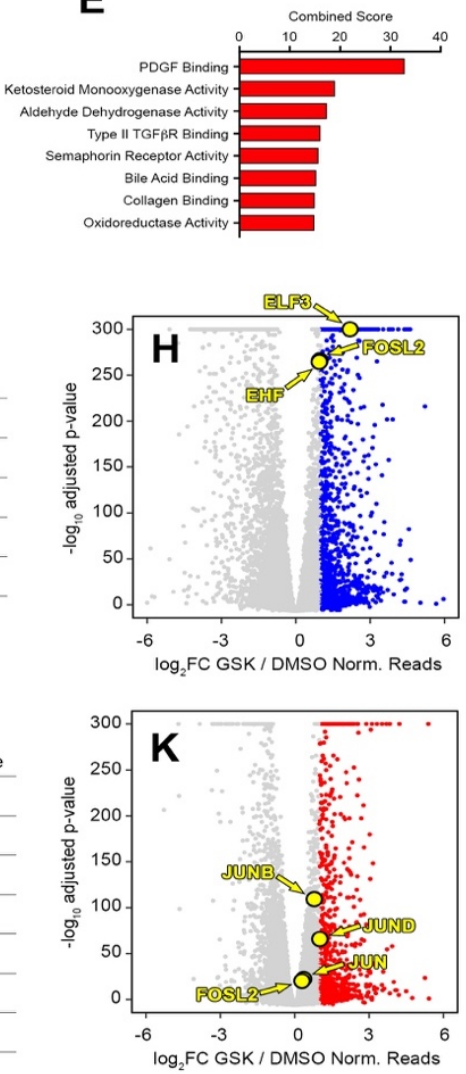

Figure 11. Differential Transcriptional and Epigenetic Response to Acute Trametinib Treatment in Epithelial and Mesenchymal Cells. (A) Cell cycle analysis of POS (blue) and NEG (red) cells following acute treatment with 30 nM trametinib for 24 hours compared to DMSO control (grey). (B) Comparison of transcriptional response in POS (x) and NEG (y) to acute treatment with 30 $\mathrm{nM}$ trametinib for 24 hours compared to DMSO control. Genes uniquely upregulated in NEG are highlighted by the red region, genes uniquely upregulated in POS are highlighted by the blue region, overlap highlighted by the purple region. (C) Venn diagram of genes that are greater than two-fold upregulated following treatment with $30 \mathrm{nM}$ trametinib treatment for 24 hours of POS and NEG. In NEG cells, 901 total genes are upregulated, 568 of which are unique. In POS cells, 1234 total genes are upregulated, 901 of which are unique. 333 genes are upregulated in both POS and NEG. (D) Gene ontology analysis of genes transcriptionally upregulated in POS cells using the Molecular Function GO terms in the Enrichr database. Combined score calculated using Enrichr database; log of p-value multiplied by z-score deviation from expected rank. (E) Gene ontology analysis of genes transcriptionally upregulated in NEG cells using the Molecular Function GO terms in the Enrichr database. Combined score calculated using Enrichr database; log of p-value multiplied by z-score deviation from expected rank. (F) Peaks identified by MACS analysis of H3K27ac ChIP-Seq of POS following treatment with $30 \mathrm{nM}$ trametinib for $24 \mathrm{hrs}(\mathrm{y})$ compared to DMSO control ( $\mathrm{x}$ ). Points $>y=x+1$ (solid line) are upregulated greater than two-fold in response to trametinib. Points $<y=(x-1)$ (solid line) are downregulated greater than two-fold in response to trametinib. (cont. on pg. 39) 
(Figure 12A, light blue) increasing G1 occupancy from $30 \%$ to $87 \%$, whereas the effect is more modest in the NEG cells, (Figure 12A, pink) increasing G1 occupancy from $41 \%$ to $67 \%$. To study the response of the POS and NEG cells to acute trametinib treatment, we exposed biological replicates of the isolated subpopulations to acute trametinib treatment, and analyzed transcription by RNA-Seq. We hypothesized that the two subpopulations would exhibit both a unique transcriptional response to trametinib and a common response to the drug, so we quantified the $\log _{2}$ fold-change in transcription following trametinib treatment relative to the DMSO control in the NEG subpopulation, and compared this response to the drug response in the POS subpopulation (Figure 11B). Genes upregulated only in the NEG subpopulation are highlighted by the red region, and genes upregulated only in the POS subpopulation are highlighted by the blue region. Genes upregulated in both the POS and NEG subpopulations are highlighted by overlap of the red and blue regions. We quantified the total genes in each region, represented by the venn diagram in Figure 11C. Interestingly, we observed the upregulation of 1234 total genes in the POS subpopulation compared to 901 total genes in the NEG subpopulation, concomitant with the stronger growth arrest in the POS subpopulation. To determine the role of upregulated genes in the drug response, we analyzed the molecular function of genes upregulated in the POS and NEG subpopulations in response to trametinib using the Enrichr gene ontology database (Chen et al., 2013; Kuleshov et al., 2016). In response to acute trametinib treatment, GO analysis identified the enrichment of calcium ion binding, PIP3 binding, actin binding and protein tyrosine kinase activity in the POS subpopulation (Figure 11D) ${ }^{5}$. Examining the underlying genes, we observe enrichment of several genes regulating the formation and binding of F-actin, such as POF1B, SYNPO2, RCSD1 and NEXN, as well as several genes controlling phospholipase activity and downstream calcium signaling, such as PLCD1, HIP1R, SYT1, and

\footnotetext{
${ }^{5}$ Figure 11. (cont.) (G) Motifs enriched at the summits of H3K27ac peaks upregulated in POS greater than two-fold and found in the promoter or enhancer region of genes transcriptionally upregulated two-fold in response to trametinib. $(\mathrm{H})$ Volcano plot of transcriptional response of POS cells to acute trametinib treatment. Log $_{2}$ fold change in gene expression in trametinib treated sample relative to DMSO control. Genes upregulated greater than two-fold highlighted in blue. Significance estimated by analysis of biological triplicate RNA-Seq reads using DESeq2. Transcription factors identified in Fig. $3 G$ highlighted in yellow. (I) Peaks identified by MACS analysis of H3K27ac ChIP-Seq of NEG following treatment with $30 \mathrm{nM}$ trametinib for 24 hrs (y) compared to DMSO control ( $x$ ). Points $>y=x+1$ (solid line) are upregulated greater than two-fold in response to trametinib. Points $<y=(x-1)$ (solid line) are downregulated greater than two-fold in response to trametinib. (J) Motifs enriched at summits of H3K27ac peaks upregulated in NEG greater than two-fold and found in the promoter or enhancer region of genes transcriptionally upregulated twofold in response to trametinib. $(K)$ Volcano plot of the transcriptional response of NEG cells to acute trametinib treatment. Log 2 fold change in gene expression in trametinib treated sample relative to DMSO control. Genes upregulated greater than two-fold highlighted in red. Significance estimated by analysis of biological triplicate RNA-Seq reads using DESeq2. Transcription factors identified in Fig. 3J highlighted in yellow.
} 

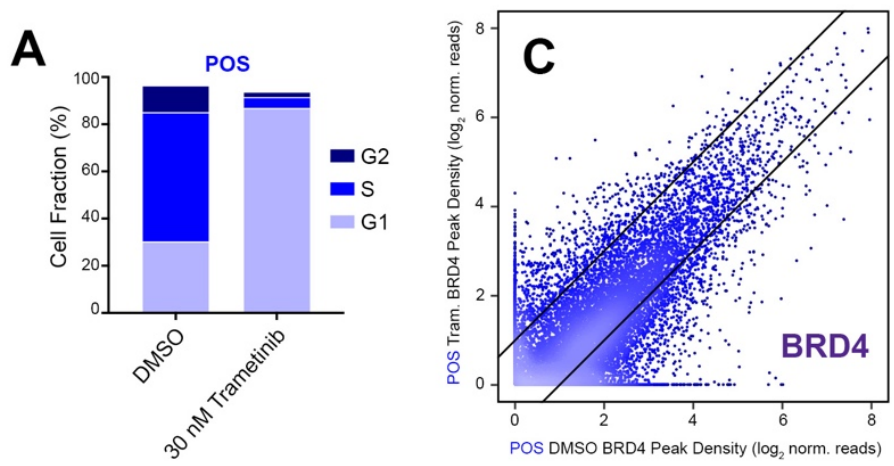

\begin{tabular}{|c|c|c|}
\hline \multicolumn{3}{|c|}{$\begin{array}{c}\text { Motifs Identified in Upregulated } \\
\text { POS BRD4 Peaks }\end{array}$} \\
\hline Motif & Name & P-value \\
\hline 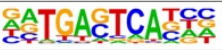 & FOSL2 & $1 \times 10^{33}$ \\
\hline TATGACTCAT & BATF & $1 \times 10^{-32}$ \\
\hline 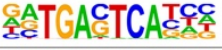 & JUN-AP1 & $1 \times 10^{31}$ \\
\hline 물술더ACTCATC & FOSL2 & $1 \times 10^{-31}$ \\
\hline 串全ATGAETCATC & FOSL1 & $1 \times 10^{-31}$ \\
\hline
\end{tabular}
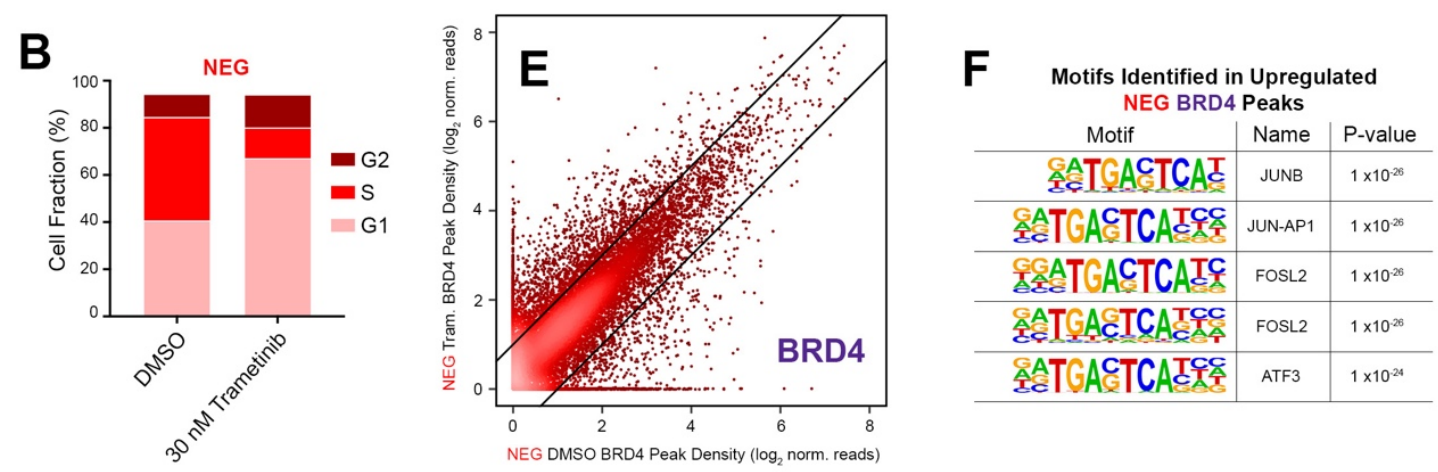

Figure 12. Supplemental Figure : Differential Transcriptional and Epigenetic Response to Acute Trametinib Treatment in Epithelial and Mesenchymal Cells. (A) Cell cycle analysis of POS cells following acute trametinib treatment with 30 nM trametinib for 24 hours. G1 (light blue), S (medium blue), G2 (dark blue) frequencies determined by curve fitting using Watson method. (B) Cell cycle analysis of NEG cells following acute trametinib treatment with $30 \mathrm{nM}$ trametinib for 24 hours. G1 (pink), S (red), G2 (dark red) frequencies determined by curve fitting using Watson method. (C) Peaks identified by MACS analysis of BRD4 ChIP-Seq of POS following treatment with $30 \mathrm{nM}$ trametinib for $24 \mathrm{hrs}(\mathrm{y})$ compared to DMSO control $(\mathrm{x})$. Points $>\mathrm{y}=\mathrm{x}+1$ (solid line) are upregulated greater than two-fold in response to trametinib. Points $<y=(x-1)$ (solid line) are downregulated greater than two-fold in response to trametinib. (D) Motifs enriched in summits of BRD4 peaks upregulated in POS greater than two-fold and found in the promoter or enhancer region of genes transcriptionally upregulated two-fold in response to trametinib. (E) Peaks identified by MACS analysis of BRD4 ChIP-Seq of NEG following treatment with $30 \mathrm{nM}$ trametinib for $24 \mathrm{hrs}(\mathrm{y})$ compared to DMSO control $(\mathrm{x})$. Points $>\mathrm{y}=\mathrm{x}+1$ (solid line) are upregulated greater than two-fold in response to trametinib. Points $<y=(x-1)$ (solid line) are downregulated greater than two-fold in response to trametinib. (F) Motifs enriched in summits of BRD4 peaks upregulated in NEG greater than two-fold and found in the promoter or enhancer region of genes transcriptionally upregulated two-fold in response to trametinib. 
ANXA8. Several of the terms identified in the calcium ion binding GO term co-occur in the PIP3 binding term, including SYT1 and ANXA8, suggesting that the upregulation is cooperative. In contrast, the NEG subpopulation is characterized by the enrichment of GO terms involved in PDGFR activation and collagen binding. Examining the underlying genes of these terms, we observe the enrichment of several collagen genes in the PDGF binding term such as COL1A1, COL3A1, COL1A2, as well as PDGFB and PDGFRB. We have previously shown that the upregulation of PDGFB and PDGFRB play a critical role in MAPK reactivation in triple negative breast cancer in response to MEK inhibitor (Duncan et al., 2012). However, we also observe the enrichment of genes involved in extracellular matrix and collagen binding, including $D D R 1, D D R 2, E C M 2$, and ITGA11. Previous reports demonstrate that epigenetic remodeling at distal regulatory elements can enhance transcriptional activation in response to targeted therapy (Stuhlmiller et al., 2015). Therefore, we performed ChIP-Seq to identify regulatory elements enriched in response to trametinib treatment and associated with transcriptionally activated genes. We identified $14063 \mathrm{H} 3 \mathrm{~K} 27 \mathrm{ac}$ peaks in the POS subpopulation with a minimum of five reads in either the trametinib-treated samples or DMSO control. Following acute trametinib treatment, we identified 2503 peaks greater than two-fold upregulated compared to the DMSO control (Figure 11F). We performed motif enrichment analysis of promoter or enhance peaks associated with upregulated genes identified by RNA-Seq using the HOMER position-weighted matrix search algorithm to identify the unique regulatory elements that may control transcriptional activation (Heinz et al., 2010). Interestingly, we discovered that the consensus motif $<$ AGGAAGT> belonging to epithelial-specific ETS-family transcription factors ELF3, ELF5 and EHF is enriched in these peaks following trametinib treatment. This search also revealed the enrichment of the consensus motif $<T G A(G / C) T C A>$ belonging to the AP-1 family members FRA1 and FRA2. We hypothesized that enrichment of the consensus sequence may correlate with increased expression of the transcription factors that bind the regulatory elements. DESeq2 analysis of the transcriptional response to trametinib revealed increased expression of ELF3, EHF and FRA2 (Figure 11H). Notably, exclusion of peaks associated with genes that are upregulated in both POS and NEG subpopulations greatly reduced the enrichment of AP-1 motifs, indicating that AP-1 may mediate a common transcriptional response to trametinib. These results suggest that increased expression of AP-1 and ETS family transcription factors can facilitate p300 recruitment to promoter and enhancer regions to mediate the transcriptional response 
to trametinib treatment. To further refine our search for distal regulatory elements associated with transcriptional activation in response to trametinib treatment, we performed ChIP-Seq for the histone acetylation reader BRD4. We identified 11240 BRD4 peaks in the POS subpopulation with a minimum of 2 reads in either the trametinib treated or DMSO control samples (Figure 12C). We observe 1333 BRD4 peaks that are upregulated at least two-fold in response to acute trametinib treatment of the POS subpopulation. Interestingly, unlike the motif searches performed on the H3K27ac ChIP-Seq, we observe an enrichment of AP-1 family motifs, including FRA2, JUNB, and FRA1, but not the epithelial-specific ETS-family transcription factors such as ELF3 and EHF (Figure 12D). In the NEG subpopulation, we identified $14456 \mathrm{H} 3 \mathrm{~K} 27$ ac peaks with a minimum of five reads in either the trametinib treated or DMSO control samples. Following acute trametinib treatment, we identified 1578 peaks greater than two-fold enriched relative to the DMSO control (Figure 11I). We performed motif enrichment analysis of promoter and enhancer peaks associated with genes transcriptionally upregulated in the NEG subpopulation in response to trametinib treatment. We identified enrichment of the $<T G A(G / C) T C A>$ characteristic of AP-1 family members, but we did not observe the enrichment of the ETS-family transcription factors that we identified in analysis of H3K27ac peaks induced in the POS subpopulation (Figure 11J). Examining the DESeq2 analysis of differentially expressed genes following trametinib treatment of the NEG subpopulation, we observe upregulation of several AP-1 family members, including JUNB, JUN, and JUND (Figure 11K). We also analyzed BRD4 binding in the NEG subpopulation in response to trametinib treatment. We identified 10970 peaks with a minimum of 2 reads in either the trametinib-treated or DMSO control samples (Figure 12E). We observed 2397 peaks were upregulated at least two-fold in the NEG subpopulation in response to trametinib treatment. We again identified the enrichment of the AP-1 family motif in BRD4 peaks upregulated in response to trametinib treatment. Previous studies have shown that the AP-1 motif is highly enriched at BRD4 binding sites, and AP-1 family members may facilitate recruitment of BRD4 to enhancer peaks to promote transcription of target genes (Najafova et al., 2017). These results suggest that recruitment of BRD4 to AP-1 binding sites following transcriptional activation of AP-1 family member may mediate the global transcriptional response to trametinib. 


\section{Differential Response to Chronic Trametinib Treatment in Isolated Subpopulations}

Recent studies have shown that the frequencies of epithelial and mesenchymal subpopulations in circulating tumor cells of breast cancer patients change in response to treatment with targeted therapy (Yu et al., 2013). Furthermore, Risom et al. demonstrated that treatment with trametinib may induce cellstate switching in basal-like breast cancer to resist the drug (Risom et al., 2018). This suggests that chronic treatment with targeted therapy applies a positive selective pressure for cells that readily adapt to the presence of the drug. Therefore, we interrogated the persistence of the acute adaptation to trametinib in the POS and NEG subpopulations during chronic treatment with $10 \mathrm{nM}$ trametinib. POS and NEG subpopulations were treated continuously with $10 \mathrm{nM}$ trametinib, and cell cycle progression was assessed by fixation followed by staining with propidium iodide and analysis by flow cytometry. The NEG subpopulation (Figure 13A) continued to proliferate during the course of chronic treatment, as indicated by the presence of the prominent G2 peak. However, similar to the acute response, the $S$ and G2 peaks are nearly completely lost following trametinib treatment of the POS subpopulation (Figure 13B). We further quantified proliferation of POS and NEG subpopulations by plating 10,000 cells per well in a 6-well dish and continuously treating those cells with $10 \mathrm{nM}$ trametinib. Cells were fixed and stained with crystal violet stain to identify drug resistant cells following treatment (Figure 13C). Increased staining at Day 21 and 49 of treatment in the NEG subpopulation indicates that these cells continue to proliferate in the presence of the drug. Quantification of crystal violet absorbance demonstrates that the NEG subpopulation continues to proliferate in the presence of trametinib (Figure 14A). In the POS subpopulation, small drug-resistant colonies appear at Day 21 of treatment. Drug-resistant colonies observed at Day 49 are considerably larger than Day 21, suggesting that these cells have adapted to proliferate in the presence of trametinib (Figure 13C). We performed RNA-Seq of the POS and NEG subpopulations to determine the persistence of the adaptive response during the course of chronic trametinib treatment. In the NEG subpopulation, we captured 12,532 genes with at least one sample with a minimum of 25 reads. We identified 1441 genes (11\%) that change at least two-fold during the course of chronic treatment (Figure 13D). We performed hierarchical clustering of differentially expressed genes and identified three distinct patterns in gene expression: genes that initially increase in response to trametinib and then return (UP-DOWN), genes that continuously decrease throughout treatment (DOWN), 



Figure 13. Persistence of Acute Response in Chronic Treatment of Subpopulations. (A) Cell cycle analysis of NEG cells following chronic treatment with $10 \mathrm{nM}$ trametinib every three days for up to 35 days beginning with Day 0 (pink) and ending with Day 35 (dark red). (B) Cell cycle analysis of POS cells following chronic treatment with $10 \mathrm{nM}$ trametinib every three days for up to 35 days beginning with Day 0 (light blue) and ending with Day 35 (dark blue). (C) Crystal violet staining of POS and NEG cells following chronic treatment with DMSO or $10 \mathrm{nM}$ trametinib every three days for up to 49 days. (D) Heatmap of gene expression in NEG cells during chronic trametinib treatment. Heatmap displays $\log _{2}$ transformed, mean-centered, normalized mean gene expression of triplicate RNA-Seq values. Genes clustered by row using average linkage of Pearson's correlation. Minimum heatmap value is white, maximum is red. Gene ontology analysis performed on each dendrogram branch using the Molecular Function GO terms in the Enrichr database. (E) Heatmap of gene expression in POS cells during chronic trametinib treatment. Heatmap displays log 2 transformed, mean-centered, normalized mean gene expression of triplicate RNA-Seq values. Genes clustered by row using average linkage of Pearson's correlation. Minimum heatmap value is white, maximum is blue. Gene ontology analysis performed on each dendrogram branch using the Molecular Function GO terms in the Enrichr database. 
A

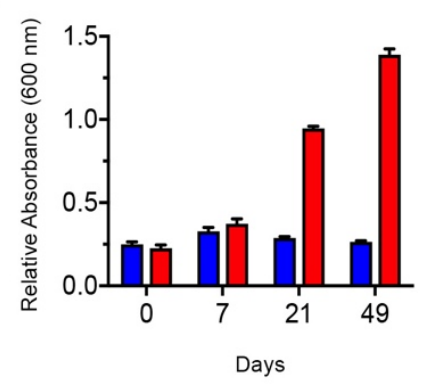

$\mathbf{E}$

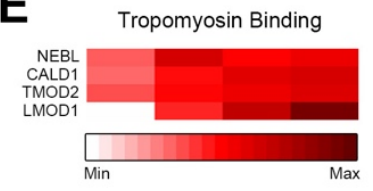

$\mathbf{F}$

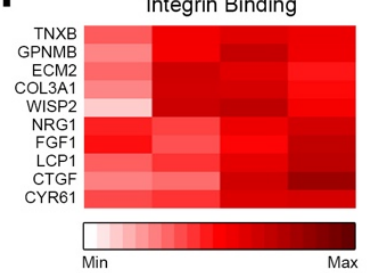

B
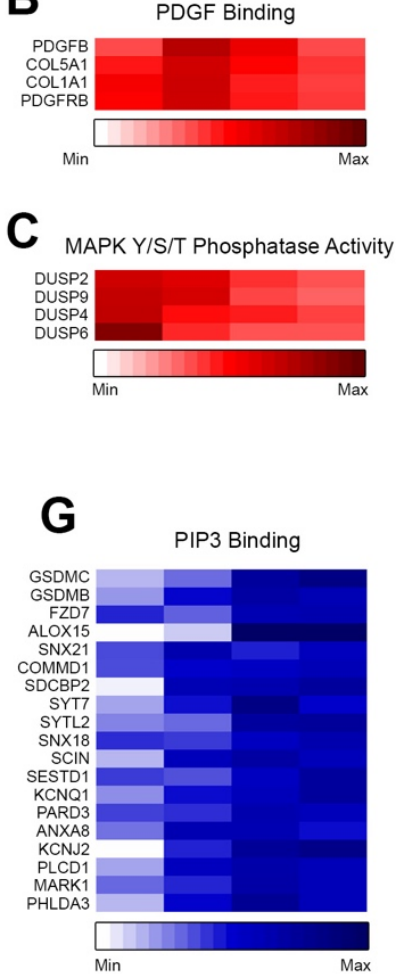

D

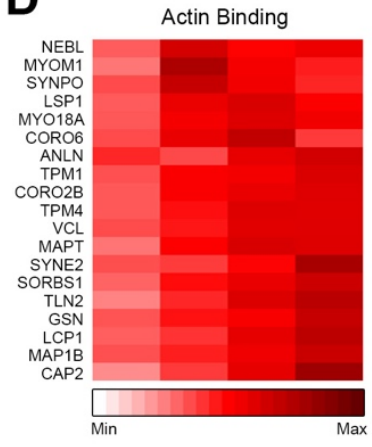

H

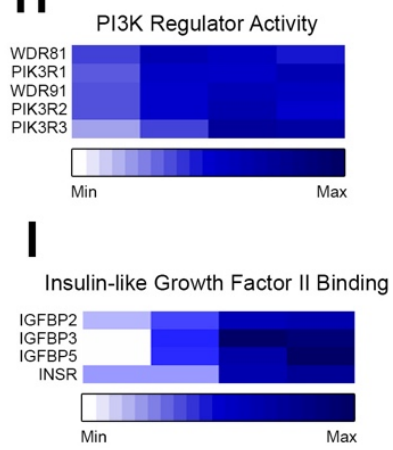

Figure 14. Supplemental Figure : Persistence of Acute Response in Chronic Treatment of Subpopulations. (A) Quantification of absorbance at $600 \mathrm{~nm}$ of the crystal violet stain dissolved in an acetic acid solution relative to the Day 0 control. (B) Heatmap of expression of genes in PDGF Binding GO term in NEG cells during chronic trametinib treatment. Heatmap displays log $_{2}$ transformed, mean-centered, normalized mean gene expression of triplicate RNA-Seq values. Minimum heatmap value is white, maximum is red. (C) Heatmap of expression of genes in MAPK Y/S/T Phosphatase GO term in NEG cells during chronic trametinib treatment. (D) Heatmap of expression of genes in Actin Binding GO term in NEG cells during chronic trametinib treatment. (E) Heatmap of expression of genes in Tropomyosin Binding GO term in NEG cells during chronic trametinib treatment. (F) Heatmap of expression of genes in Integrin Binding GO term in NEG cells during chronic trametinib treatment. (G) Heatmap of expression of genes in PIP3 Binding GO term in POS cells during chronic trametinib treatment. Heatmap displays $\log _{2}$ transformed, meancentered, normalized mean gene expression of triplicate RNA-Seq values. Minimum heatmap value is white, maximum is blue. (H) Heatmap of expression of genes in PI3K Regulator Activity GO term in POS cells during chronic trametinib treatment. (I) Heatmap of expression of genes in Insulin-like Growth Factor II Binding GO term in POS cells during chronic trametinib treatment. 
and genes that continuously increase throughout treatment (UP). We analyzed the molecular function of these gene groups by GO analysis using the Enrichr database. Interestingly, the PDGF Binding GO term identified in the acute response to trametinib is significantly enriched in the Up-Down gene cluster, suggesting that the upregulation of PDGFB, PDGFRB, COL1A, and COL5A1 is a transient response to the loss of MAPK signaling in the NEG subpopulation (Figure 14B). In the DOWN gene cluster, GO analysis reveals the enrichment of several terms related to MAPK phosphatase activity (Figure 14C), suggesting that the NEG subpopulation maintains MAPK activation in the presence of trametinib by decreasing the expression of serine-threonine phosphatase that regulate MAPK activity. In the UP gene cluster, we identified the enrichment of several GO terms that involved in the regulation of F-actin, including actin binding and tropomyosin binding, and integrin binding (Figure 14D-F). These results indicate that in response to trametinib treatment, the NEG cells reinforces focal adhesions and associated stress fibers. In contrast to the NEG subpopulation, the POS subpopulation have a stronger transcriptional response to chronic treatment with trametinib. We captured 13,118 genes with at least 25 reads, and identified 4,904 genes (37\%) that change at least two-fold in response to chronic trametinib treatment (Figure 14E). When comparing the transcriptional response of the POS and NEG subpopulations, we observe a stronger transcriptional response in the POS ( $37 \%$ of expressed genes) compared to the NEG (11\% of expressed genes). Furthermore, following hierarchical clustering of dynamic genes in the POS subpopulation, we do not observe the Up-Down gene class indicative of genes that acutely respond but return to control levels during chronic treatment. Instead, we simply observe two classes: genes that continuously decrease in expression during chronic treatment (DOWN), and genes that continuously increase in response to chronic treatment (UP). GO analysis of genes that decrease during chronic treatment show that many of these GO terms are associated with DNA replication, which does not occur in POS cells arrested in G1. POS cells also upregulate PIP3 binding and PI3K Regulator Activity (Figure 14G-H), suggesting that these genes may play a key role in mediating survival of POS cells during growth arrest with chronic trametinib treatment. Lastly, we observe the upregulation of genes regulating insulin receptor signaling, including IGFBP2, IGFBP3, IGFBP5, and INSR (Figure 14I). These data indicate decreased expression of genes regulating DNA replication occurs concomitant with G1 arrest in the POS subpopulation during chronic trametinib treatment, and survival of 
POS subpopulation is mediated by $\mathrm{PI} 3 \mathrm{~K}$ and Insulin receptor activation. In comparison, we identified a set of genes in the NEG subpopulation that acutely increase in response to trametinib but return to basal expression levels, a gene expression pattern that is not observed in the POS subpopulation. GO analysis of genes that are downregulated in the NEG during chronic trametinib treatment revealed that MAPK activation in the NEG subpopulation may be mediated by the loss of serine-threonine phosphatases.

\section{Epigenetic Remodeling Persists in Response to Chronic Trametinib Treatment}

We performed ChIP-Seq of NEG and POS subpopulations following chronic treatment with $10 \mathrm{nM}$ trametinib for two and five weeks to assess the durability of epigenetic remodeling. We identified 1017 (9\%) H3K27ac peaks upregulated in NEG cells at least two-fold at Day 14 (D14) and Day 35 (D35) out of 11659 total H3K27ac peaks in the NEG subpopulation with a minimum of five reads (Figure 15A). We performed the same analysis for BRD4 ChIP-Seq of the NEG subpopulation, and we identified 1709 (12\%) BRD4 peaks upregulated at least two-fold at D14 and D35 out of a total 14728 peaks with a minimum of two reads (Figure 15B). Notably, we observed that almost all peaks that were upregulated at least two-fold at D14 remained two-fold upregulated at D35, indicating that the epigenetic response to trametinib is imprinted within the first two weeks of treatment and persists through five weeks of treatment. To identify the regulatory elements that may be controlling the epigenetic response to trametinib during chronic treatment, we identified 105 H3K27ac peaks classified as either promoter or enhancer peaks and associated with genes that are upregulated a minimum of two-fold at D14. We performed motif analysis of these peaks, which demonstrated very weak enrichment scores (Figure 15C). However, when we performed the same motif analysis of 130 peaks we identified in BRD4 ChIP-Seq, we observed significant enrichment of AP-1 motif family members AP-1, FOSL2, JUN, FOSL1, and FOSL2 (Figure 15D). These motifs are more enriched in the chronic context compared to the acute context, suggesting that BRD4 plays an essential role in the maintenance of transcription of AP-1 targets during chronic trametinib treatment.

We performed H3K27ac ChIP-Seq following chronic treatment of POS cells with $10 \mathrm{nM}$ trametinib, and we identified 3307 (27\%) peaks upregulated at least two-fold at D14 and D35 out of a total 


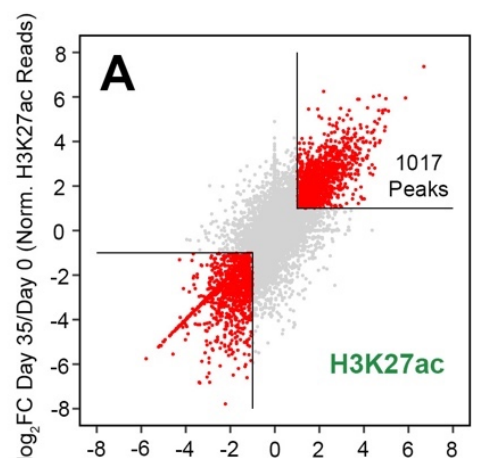

$\log _{2}$ FC Day 14/Day 0 (Norm. H3K27ac Reads)

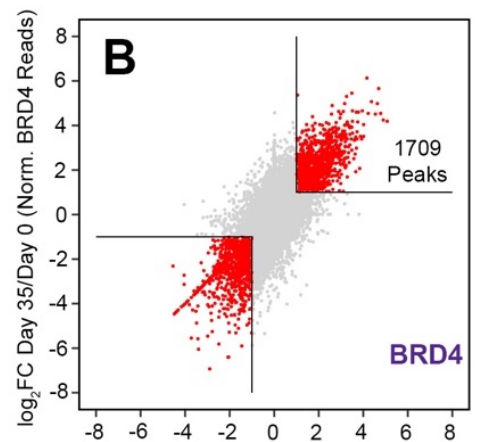

$\log _{2}$ FC Day 14/Day 0 (Norm. BRD4 Reads)
C Motifs Identified in Upregulated NEG H3K27ac Chronic Peaks

\begin{tabular}{l|l|l} 
Motif & Name & P-value
\end{tabular}

\begin{tabular}{l|l|l}
\hline CTGATGCAAC & ATF4 & $1 \times 10^{2}$ \\
\hline
\end{tabular}

TCGCAGTTE AMYB $1 \times 10^{2}$

\begin{tabular}{l|l|l}
\hline SAAACTGAAACT & IRF2 & $1 \times 10^{-2}$ \\
\hline
\end{tabular}

\begin{tabular}{l|l|l}
\hline GACCCTGGTACTCACCCTGG & ZNF322 & $1 \times 10^{-2}$ \\
\hline s.1
\end{tabular}

\begin{tabular}{l|l|l}
\hline ATTGCATCAT & CHOP & $1 \times 10^{2}$ \\
\hline
\end{tabular}

D Motifs Identified in Upregulated NEG BRD4 Chronic Peaks Motif $\mid$ Name $\quad$ P-value

\begin{tabular}{|c|c|c|}
\hline & & \\
\hline 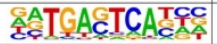 & FOSL2 & $1 \times 10^{-16}$ \\
\hline АTGACTCATC & AP-1 & $1 \times 10^{-15}$ \\
\hline 全ATGACTCATCXC & JUN/AP-1 & $1 \times 10^{-15}$ \\
\hline 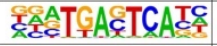 & FOSL1 & $1 \times 10^{-15}$ \\
\hline GGATGACTCATC & FOSL2 & $1 \times 10^{-15}$ \\
\hline
\end{tabular}

G Motifs Identified in Upregulated

\begin{tabular}{|c|c|c|}
\hline \multicolumn{3}{|c|}{ POS H3K27ac Peaks } \\
\hline Motif & Name & $\mathrm{P}$-value \\
\hline 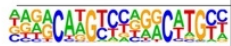 & p73 & $1 \times 10^{-15}$ \\
\hline ATGACTCATC & AP-1 & $1 \times 10^{-15}$ \\
\hline 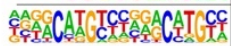 & $\mathrm{p} 63$ & $1 \times 10^{-14}$ \\
\hline 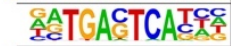 & ATF3 & $\times 10^{-13}$ \\
\hline AACATCTCCAGACATCTCCC & $\mathrm{p} 53$ & $1 \times 10^{-12}$ \\
\hline
\end{tabular}

\begin{tabular}{|c|c|c|}
\hline \multicolumn{3}{|c|}{$\begin{array}{c}\text { Motifs Identified in Upregulated } \\
\text { POS BRD4 Peaks }\end{array}$} \\
\hline Motif & Name & P-value \\
\hline 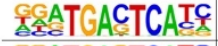 & FOSL2 & $1 \times 10^{-258}$ \\
\hline 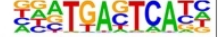 & FOSL1 & $1 \times 10^{256}$ \\
\hline 全ATGAETCATC두술 & ATF3 & $1 \times 10^{-246}$ \\
\hline 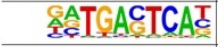 & JUNB & $1 \times 10^{232}$ \\
\hline ATGACTCATC & AP-1 & $1 \times 10^{232}$ \\
\hline
\end{tabular}

Figure 15. Epigenetic Remodeling in Response to Chronic Trametinib Treatment in Epithelial and Mesenchymal Subpopulations. (A) Comparison of H3K27ac density in NEG identified by MACS analysis of H3K27ac ChIP-Seq of NEG following chronic treatment with $10 \mathrm{nM}$ trametinib at Day $14(\mathrm{x})$ and Day $35(\mathrm{y})$ relative to Day 0. Points greater than two-fold upregulated or downregulated (solid lines) at both Day 14 and Day 35 are highlighted in red. (B) Comparison of BRD4 density in NEG identified by MACS analysis of BRD4 ChIP-Seq of NEG following chronic treatment with $10 \mathrm{nM}$ trametinib at Day 14 (x) and Day 35 (y) relative to Day 0 . Points greater than two-fold upregulated or downregulated (solid lines) at both Day 14 and Day 35 are highlighted in red. (C) Motifs enriched at H3K27ac peak summits upregulated greater than two-fold at D14 relative to Day 0 in NEG cells and found in the promoter or enhancer region of genes transcriptionally upregulated two-fold at Day 14 relative to Day 0. (D) Motifs enriched at BRD4 peak summits upregulated greater than two-fold at D14 relative to Day 0 in NEG cells and found in the promoter or enhancer region of genes transcriptionally upregulated two-fold at Day 14 relative to Day 0. (E) Comparison of H3K27ac density in POS identified by MACS analysis of H3K27ac ChIP-Seq of POS following chronic treatment with $10 \mathrm{nM}$ trametinib at Day 14 (x) and Day 35 (y) relative to Day 0. Points greater than two-fold upregulated or downregulated (solid lines) at both Day 14 and Day 35 are highlighted in blue. (F) Comparison of BRD4 density in POS identified by MACS analysis of BRD4 ChIP-Seq of POS following chronic treatment with $10 \mathrm{nM}$ trametinib at Day $14(\mathrm{x})$ and Day $35(\mathrm{y})$ relative to Day 0. Points greater than two-fold upregulated or downregulated (solid lines) at both Day 14 and Day 35 are highlighted in blue. (G) Motifs enriched at H3K27ac peak summits upregulated greater than two-fold at D14 relative to Day 0 in POS cells and found in the promoter or enhancer region of genes transcriptionally upregulated two-fold at Day 14 relative to Day 0. (H) Motifs enriched at BRD4 peak summits upregulated greater than two-fold at D14 relative to Day 0 in POS cells and found in the promoter or enhancer region of genes transcriptionally upregulated two-fold at Day 14 relative to Day 0. 
of 12290 peaks with a minimum of five reads (Figure 15E). Similar to the pattern in the NEG cells, we observed that nearly all peaks upregulated at least two-fold at D14 continued to be upregulated at D35. Interestingly, we performed motif analysis on 909 H3K27ac peaks associated with upregulated genes, and we identified several members of the p53 transcription factor family, including TP73, TP63, and TP53 in addition to AP-1 family members (Figure 15G). When considering the BRD4 peaks, we discovered that 7710 peaks $(57 \%)$, more than half of the 13613 peaks we identified with a minimum of two reads, were upregulated in response to chronic trametinib treatment. Similar to the acute response to trametinib in the POS cells, we observe a strong enrichment for the AP-1 family <TGAG/CTCA > motif in the 1723 upregulated BRD4 peaks associated with transcriptionally upregulated genes. These results indicate that AP-1 family members recruit BRD4 to promoters and enhancers of transcriptionally activated genes during chronic trametinib treatment in both the POS and NEG subpopulations, similar to the response in the acute context. However, increased H3K27ac in the POS and NEG subpopulations appears to be more specific to the cell type, with increased acetylation at loci containing the epithelial-specific transcription factor TP63.

\section{Drug Resistant POS Cells Emerge Following Chronic Trametinib Treatment}

During chronic treatment, we observed that the NEG cells continued to proliferate while the POS cells were growth arrested. However, after approximately three weeks of chronic treatment with $10 \mathrm{nM}$ trametinib, we observe small colonies of POS cells forming (Figure 13C). Therefore, we independently isolated two biological replicates of POS and NEG drug resistant cells following six weeks of treatment, and began chronic treatment with the higher dose of $30 \mathrm{nM}$ trametinib used during acute treatment. Notably, when we escalated the dose to $30 \mathrm{nM}$ trametinib, the NEG cells growth arrested but the drugresistant POS cells (POS R) continued to proliferate. We stained POS and NEG cells for senescenceassociated beta-galactosidase before and after dose escalation, and we observe prominent staining in the NEG cells that had been dose-escalated (Figure 16A, NEG R) but not the naïve cells that had never been exposed to trametinib (Figure 16A, NEG N). However, we do not observe significant staining in the drug resistant POS cells (Figure 16A, POS R) compared to the naïve cells (Figure 16A, POS N). Furthermore, we injected twelve NOD scid gamma (NSG) mice on control chow with 2 million NEG N 

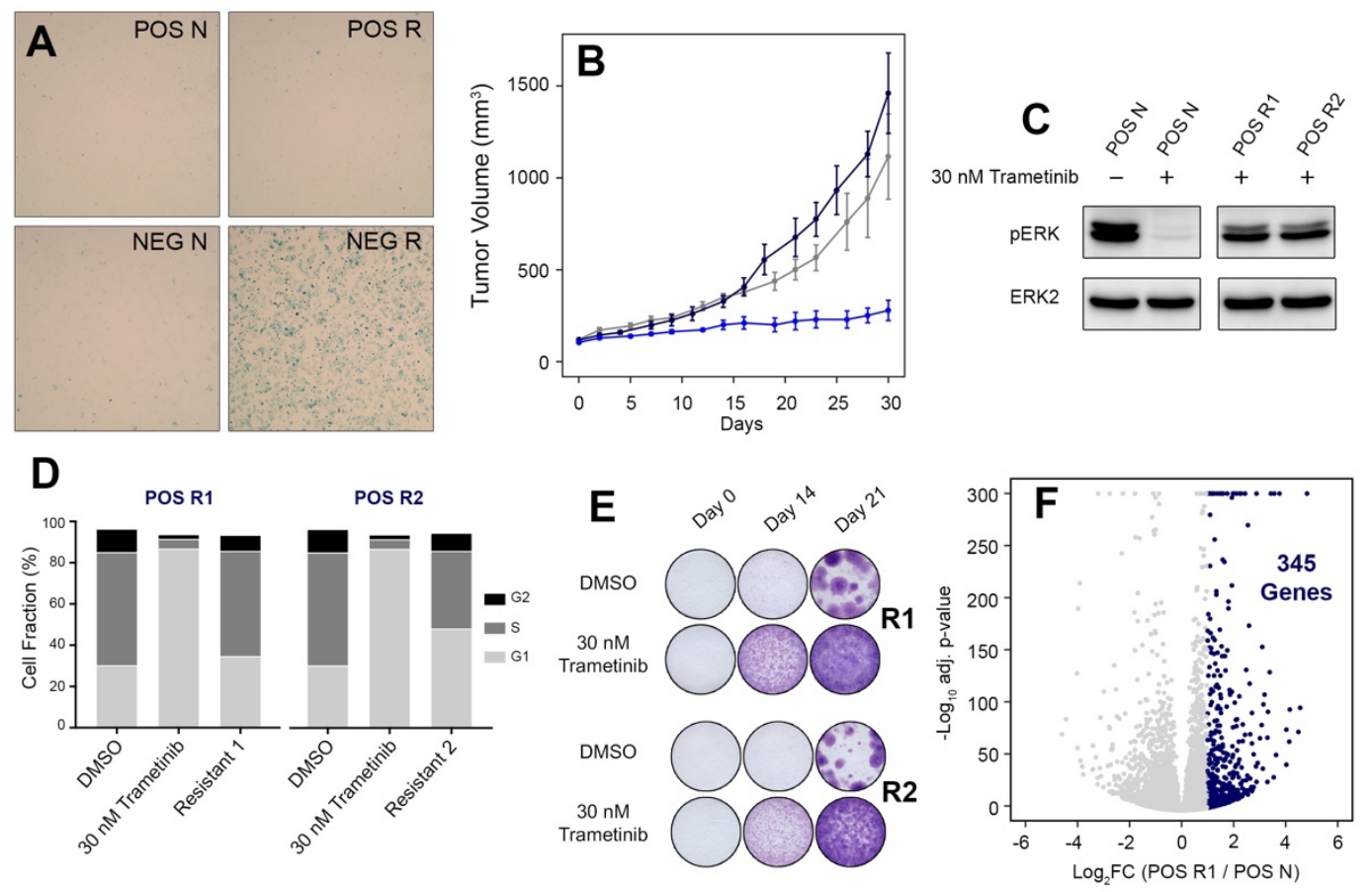

G

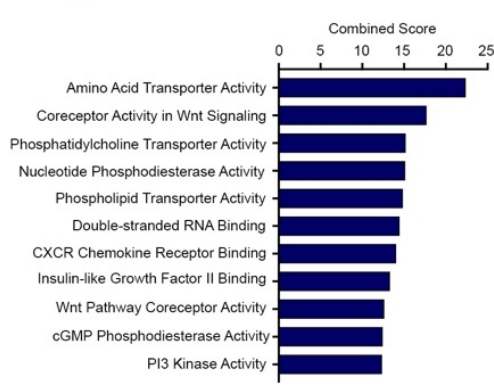

H

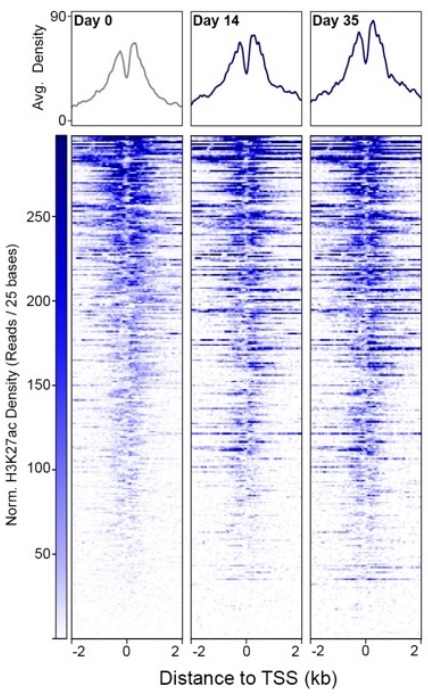

I

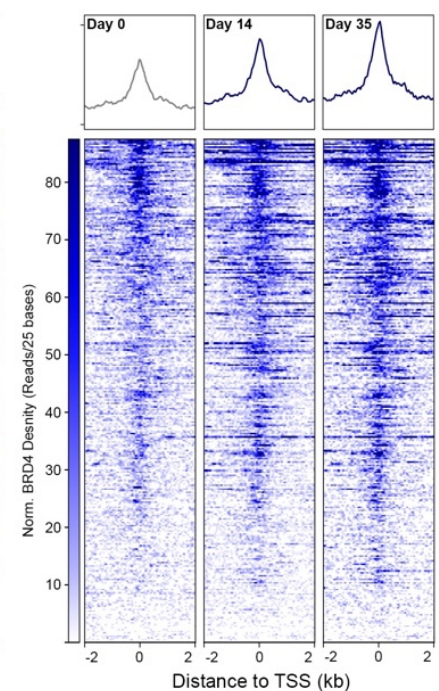

Figure 16. Resistant Cells Emerge in the Epithelial Subpopulation. (A) Beta-galactosidase staining of POS naïve (POS N) and NEG naïve (NEG N) cells before chronic treatment with $10 \mathrm{nM}$ trametinib, or beta-galactosidase staining of POS resistant (POS R) and NEG resistant (NEG R) following by dose escalation to $30 \mathrm{nM}$ trametinib after six weeks of drug conditioning with $10 \mathrm{nM}$ trametinib. (B) Tumor volume of xenograft following injection of 2 million POS naïve control (grey), POS naïve trametinib (blue) or POS resistant (navy) into the mammary fat pad of NSG mice. NSG mice injected with POS naïve cells were placed on $0.3 \mathrm{mpk} / \mathrm{day}$ trametinib chow once tumor volume reached $100 \mathrm{~mm}^{3}$. (C) Western blot for phosphorylation T202 and Y204 in the activation loop of ERK $1 / 2$ to determine activity of MEK following treatment of POS Naïve and POS resistant cells with $30 \mathrm{nM}$ trametinib. ERK2 used as loading control. (D) Cell cycle analysis of POS R1 and POS R2 cells compared to POS naïve cells following acute trametinib treatment with $30 \mathrm{nM}$ trametinib for 24 hours. G1 (light grey), S (medium grey), G2 (black) frequencies determined by curve fitting using Watson method. (E) Crystal violet staining of POS R1 and R2 following chronic treatment with DMSO control or $10 \mathrm{nM}$ trametinib every three days. (F) Volcano plot of differentially expressed genes in POS R1 cells relative to POS N cells. Log 2 fold change in gene expression in POS R1 sample relative to POS N control. Genes upregulated greater than two-fold highlighted in navy. Significance estimated by analysis of biological triplicate RNA-Seq reads using DESeq2. (cont. on pg. 51) 
cells (Figure 17A, grey) and six NSG mice on trametinib chow with 2 million NEG R cells (Figure 17A, red), and after ninety days, these mice had not developed xenograft tumors. We performed the same xenograft experiment with the POS cells, injecting twelve NSG mice on control chow with 2 million POS N cells, and six NSG mice on trametinib chow with 2 million POS R cells. Six mice in the control chow group were transferred to trametinib chow once the tumor volume reached approximately $100 \mathrm{~mm}^{3}$. POS N xenografts continued to proliferate in mice on control chow (Figure 16B, grey) ${ }^{6}$, but this proliferation was significantly reduced in POS N xenografts (Figure 16B, royal blue) in mice on trametinib chow. However, in mice on trametinib chow, the POS R xenografts (Figure 16B, navy blue) continue to proliferate at a rate similar to the POS N xenografts in mice on control chow (Figure 16B, grey). These results indicate that POS R cells have become resistant to trametinib, and continue to proliferate in presence of the drug. To determine if ERK has become reactivated in these POS R cells, we compared phosphorylation of the activation loop of ERK1/2 (T202/Y204) in the POS N treated with DMSO control or an acute dose of 30 nM trametinib for 24 hours with both the POS resistant replicates, POS R1 and POSR2, treated continuously with $30 \mathrm{nM}$ trametinib (Figure 16C). We observe that ERK phosphorylation is lost in POS N cells treated with the acute dose of trametinib compared to the DMSO control. However, ERK phosphorylation has returned in the POS R1 and POS R2 cells treated continuously with $30 \mathrm{nM}$ trametinib. We performed cell cycle analysis to determine if POS R1 and POS R2 cells are proliferating in the presence of trametinib. We observe similar frequency of cells in G1 (Figure 16D, light grey) in the POS R1 (35\%) and R2 (48\%) as the POS N cells treated with DMSO control (30\%). Furthermore, while treatment with $30 \mathrm{nM}$ trametinib induces $\mathrm{G} 1$ arrest in POS N cells, POS R1 and POS R2 cells are actively proliferating, with a similar frequency of cells in S phase in POS R1 (51\%) and POS R2 (38\%) compared to the POS N untreated control (55\%). We quantified proliferation of POS R1 and POS R2 during chronic

\footnotetext{
${ }^{6}$ Figure 16. (cont.) (G) Gene ontology analysis of genes transcriptionally upregulated in POS R1 cells using the Molecular Function GO terms in the Enrichr database. Combined score calculated using Enrichr database; log of p-value multiplied by z-score deviation from expected rank. (H) Heatmap of RSEM normalized H3K27ac reads using 5 bp bins \pm 2 kb from the TSS of 345 genes identified by DESeq analysis in Fig. 6F. White corresponds to heatmap minimum, navy corresponds to heatmap maximum. Trace displays mean density of all rows in the column, average H3K27ac density increases at Day 35 (navy) relative to Day 14 (navy) and Day 0 control (grey). (I) Heatmap of RSEM normalized BRD4 reads using $5 \mathrm{bp}$ bins $\pm 2 \mathrm{~kb}$ from the TSS of 345 genes identified by DESeq analysis in Fig. 6F. White corresponds to heatmap minimum, navy corresponds to heatmap maximum. Trace displays mean density of all rows in the column, average BRD4 density increases at Day 35 (navy) relative to Day 14 (navy) and Day 0 control (grey).
} 

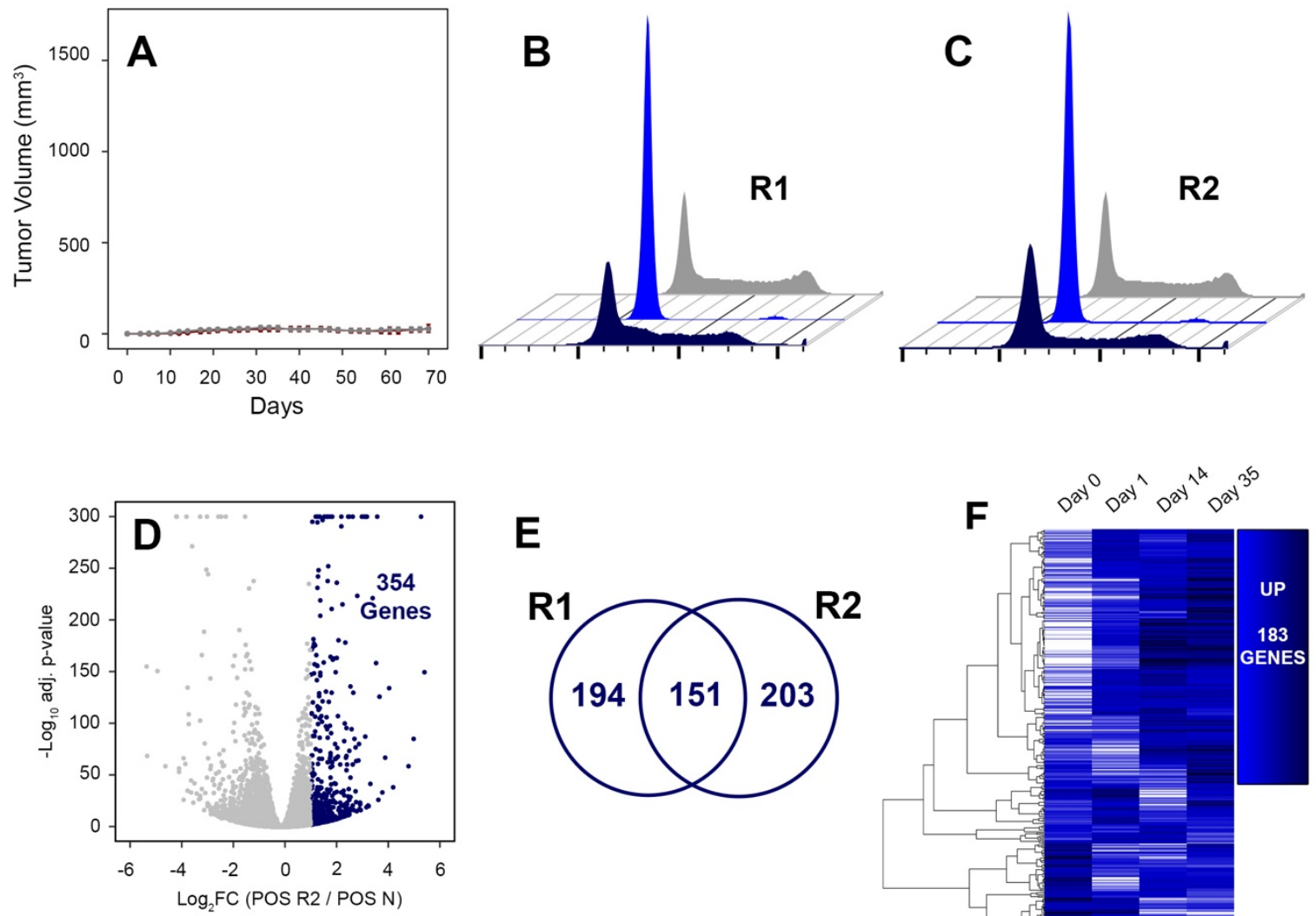

E

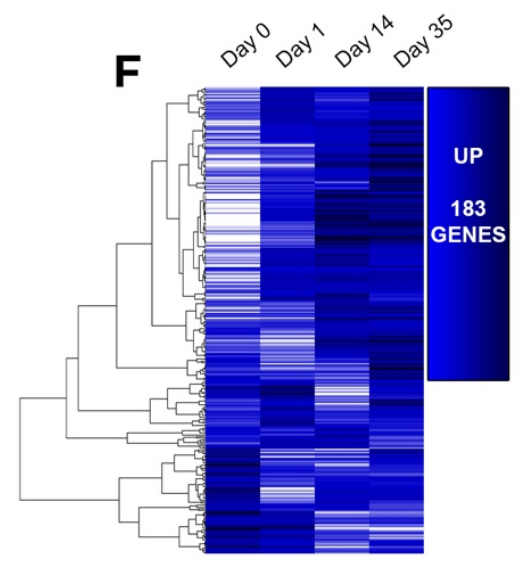

Figure 17: Supplemental Figure : Resistant Cells Emerge In Epithelial Subpopulation. (A) Tumor volume of xenograft following injection of 2 million NEG untreated control (grey) into the mammary fat pad of NSG mice on control chow and injection of 2 million NEG R following six weeks conditioning in $10 \mathrm{nM}$ trametinib into the mammary fat pad NSG mice on trametinib chow (red). (B) Cell cycle analysis of POS N following treatment with DMSO control (grey) or $30 \mathrm{nM}$ trametinib for 24 hours (blue) compared to POS R1 cells continuously cultured in $30 \mathrm{nM}$ trametinib (navy). (C) Cell cycle analysis of POS N following treatment with DMSO control (grey) or $30 \mathrm{nM}$ trametinib for 24 hours (blue) compared to POS R2 cells continuously cultured in $30 \mathrm{nM}$ trametinib (navy). (D) Volcano plot of differentially expressed genes in POS R2 cells relative to POS $\mathrm{N}$ cells. $\mathrm{Log}_{2}$ fold change in gene expression in POS R2 sample relative to POS N control. Genes upregulated greater than two-fold highlighted in navy. Significance estimated by analysis of biological triplicate RNA-Seq reads using DESeq2. (E) Venn diagram of the overlap of genes upregulated in POS R1 and POS R2 cells identified by DESeq. (F) Heatmap of genes upregulated in POS R1 cells identified by DESeq analysis, during chronic treatment of POS cells with $10 \mathrm{nM}$ trametinib. Minimum heatmap value is white, maximum is navy. 
treatment by plating cells at low density and allowing continuous cell growth for 0,14 , or 21 days (Figure 16E). We observed no crystal violet staining at Day 0 , but visible staining at Day 14 , and which intensifies at Day 21. These data confirm that the independently isolated POS R1 and POS R2 cells have developed resistance to trametinib, and are actively proliferating in the $30 \mathrm{nM}$ trametinib dose that induced growth arrest of the POS cells with acute treatment. Therefore, we performed RNA-Seq to identify genes upregulated in the POS resistant cells that contribute to trametinib resistance. DESeq analysis of genes expressed in POS R1 cells compared to POS N cells revealed 345 genes that are upregulated in POS R1 cells relative to POS N cells (Figure 16F). We analyzed the pathways activated by these 345 upregulated genes by performing GO analysis using the Molecular Function GO terms in the Enrichr database (Figure 16G). This analysis revealed enrichment of genes regulated by PI3K activity including KITLG, IRS1, NRG1, PDGFB, FGF2 and FGFR2. Furthermore, we observe increased H3K27ac and BRD4 recruitment at the PIK3R1 locus, which correlates with increased transcription during chronic trametinib treatment (data not shown). To determine the role of epigenetic remodeling associated with these activated genes, we reexamined the H3K27ac ChIP-Seq of POS cells during chronic trametinib treatment, focusing on genes upregulated in POSR1 cells. We rank-listed the 345 genes by H3K27ac density at Day 0, then created a heatmap of H3K27ac density at the transcription start site at Day 0, Day 14 and Day 35 of treatment of POS cells with $10 \mathrm{nM}$ trametinib (Figure 16H). These heatmaps reveal both an increase in H3K27ac generally, shown by increased intensity in each column, as well as specific enrichment of H3K27ac, where we can identify rows that exhibit increased H3K27ac at D14 and D35 that does not exist at Day 0. Furthermore, we quantified average H3K27ac density at Day 0 (Figure 16H, grey), Day 14 (Figure 16H, blue), and Day 35 (Figure 16H, blue), which shows an increase in average H3K27ac density during chronic trametinib treatment. We performed similar analysis of BRD4 ChIP-Seq of POS cells during chronic trametinib treatment at the 345 genes upregulated in POS R1 cells, and identified a similar pattern observed in the H3K27ac ChIP-Seq data, with increasing BRD4 density during the course of chronic trametinib treatment, as well as specific enrichment of BRD4 by row at Day 14 and Day 35 relative to Day 0 (Figure 16I). Finally, we analyzed the transcriptional pattern of resistance genes during the course of chronic trametinib treatment of POS cells (Figure 17D). Hierarchical clustering of these genes demonstrates that more than half of these genes (183 genes) continuously increase during the 
course of chronic trametinib treatment, suggesting that epigenetic remodeling associated with these genes increases transcription to drive resistance in POS cells.

\section{Acquired Resistance and Epigenetic Remodeling Contribute to Trametinib Resistance}

Several studies have demonstrated that resistance to targeted kinase inhibitors can be inherent or acquired by either adaptation or selection of resistant cells (Bhullar et al., 2018; A. Lee and Djamgoz, 2018; S. M. Miller et al., 2016; Rexer and Arteaga, 2012; Sale and Cook, 2014). In the emergence of resistant cells during chronic trametinib treatment, we can observe two modalities of resistance: the epigenetic adaptation to chronic trametinib treatment that increases expression of a gene that reactivates the MAPK pathway, as well as the selection of rare drug resistant cells during treatment. In the POSR1 DESeq, we identified 345 genes that are significantly upregulated in the resistant cells relative to untreated naïve cells. DESeq analysis identified the G-protein coupled receptor, Atypical Chemokine Receptor 3, (CXCR7) and its cognate ligand, Adrenomedullin (ADM) as highly significant genes upregulated in trametinib resistant POS R1 cells (Figure 18A), and POS R2 cells (Figure 18A). Studies have shown $\mathrm{CXCR7}$ is a $\mathrm{G}_{\mathrm{i}-\mathrm{c}}$ coupled receptor that can activate MAPK signaling via beta-arrestin to stimulate survival and proliferation in breast cancer (Rajagopal et al., 2010; Salazar et al., 2014; Sobolik et al., 2014). While RNA-Seq of POS R1 and POS R2 suggest the overexpression of CXCR7 in these cells, we confirmed the expression of CXCR7 in both POS R1 and POS R2 cells by immunoblot (Figure 18B). We reexamined the RNA-Seq data we collected during chronic trametinib treatment to determine if CXCR7 expression increases early in response to treatment, or emerges late in treatment as a result of selection. RNA-Seq analysis of CXCR7 expression demonstrates that in response to chronic trametinib treatment, CXCR7 levels increase approximately five-fold from 1173 reads to 5496 reads, and continue to increase throughout treatment to a maximum expression level of 16810 reads at Day 14 (Figure 18C). We examined ChIP-Seq for H3K27ac and BRD4 at the CXCR7 locus to determine the role epigenetic remodeling in the transcriptional activation of CXCR7. ChIP-Seq for BRD4 at the CXCR7 locus reveals increased recruitment at D14 relative to D0 (Figure 18D, light purple), which persists at D35 of treatment 

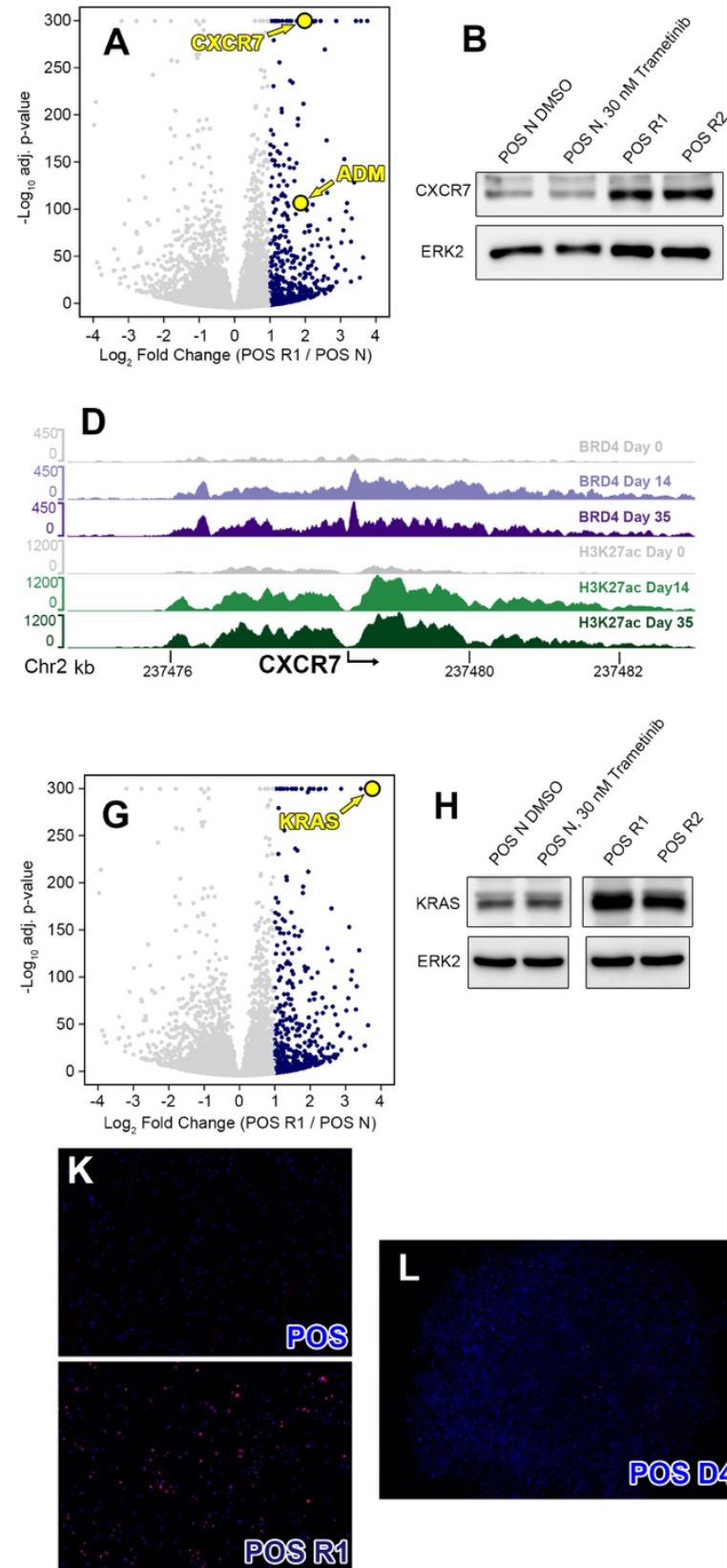
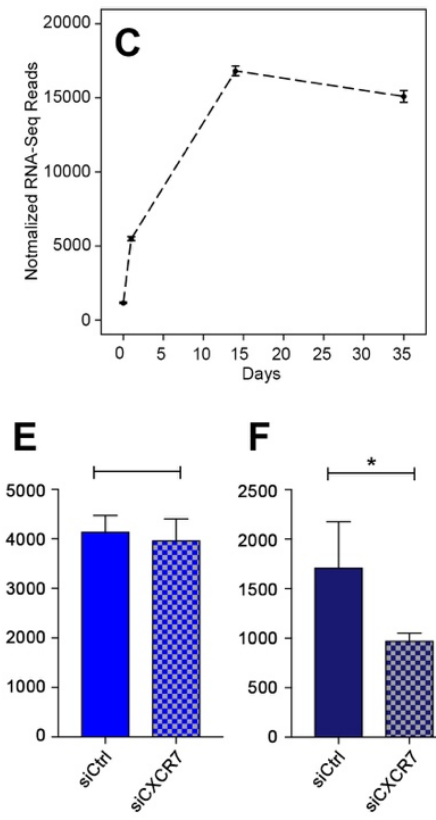

F
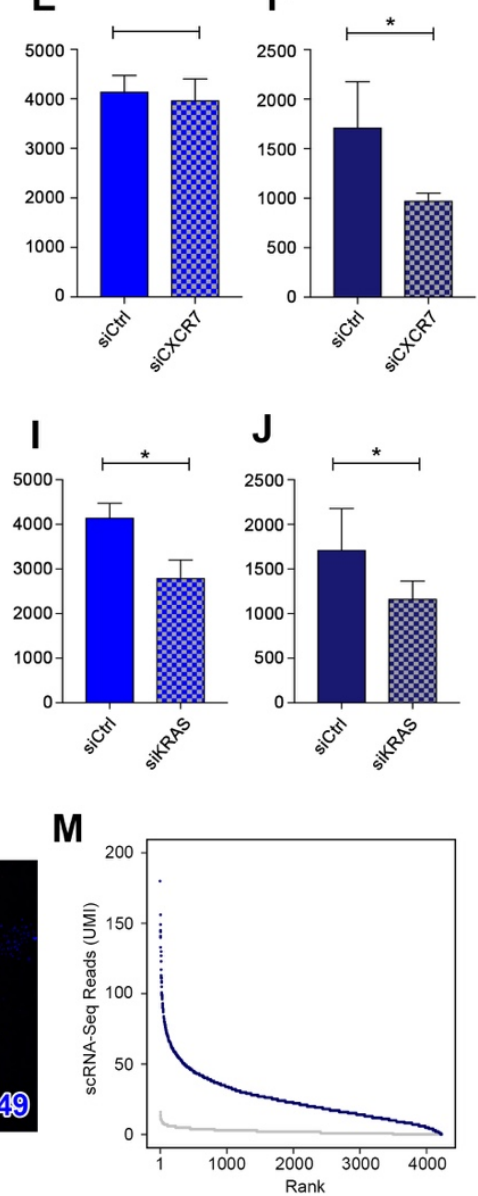

Figure 18: KRAS and CXCR7 overexpression contribute to Trametinib Resistance. (A) Volcano plot of significant genes that are differentially expressed in POS R1 (navy) relative to POS N (blue). Increased expression of the chemokine receptor CXCR7 and its cognate ligand in POS resistant are highlighted in yellow. (B) Immunoblot for CXCR7 expression in POS N following treatment with DMSO or $30 \mathrm{nM}$ trametinib for 24 hours compared to POS R1 and POS R2 continuously cultured in $30 \mathrm{nM}$ trametinib. ERK2 is used as a loading control. (C) CXCR7 expression in response to chronic treatment with $10 \mathrm{nM}$ trametinib every three days for 35 days. Gene expression is quantified by mean normalized RNA-Seq reads of three biological replicates. (D) Sashimi plot of BRD4 density at CXCR7 promoter following chronic treatment of POS cells at Day 0 (grey), Day 14 (light purple), and Day 35 (dark purple), as well as H3K27ac density at CXCR7 promoter following chronic treatment of POS cells at Day 0 (grey), Day 14 (light green), Day 35 (dark green). (E) Total cell count per well following 72 hour treatment of POS naïve with $30 \mathrm{nM}$ trametinib in combination with siRNA control (solid) or siRNA knockdown of CXCR7 (checkered). (F) Total cell count per well following 72 hour treatment of POS resistant with $30 \mathrm{nM}$ trametinib in combination with siRNA control (solid) or siRNA knockdown of CXCR7 (checkered). (G) Volcano plot of significant genes that are differentially expressed in POS R1 (navy) relative to POS N (blue). Increased expression of KRAS in POS resistant cells is highlighted in yellow. $(\mathrm{H})$ Immunoblot for KRAS in POS N following $24 \mathrm{hr}$ treatment with DMSO or $30 \mathrm{nM}$ trametinib compared to POS R1 and POS R2 continuously cultured in $30 \mathrm{nM}$ trametinib. 
(Figure 7D, dark purple). Furthermore, ChIP-Seq for H3K27ac at the CXC7 locus also demonstrates increased histone acetylation at both D14 (Figure 18D, light green) and D35 (Figure 18D, dark green). To determine the role of CXCR7 in POS R1 proliferation and survival, we quantified cell proliferation following siRNA knockdown of CXCR7 in POS N and POS R1 cells. Interestingly, CXCR7 knockdown in the POS $\mathrm{N}$ cells has no significant effect on proliferation relative to the non-targeting control (Figure 18E). However, CXCR7 knockdown in the POS R1 cells significantly decreases proliferation (Figure 18F), indicating that CXCR7 plays an essential role in mediating trametinib resistance. Additionally, DESeq analysis of POS R1 cells revealed that KRAS is significantly upregulated in trametinib resistant cells relative to the untreated control in both POS R1 (Figure 18G) and POS R2 (Figure 19B). To confirm the upregulation of KRAS in trametinib resistant cells, we performed an immunoblot for KRAS and compared KRAS expression in POS $\mathrm{N}$ treated with either DMSO or $30 \mathrm{nM}$ trametinib with KRAS expression in POS R1 and POS R2 cells (Figure 18H). These results show markedly higher expression of KRAS in both the POS R1 and POS R2 cells relative to POS N control or trametinib-treated samples. We confirmed KRAS expression in POS N and POS R1 contribute to survival and proliferation by measuring cell proliferation following treatment with siRNA targeting KRAS and a non-targeting control (Figure $18 \mathrm{I}$ and $18 \mathrm{~J})^{7}$. To determine if KRAS expression immediately increases in response to trametinib, we reexamined KRAS RNA-Seq reads at D0, D1, D14 and D35 of treatment in POS cells, but unlike CXCR7, we did not observe a substantial increase in KRAS expression during chronic treatment (Figure 19C). Therefore, we hypothesized that chronic trametinib treatment may select cells in the untreated POS population that express high levels of KRAS, and these elevated levels of KRAS mediate survival and proliferation in the presence of the drug. To determine if cells expressing high levels of KRAS exist in the untreated POS population, we performed KRAS RNA-FISH in POS N and POS R1 (Figure 18K). We observed a broad distribution of KRAS expression in the POS R1 population, with $14 \%$ of the cells classified with low

\footnotetext{
${ }^{7}$ Figure 18: (cont.) (I) Total cell count per well following 72 hour treatment of POS naïve with $30 \mathrm{nM}$ trametinib in combination with siRNA control (solid) or siRNA knockdown of KRAS (checkered). (J) Total cell count per well following 72 hour treatment of POS resistant with $30 \mathrm{nM}$ trametinib in combination with siRNA control (solid) or siRNA knockdown of KRAS (checkered). (K) RNA FISH probe targeting KRAS in POS N and POS R1 cells (red), nuclei stained with DAPI (blue). (L) RNA FISH probe targeting KRAS in POS cells (red), following chronic trametinib treated for 49 days with $10 \mathrm{nM}$ trametinib. Nuclei stained with DAPI (blue). (M) Quantification of unique transcripts captured per cell (UMI) in SCRNA-Seq. POS R1 (navy) cells are rank-listed from highest to lowest expression. POS $\mathrm{N}$ cells are rank-listed from highest to lowest expression.
} 

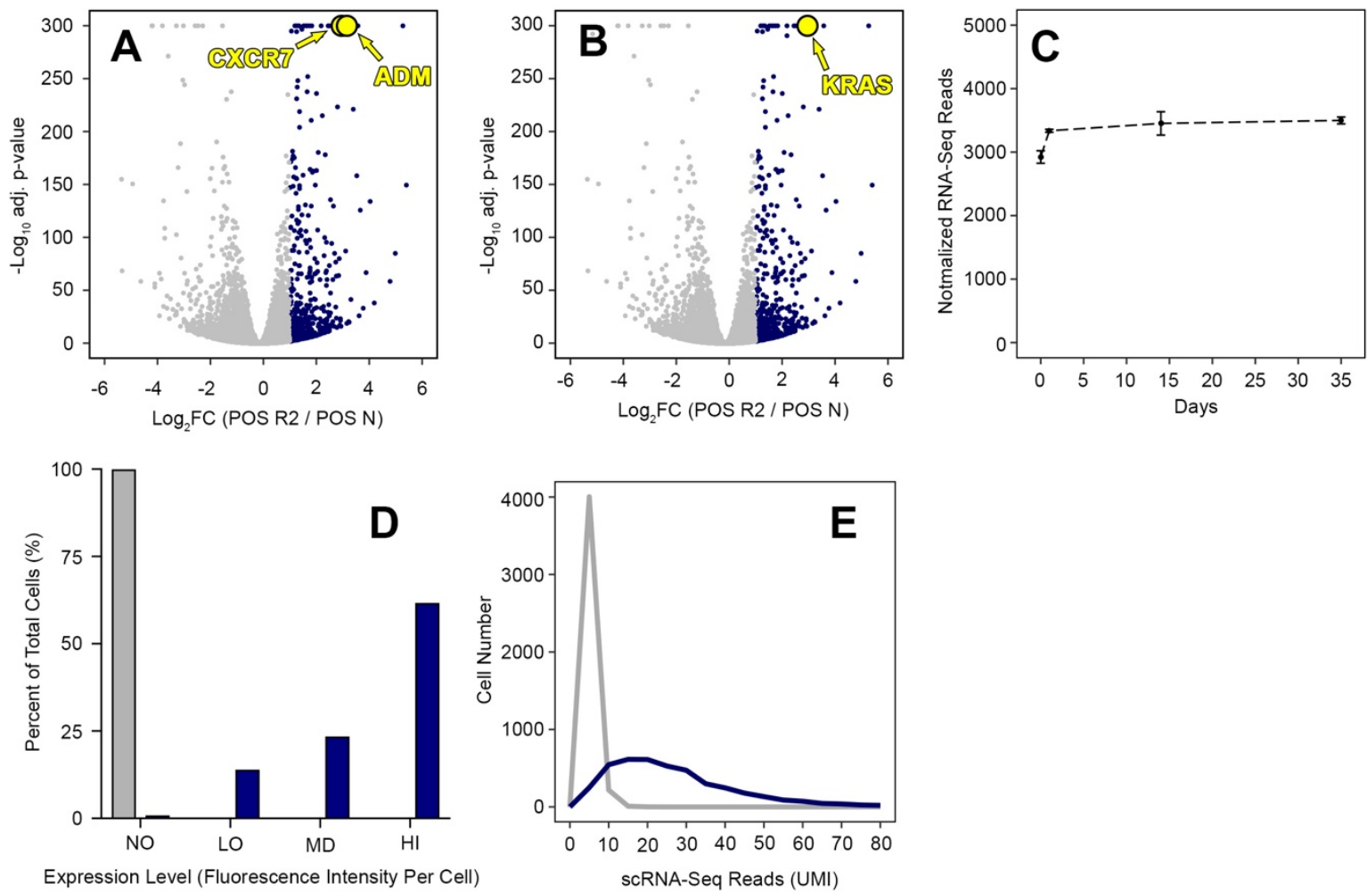

Figure 19. Supplemental Figure : KRAS and CXCR7 overexpression contribute to Trametinib Resistance. (A)Volcano plot of significant genes that are differentially expressed in POS R2 (navy) relative to POS N (blue). Increased expression of the chemokine receptor CXCR7 and its cognate ligand in POS resistant are highlighted in yellow. (B) Volcano plot of significant genes that are differentially expressed in POS R2 (navy) relative to POS N (blue). Increased expression of KRAS in POS resistant cells is highlighted in yellow. (C) KRAS expression in response to chronic treatment with $10 \mathrm{nM}$ trametinib every three days for 35 days. Gene expression is quantified by mean normalized RNA-Seq reads of three biological replicates. (D) Classification of cells in POS N (grey) and POS R1 (navy) into low, medium, and high expression groups following staining for KRAS expression using a KRAS RNA-FISH probe. (E) Distribution of KRAS expression in POS N (grey) and POS R1 (navy) cells by scRNA-Seq. Cells were sorted into bins with a range of $5 \mathrm{UMI}$ to quantify the distribution of expression in POS N and POS R1 cells. 
expression, $23 \%$ classified with medium expression, and $62 \%$ classified with high expression (Figure 19D, navy). In comparison, the untreated POS subpopulation has more uniformly low expression, with $99 \%$ of cells classified with expression below threshold (Figure 19D, grey). Interestingly, when we treated cells continuously for 35 days with $10 \mathrm{nM}$ chronic trametinib treatment, we discovered that these colonies were not significantly enriched in KRAS positive cells, suggesting this adaptation occurs after dose escalation to $30 \mathrm{nM}$ trametinib (Figure 18L). Furthermore, we performed single-cell RNA sequencing on the POS N and POS R1 cells to quantify single-cell gene expression of KRAS in these two populations. These results show a broad distribution of KRAS expression in POS R1 cells with an average of $25.2 \pm$ 18.1 reads, whereas KRAS expression in POS N cells is more uniformly low, with an average of $2.1 \pm 1.9$ reads (Figure 18M and Figure 19E). These results suggest that KRAS expression does not uniformly increase in response to trametinib treatment, and that cells that preexist in the untreated POS subpopulation are inherently more resistant to chronic trametinib treatment, and continue to proliferate in the presence of the drug. These two modalities of resistance to trametinib treatment suggest that epigenetic remodeling in response to targeted therapy can enhance transcription of genes to reactive MAPK signaling, and contribute to the proliferation and survival of cells that are inherently more resistant to the drug.

\section{Materials and Methods}

\section{Cell Culture}

Low passage SUM-229PE and isolated subpopulations were cultured in Ham's F-12 Media (Gibco cat. \# 11765-054) with 5\% fetal bovine serum (VWR cat. \# 97068-085), $5 \mu \mathrm{g} / \mathrm{mL}$ insulin (Gibco cat. \# 12585-014), 1 $\mathrm{gg} / \mathrm{mL}$ hydrocortisone (Sigma cat. \# H0396), 1\% penicillin-streptomycin solution (Gibco cat. \# 15140-014) and 5 mM HEPES (Corning cat. \# 25-060-CI). SUM-229 POS R1 and POS R2 were continuously cultured in $10 \mathrm{nM}$ trametinib for six weeks, changing the media every three days. These cells were then trypsinized and dose escalated to $30 \mathrm{nM}$ trametinib. POS R1 and POS R2 cells were continuously cultured in $30 \mathrm{nM}$ trametinib following dose escalation. 


\section{Whole Exome Sequencing Analysis}

Nextera Rapid Capture whole exome sequencing data for SUM229 EpCAM/CD49f positive or negative sorted populations was aligned to GRCh37/hg19 using BWA-MEM v 0.7.9a using -Y -M -R options and duplicates were subsequently marked using Biobambam v 0.0.172. ABRA (Mose et al., 2014) realignment was employed for indel detection and to improve variant allele frequency estimation. The resulting ABRA .bam files were piped to FreeBayes (Garrison and Marth, 2012) v 1.1.0-54 for generation of a germline variant call file whereby SUM-229 positive and negative samples were unified by nucleotide variant position. VCFtools (Danecek et al., 2011) v 0.1 .15 was employed to limit variant call file entries to Nextera Rapid Capture targeted regions. Variants were annotated using SNPEff (Cingolani et al., 2012) v 4.3t and only variants supported by a Quality score of $>30$ were considered for tabulation and plotting. Common variants or variants unique to either subpopulation were then visualized using Circos (Krzywinski et al., 2009) v. 0.69-4, where alleles for a given nucleotide position were plotted as a heatmap. Non-synonymous coding changes or frameshift changes were highlighted in a concentric ring to distinguish from synonymous coding changes and other SNPEff annotations of predicted low functional effect.

\section{Cell Lysis and Immunoblotting}

KRAS expression was detected using a mouse polyclonal anti-human KRAS antibody (Santa Cruz cat. \# sc-30). CXCR7 expression was detected using a rabbit polyclonal anti-human CXCR7 antibody (Proteintech cat. \# 20423-1-AP). ERK2 expression was detected using a rabbit polycolonal ERK2 antibody (Santa Cruz cat. \# sc-154). Rabbit primary antibodies were detected using an HRPconjugated donkey anti-rabbit secondary antibody (Jackson Laboratories cat. \# 711-035-152). Mouse primary antibodies were detected using an HRP-conjugated goat anti-mouse secondary antibody (Invitrogen cat. \# 31432). Media was aspirated and plate was washed 1X with ice-cold PBS, then plate was aspirated again. $250 \mu \mathrm{L}$ of lysis buffer (20 mM Tris-HCl, pH 8.0, 1\% NP-40, 10\% glycerol, $137 \mathrm{mM}$ $\mathrm{NaCl}, 2 \mathrm{mM}$ EDTA, $2.5 \mathrm{mM} \mathrm{NaVO}_{4}$, 1 EDTA-free protease inhibitor cocktail tablet per $25 \mathrm{~mL}$ (Roche cat. \# 11873580001), 1\% phosphatase inhibitor cocktail 2 (Sigma cat. \# P5726), and 1\% phosphatase inhibitor 3 (Sigma cat. \# P0044)) was added to plate and cells were scraped from the plate using a cell 
lifter, then lysed by vigorously pipetting cells up and down. Cell debris was pelleted at 16,000 x g, and supernatant was transferred to a new tube. Protein concentration was calculated by Bradford Assay using a bovine serum albumin standard solution. $50 \mu \mathrm{g}$ of lysate was added to each lane of $8 \%$ SDS-PAGE gel, and run at $120 \mathrm{~V}$ for approximately $2 \mathrm{hrs}$. Gel was transferred overnight to nitrocellulose membrane in transfer buffer (25 mM Tris-HCl, $192 \mathrm{mM}$ glycine, $\mathrm{pH} 8.3$ with 20\% methanol) at constant current of 180 mA. Membranes were blocked for $1 \mathrm{hr}$ with TBST (50 mM Tris- $\mathrm{HCl}, 150 \mathrm{mM} \mathrm{NaCl}, \mathrm{pH}$ 7.5, 0.1\% Tween20) $+5 \%$ powdered milk, washed three times with TBST, then incubated overnight in primary antibody diluted 1:1000 in TBST + 5\% milk. Membranes were washed three times with TBST, then incubated for 1 hr in secondary antibody diluted 1:5000 in TBST $+5 \%$ milk. Membranes were washed three times, then developed using SuperSignal Pico Plus Chemiluminescent Substrate (Thermo Fisher cat. \# 34577). Membranes were imaged using the BioRad Chemidoc Touch Imaging System.

\section{RNA Sequencing}

$10 \mathrm{~cm}$ plates were aspirated and washed with PBS, then $600 \mu \mathrm{L}$ of RPE Buffer Plus with 2mercaptoethanol was added to the plate. Cells were scraped using a cell lifter and then lysed by mechanical disruption using a 20 gauge needle. Total RNA was isolated using the RNeasy Plus Kit (Qiagen cat. \# 74134). RNA Sequencing library was prepared with $4 \mu \mathrm{g}$ of total RNA using KAPA stranded mRNA Sequencing kit (Roche cat. \# 07962193001) and Illumina TruSeq sample indexes following the manufacturer's protocol with the following exception: half of the adapter-ligated purified cDNA library was amplified with 10 cycles of PCR. The remaining library was stored at $-20^{\circ} \mathrm{C}$. Samples were pooled and sequenced as a 12-plex library pool using the 75-cycle NextSeq 500/550 High Output v2 sequencing kit (Illumina cat. \# FC-404-2005) on the Illumina NextSeq500 to yield approximately 4 x $10^{7}$ reads per sample.

\section{Chromatin Immunoprecipitation Sequencing}

ChIP-Seq for H3K27ac was performed using the ChIP-grade antibody for histone H3K27ac (Active Motif cat. \# 39133). ChIP-Seq for BRD4 was performed using the ChIP-grade antibody for BRD4 (Bethyl Laboratories cat. \# A301-985A). Fixation and immunoprecipitation were performed as previously 
described (Lovén et al., 2013; Zawistowski et al., 2017). Briefly, cells were fixed with 1\% formaldehyde for 10 minutes at room temperature, then quenched for five minutes using $1 \mathrm{mM}$ Glycine. Media was discarded and cells were scraped on ice using a cell lifter. Cells were centrifuged $3 \mathrm{~K}$ RPM at $4^{\circ} \mathrm{C}$, then supernatant was aspirated and the pellet was flash frozen in liquid nitrogen. Antibody was conjugated to Protein A Dynabeads (Life Technologies cat. \# 1002D) for 4 hours at $4^{\circ} \mathrm{C}$ in PBS $+0.5 \%$ BSA. Crosslinked pellets were resuspended in lysis buffer 1 to permeabilize plasma membrane, and centrifuged for 5 minutes at $4^{\circ} \mathrm{C}$ at $200 \mathrm{x}$ g. Nuclei were resuspended in lysis buffer 2 , and centrifuged for 5 minutes at $4^{\circ} \mathrm{C}$ at $200 \times \mathrm{g}$. Chromatin was resuspended in sonication buffer, sonication beads added and chromatin was sonicated at $4^{\circ} \mathrm{C}$ for 15 cycles; 30 seconds on, 30 seconds off. Beads were then washed three times with a low salt sonication buffer, then washed once with a high salt sonication buffer, then washed once with a $\mathrm{LiCl}$ wash buffer, then washed once with TE buffer. Fragmented chromatin was eluted from the beads with elution buffer at $65^{\circ} \mathrm{C}$ for 15 minutes, vortexing every two minutes. Eluted chromatin and Dynabeads were placed on the magnet, and eluted chromatin was transferred to a fresh tube. Crosslinking was reversed by incubating the eluted chromatin at $65^{\circ} \mathrm{C}$ overnight. The following day, $0.2 \mu \mathrm{g} / \mathrm{mL}$ RNase A (Sigma cat. \# R4642) was added to the samples and incubated for $1 \mathrm{hr}$ at $37^{\circ} \mathrm{C}$. Protein in the sample was digested using $0.2 \mu \mathrm{g} / \mathrm{mL}$ Proteinase $\mathrm{K}$ (Ambion cat. \# 25530-015) at $55^{\circ} \mathrm{C}$. Fragmented DNA was purified using the Qiagen MinElute Kit (Qiagen cat. \# 28004). 50 ng of DNA was used for library preparation using the KAPA stranded HyperPrep Kit (Roche cat. \# 07962347001) and Illumina TruSeq Indexed Adapters according to the manufacturer's instructions. Dual size selection was performed after 18 cycles of PCR amplification of the cDNA library according to manufacturer recommendations. A multiplexed pool of 12 libraries was sequenced using the 75-cycle NextSeq 500/550 High Output v2 sequencing kit (Illumina cat. \# FC-404-2005) on the Illumina NextSeq500 to yield approximately $3.5 \times 10^{7}$ reads per sample. Python scripts written for alignment and peak calling are available on Github (Singh et al., n.d.).

\section{Motif Analysis}

H3K27ac or BRD4 Peaks identified by MACS that were upregulated two-fold in response to drug treatment with a minimum of 5 reads in H3K27ac peaks in trametinib-treated sample or 2 reads in BRD4 
peaks in the trametinib treated sample were classified as upregulated peaks. Peaks $+/-5 \mathrm{~kb}$ of a gene TSS were classified as promoter peaks. Peaks located within $200 \mathrm{~kb} 5^{\prime}$ of a gene TSS or $200 \mathrm{~kb}$ 3' of a gene TES, but not located in the promoter region or gene body were classified as enhancer peaks.

Promoter and enhancer peaks that were upregulated with trametinib treatment and associated with genes that were transcriptionally upregulated greater than two-fold were classified as epigenetic activators. A $400 \mathrm{bp}$ window centered on the summits of epigenetic activator peaks was identified using the "refinepeak" option of MACS (version 2.1.2). Known consensus motifs enriched in the summits of epigenetic activator peaks were identified using the "findMotifsGenome.pl" package of Homer (version 4.10) (Heinz et al., 2010).

\section{Cell Cycle Analysis}

Approximately 1 million cells were plated in a $10 \mathrm{~cm}$ tissue culture plate, and treated for the time indicated. Treated cells were washed with PBS, trypsinized for 5 minutes, then quenched with media. Cells were pelleted at $200 \times \mathrm{g}$, and media was aspirated. Cells were resuspended in $300 \mathrm{uL} 50 \%$ FBS in PBS. Ice-cold $70 \%$ ethanol was added dropwise, and cells were fixed overnight at $4^{\circ} \mathrm{C}$. Fixed cells were pelleted at $200 \times \mathrm{g}$, then washed twice with PBS, and resuspended the fixed cells in $500 \mu \mathrm{L}$ of a propidium iodide solution (BD Biosciences cat. \# 556463). Propidium iodide staining was measured by quantifying peak area of each cell using flow cytometry running Summit flow cytometry software (version 4.3) on the Cyan ADP Flow Cytometer (Beckman Coulter). Data was normalized by adjusting the PETexRd channel voltage so that the mean of the G1 peak measured 100 counts. Cell cycle analysis was performed using FlowJo (version 10.0.8). Cells were gated to remove dead and doublet cells, then frequency of each cell cycle phase was determined using the Watson method in the cell cycle analysis package of FlowJo.

\section{Fluorescence-Activated Cell Sorting}

Approximately 50 million SUM-229PE cells were used for fluorescence-activated cell sorting (FACS). Media was aspirated from the plates and cells were washed with PBS. Cells were trypsinized for approximately 5 minutes with $0.05 \%$ Trypsin-EDTA solution (Gibco cat. \# 25300054), then resuspended 
in HF Media (Hank's Balanced Salt Solution (Gibco cat. \# 14025092) with 2\% FBS and 5 mM EDTA). Cells were resuspended in $1 \mathrm{~mL}$ of $\mathrm{HF}$ media at a concentration of $1 \times 10^{6}$ cells $/ \mathrm{mL}$ and stained with 50 uL of EpCAM (EpCAM-FITC, Stem Cell Technologies cat. \# 60136FI) and 10 uL of CD49f (CD49f-PECy5, BD Biosciences cat. \#551129) antibodies for 30 minutes in the dark at $4^{\circ} \mathrm{C}$. Cells were pelleted at $200 \mathrm{xg}$, and media was aspirated. Cells were washed twice with $10 \mathrm{~mL}$ of HF media, then filtered with a $30 \mu \mathrm{m}$ sterile cell strainer (Partec cat. \# 04-004-2326). EpCAM-positive, CD49f-positive cells and EpCAMnegative, CD49f-negative cells were sorted at single-cell purity using the FACSDiva software on the BD FACS Aria II.

\section{Crystal Violet Colony Formation Assay}

10,000 cells per well were plated in 6-well plates with three replicates for each condition at each timepoint. Media was changed every three days throughout the treatment period. Following treatment, cells were washed with PBS, fixed with ice-cold methanol for 10 minutes at $-20^{\circ} \mathrm{C}$, then stained for 20 minutes with a $20 \%$ methanol solution containing $0.5 \%$ crystal violet dye. Stain was aspirated, and wells were rinsed and dried before imaging. Stain was solubilized in $1 \mathrm{~mL}$ of $30 \%$ acetic acid solution, and absorbance of dye from each well was measured at $600 \mathrm{~nm}$.

\section{Xenograft Growth Assay}

All mice were treated in accordance with protocols approved by the Institutional Care and Use Committee for animal research at The University of North Carolina. Female NOD scid gamma mice were given a mammary fat pad injection of $2 \times 10^{6}$ POS, POS R1, NEG, or NEG R cells suspended in $50 \%$ Matrigel. Tumor volume was calculated by caliper measurements ((width) ${ }^{2} \times($ length)) / 2 every three days until tumors reached $100 \mathrm{~mm}^{3}$, at which point mice were given control chow or $0.3 \mathrm{mg} / \mathrm{kg} / \mathrm{day}$ trametinib chow. Mice injected with trametinib resistant cell lines POS R1 and NEG R were given $0.3 \mathrm{mg} / \mathrm{kg} / \mathrm{day}$ trametinib chow three days before xenograft injection and remained on trametinib chow through the duration of the study. Number of mice for each arm of the xenograft study: POS on control chow $(n=6)$, POS on trametinib chow $(n=6)$, NEG on control chow $(n=12)$, POS R1 on trametinib chow $(n=6)$, NEG R 
on trametinib chow $(n=6)$. Tumor volume of NEG and NEG R tumors did not reach the $100 \mathrm{~mm}^{3}$ minimum volume, and after 90 days the NEG and NEG R arms of the study were terminated.

\section{Beta-Galactosidase Staining}

Cells were stained using Senescence $\beta$-Galactosidase Staining Kit (Cell Signaling Technology cat. \# 9860) according to the manufacturer's instructions. Briefly, cells were cultured in a $35 \mathrm{~mm}$ cell culture dish. Media was aspirated and cells were washed with PBS. Cells were fixed for 10-15 minutes at room temperature using $1 \mathrm{~mL}$ of the $1 \mathrm{X}$ Fixative Solution prepared as recommended. Plate was rinsed twice with PBS, then $1 \mathrm{~mL}$ of the $\beta$-Galactosidase Staining Solution was added to the plate, which was sealed with parafilm and incubated overnight at $37^{\circ} \mathrm{C}$ in a dry incubator. Plates were stored in $70 \%$ glycerol at $4^{\circ} \mathrm{C}$.

\section{siRNA Transfection}

Individual siRNA from siGENOME smartpools targeting CXCR7 (GE Dharmacon cat. \# MQ013212-03-0002) and KRAS (GE Dharmacon cat. \# MQ-005069-00-0002) were resuspended in siRNA buffer (GE Dharmacon cat. \# B-002000-UB-100). Cells were transfected using RNAiMAX (Invitrogen cat. \# 13778-075) with 25 nM siRNA. siGENOME non-targeting pool \#2 was used as control siRNA (GE Dharmacon cat. \# D-001206-14-05). Cells were incubated for 48 hours to ensure target knockdown, and RNA was isolated using the RNeasy Plus RNA isolation kit (Qiagen cat. \# 74134). RNA was converted to cDNA using the High Capacity cDNA Reverse Transcription kit (Applied Biosystems cat. \# 4368813), then target expression was assessed by quantitative real-time PCR using taqman probes targeting KRAS (Hs00364284_g1) and CXCR7 (Thermofisher cat. \# Hs00664172_s1) compared to ACTB control (Thermofisher cat. \# Hs01060665_g1) and Taqman Fast Universal PCR Master Mix (Thermofisher cat. \# 4352042). The siRNA with the best results was selected for future studies.

\section{Cell Proliferation Assay}

Cells were plated in a 96-well plate and transfected with $25 \mathrm{nM}$ of siRNA targeting KRAS or CXCR7. Cells were incubated for 24 hours following transfection, then media was aspirated. Cells were 
treated with $30 \mathrm{nM}$ trametinib for 72 hours, then stained with Hoescht 33342 at $2.5 \mu \mathrm{g} / \mathrm{mL}$ for 30 minutes at $37^{\circ} \mathrm{C}$. Plates were imaged using a Thermo Cellomics ArrayScan VTI, capturing 25 frames per well. Cell number was quantified and siRNA target was compared siRNA control.

\section{RNAscope detection of KRAS RNA}

KRAS RNA was detected using the RNAscope ${ }^{\circledR} 2.5$ LS Probe for Hs-KRAS-O1 (ACD Biotechne cat. \# 522968) in a Bond RX autostainer (Leica Biosystems) following the manufacturer's directions. TSACy5 and DAPI were used to visualize RNAScope signal and nuclei, respectively.

\section{Scanning and analysis of IF images}

Slides containing RNAscope fluorescently labeled cells were scanned either in the Aperio ScanScope FL or the Aperio Versa Digital Pathology Scanner using a 20X objective (Leica Biosystems). Images were archived in TPL's eSlide Manger database (Leica Biosystems). Images were manually annotated for regions of interest using Tissue Studio software (Definiens Inc.Tissue Studio version 2.7 with Tissue Studio Library version 4.4.2), specifically the Nuclei and Simulated Cells algorithm in the IF Portal, was then used to detect and enumerate cells that contained the cy5-labeled KRAS RNA. Briefly, nuclei were digitally detected by the presence of DAPI stain (nuclear counterstain). From these nuclei, a cell simulation was performed, and cells margins were grown out from nuclear boundaries. Positivity thresholds for KRAS RNA probe were determined by measuring the staining intensities both inside and outside simulated cells. Once thresholds were set, the algorithm evaluated each cell individually for the presence of KRAS RNA positive signal. Cells were separated by the algorithm into negative, low positive, medium positive, or high positive categories based on the signal intensity. $\mathrm{H}$-Score values were determined using the following formula: $[1 \times(\%$ cells $1+)]+[2 \times(\%$ cells $2+)]+[3 \times(\%$ cells $3+)]$.

\section{Single Cell RNA Sequencing}

Single cell RNA Sequencing of approximately 5000 POS cells and 5000 POS R1 cells was performed according to the manufacturer specification (10X Genomics cat. \# 1000092, 1000074, 120262) Briefly, media was aspirated and cells were washed with PBS, then trypsinized with $0.05 \%$ Trypsin for 5 
minutes. Media was added to stop digestion and cells were centrifuged at $200 \times \mathrm{g}$ for 5 minutes. Cells were resuspended in $1 \mathrm{~mL}$ of media, then filtered with a $30 \mu \mathrm{m}$ filter and counted. Cells were washed twice with $0.04 \%$ BSA, then strained using a FlowMi tip strainer (Sigma cat. \# BAH136800040) to minimize sample loss. Single cell libraries were prepared according to the manufacturer's instructions. Library concentration was calculated by Agilent Tapestation, and library was amplified using 8 cycles of PCR according to manufacturer's recommendation. Library was sequenced using the 150-cycle NextSeq 500/550 High Output v2.5 sequencing kit (Illumina cat. \# 20024907) on an Illumina NextSeq 500 Sequencer. The cellranger mkfastq pipeline of 10x Genomics Cell Ranger v. 2.1.0 software was utilized to demultiplex 3' Chromium v2 sequence reads. The resulting FASTQ files were piped to cellranger count for alignment, filtering, barcode counting, and UMI counting to generate the gene-barcode matrix for single-cell KRAS expression estimates of each SUM-229 subpopulation.

\section{Discussion}

A pathological partial response in triple negative breast cancer patients can be caused by tumor heterogeneity resulting from chromosomal instability or epigenetic plasticity that allows cell switching to a drug tolerant state. In this study, we use the epithelial and mesenchymal subpopulations of a cell line isolated from a TNBC patient to examine the role of cell state in epigenetic remodeling following treatment with the MEK inhibitor trametinib. Analysis of the whole exome sequencing of the POS and NEG subpopulations using a germline variant caller highlights the genetic similarity of these two subpopulations, suggesting that these two phenotypes are epigenetically regulated. Indeed, a previous study using these POS and NEG subpopulations demonstrated that siRNA knockdown of SMARCD3, a regulatory subunit of the SWI/SNF chromatin remodeling complex, is sufficient to induce a switch from the NEG to POS phenotype (Jordan et al., 2013). SMARCD3 knockdown in the NEG subpopulation induces loss of SNAIL and SLUG, indicating that the SWI/SNF chromatin remodeling complex plays an important role in maintaining the expression of these mesenchymal-specific transcription factors. The role of epigenetic regulation of cell identity can be observed in the enrichment of H3K27ac and BRD4 peaks associated with transcription factors TWIST1 and ZEB1 in the NEG subpopulation that maintain the mesenchymal phenotype, and TP63 and ELF3 in the POS subpopulation that maintain the epithelial 
phenotype. Previous studies have shown that knockdown of ZEB1 is sufficient to induce expression of ECadherin and desmoplakin concomitant with loss of mesenchymal markers SNAIL1 and SNAIL2 (An et al., 2017). An analogous study demonstrated that knockdown of TP63 is sufficient to induce an epithelial to mesenchymal transition in breast cancer cells, demonstrating the role of TP63 in the maintenance of the epithelial phenotype (Lindsay et al., 2011). RNA-Seq analysis of POS and NEG isolated subpopulations revealed elevated expression of MET in both the POS and NEG subpopulations, which had been observed in previous studies of SUM-229 PE cells. However, we also observed subpopulation specific expression of tyrosine kinases PDGFR $\alpha$ and AXL in the NEG subpopulation, whereas we observed specific expression of EGFR and SRC in the POS subpopulation. The differential expression of these tyrosine kinases likely governs the proliferation of these two subpopulations, as the POS subpopulation is more proliferative but more sensitive to trametinib, whereas the NEG subpopulation is less proliferative but also less sensitive to trametinib.

Cancer cells can rapidly remodel chromatin in response to targeted therapy to enhance transcription of genes that mediate drug resistance. Analysis of the transcriptional response in a panel of breast cancer cell lines demonstrated that while some genes were commonly upregulated in all cell lines, some genes were specifically upregulated in mesenchymal or epithelial cell lines (Zawistowski et al., 2017). In the context of a heterogenous tumor, these distinct transcriptional responses in epithelial and mesenchymal cells can have a profound impact on outcome, as these unique responses may not be discretely targeted by monotherapy. Therefore, we used the isolated subpopulations of the heterogenous triple negative breast cancer cell line SUM-229 PE to identify the unique regulatory elements that control the epigenetic response to acute trametinib treatment. Interestingly, motif analysis of upregulated H3K27ac promoter or enhancer peaks associated with genes upregulated in the POS subpopulation identified several epithelial-specific ETS-family transcription factor motifs. It is well established that MAP kinases can directly phosphorylate ETS-family transcription factors, which act as effectors of MAPK signaling (Plotnik et al., 2014; Selvaraj et al., 2015). Furthermore, biochemical studies of ETS-family transcription factors demonstrated that the histone acetyltransferase p300 preferentially binds to phosphorylated ETS-1 and ETS-2, suggesting that these transcription factors play an essential role in remodeling $\mathrm{H} 3 \mathrm{~K} 27 \mathrm{ac}$ in response to targeted therapy in epithelial cells by recruiting p300 to target genes 
(Foulds et al., 2004). Strikingly, motif analysis of the upregulated BRD4 promoter and enhancer peaks revealed enrichment of only AP-1 family motifs $<$ TGAG/CTCA $>$ in the most highly enriched sequences. Genome-wide analysis of BRD4 binding has demonstrated that this transcriptional coactivator specifically localizes with AP-1 family members FOSL2 and JUND at enhancers (Najafova et al., 2017). These results are supported by the finding that BRD4 binds direct to C-Jun (S.-Y. Wu et al., 2013). Furthermore, siRNA knockdown of either FOSL2 or JUND decreases BRD4 binding at these enhancers and decreases transcription of target genes. This indicates that AP-1 family members can recruit BRD4 to enhancers to stimulate transcription of target genes and increase survival in the presence of the drug. Surprisingly, motif analysis of the H3K27ac peaks in the NEG subpopulation did not reveal any motifs unique to this subpopulation, but did identify the enrichment of the AP-1 family motif. This suggests that several factors beyond DNA sequence can control epigenetic remodeling in response to targeted therapy, such as chromatin accessibility and structure. Similar to the motif enrichment of BRD4 peaks in the POS subpopulation, analysis of upregulated BRD4 peaks associated with genes upregulated in the NEG subpopulation revealed that the most highly enriched motifs belong to AP-1 family members. This indicates that BRD4 can be recruited by AP-1 family members in either the POS or NEG subpopulation to stimulate gene transcription in response to trametinib treatment, suggesting a universal role for BRD4 in growth signaling.

While acute drug treatments offer a tractable approach to study the response to targeted therapy, this approach cannot capture the chronic adaptation to continued therapy that may occur during a clinical treatment regimen. Therefore, we treated POS and NEG subpopulations continuously during a five week treatment window to determine how cells adapt to chronic treatment. Similar to the acute response to trametinib, we observed that the NEG cells continued to proliferate in the presence of trametinib in both cell cycle analysis and crystal violet assays. GO analysis of the transcriptional response to chronic treatment with trametinib demonstrates that NEG cells upregulate TGF $\beta$ signaling, which can activate MAPK signaling in mesenchymal cells via PAK2 in a SMAD-independent manner (Suzuki et al., 2007; Wilkes et al., 2005). These results indicate that the NEG cells can stimulate MAPK signaling by activation of autocrine signaling of the TGF $\beta$ receptor, a pathway that is unable to be activated in the POS epithelial cells. Activation of MAPK signaling in NEG cells is further stimulated by the downregulation of dual 
specificity serine-threonine phosphatases that dephosphorylate MAP kinases and negatively regulate pathway activation. The loss of negative regulation of MAPK pathway activity, combined with activation of MAPK by stimulation of autocrine TGF $\beta$ signaling induces pathway reactivation during chronic trametinib treatment and allows NEG cells to continue to proliferate in the presence of the drug. In response to chronic trametinib treatment, cell cycle analysis and crystal violet assays demonstrate that most POS cells growth arrest. This is also reflected in the transcriptional response to chronic trametinib treatment, where we observe the downregulation of GO terms controlling DNA replication, including multiple terms related to DNA helicase activity. However, these cells promote survival by increasing transcription of the regulatory subunit of PI3K and PIP3 binding proteins, which stimulates PI3K signaling during chronic trametinib treatment.

Motif analysis of BRD4 peaks upregulated in response to chronic trametinib treatment revealed a similar enrichment compared to the acute trametinib response in both POS and NEG subpopulations, which is characterized by the specific enrichment of AP-1 family members. Furthermore, motif enrichment analysis of H3K27ac peaks upregulated in NEG cells during chronic trametinib treatment revealed few highly significant motifs, likely the result of many fewer H3K27ac peaks upregulated in the NEG cells compared the POS cells (1017 peaks compared to 3307 peaks, respectively). Interestingly, analysis of the upregulated H3K27ac peaks in POS cells identified the TP63 consensus sequence as one of the most highly enriched motifs. During chronic trametinib treatment, TP63 expression peaks at Day 14 before returning near basal expression levels at Day 35, suggesting that TP63 expression may play a role in promoting survival during chronic trametinib treatment. Indeed, TP63 has been shown to promote survival in mammary epithelial cells by increasing transcription of cell adhesion genes including ITGB1, ITGB4, and ITGA6, which stimulates anti-apoptotic focal adhesion signaling (Carroll et al., 2006). We observe increased transcription of ITGB4 concomitant with TP63 expression at Day 14, which return near basal expression levels at Day 35.

Drug resistant cells can emerge from a low-frequency subpopulation of slowly proliferating persister cells that survive treatment and adapt to the therapy. Hata et al. demonstrated that cells initially resistant to targeted therapy can develop secondary genetic alterations that increase proliferation in the presence of the drug (Hata et al., 2016). We used two independently isolated trametinib-resistant cell 
lines to identify key genes that drive the survival and proliferation of drug tolerant persister cells in triple negative breast cancer. DESeq analysis demonstrated the chemokine receptor, CXCR7, and its cognate ligand, adrenomedullin (ADM) are significantly upregulated in both POS R1 and POS R2 cells. Results from RNA sequencing following acute treatment with trametinib shows that these genes are significantly upregulated after 24 hours of treatment with $30 \mathrm{nM}$ trametinib. Furthermore, RNA sequencing during chronic treatment with $10 \mathrm{nM}$ trametinib demonstrates that CXCR7 expression increases nearly fifteenfold from a baseline expression level of approximately 1200 reads at Day 0 , to a peak expression level of 17000 reads at Day 14. Additionally, increased H3K27ac and BRD4 binding can be observed at the CXCR7 TSS, suggesting that epigenetic remodeling at the CXCR7 locus contributes to transcriptional activation. siRNA knockdown of CXCR7 had no effect in the POS naïve cells, but reduced proliferation in POS R1 cells, suggesting that CXCR7 plays an essential role in MAPK activation in drug resistant cells. Furthermore, CXCR7 overexpression is correlated with metastatic breast cancer and is associated with decreased overall survival and metastatic-free survival in breast cancer patients. Analysis of tumor gene expression from 525 invasive ductal carcinoma patients in the TCGA database showed that $16 \%$ of patients have CXCR7 overexpression, and these patients have significantly worse overall survival compared to the normal expression cohort (Wani et al., 2014). Analysis of tissue microarray cores following immunohistochemical staining for CXCR in 10 normal, 10 metastatic and 38 invasive ductal carcinoma samples shows a significant correlation between CXCR7 expression and aggressive breast cancer (Wani et al., 2014). These data indicate that CXCR7 overexpression is associated with poor prognosis and survival, and may contribute to resistance to treatment with trametinib. DESeq analysis of POS resistant cells compared to the untreated control cells also identified the upregulation of KRAS in trametinib resistant cells. Gene expression analysis of breast cancer patients in the TCGA database demonstrates that KRAS overexpression is also clinically observed (Cancer Genome Atlas Network, 2012). siRNA knockdown of KRAS in both the untreated control and POS resistant cells reduces proliferation compared to the control siRNA. Furthermore, quantification of RNA-FISH staining of untreated control cells demonstrates low KRAS expression, but we observe a wider distribution KRAS levels in drug resistant cells, suggesting that the increased expression of KRAS contributes to fitness in presence of trametinib. Single cell RNA sequencing of the POS naïve and POS R1 confirms the broad 
distribution of KRAS expression in the drug resistant cells, as well as subset of cells in the POS R1 population and POS N population that have similar expression levels. These results indicate that KRAS overexpression in the drug resistant cells contributes to proliferation in the presence of trametinib, but the broad distribution of expression suggests that there may be other factors, including expression of CXCR7 that contribute to survival.

Epigenetic remodeling in response to targeted therapy has been shown to promote the survival of persister cells and contribute to drug resistance. Therefore, understanding the mechanisms of epigenetic remodeling in response to targeted therapy is key to developing durable therapies for triple negative breast cancer patients. Here, we identify unique signaling and epigenetic adaptations to trametinib in the mesenchymal NEG cells and epithelial POS cells. MAPK pathway reactivation by downregulation of dual specificity phosphatases, as well as upregulation of TGF $\beta$ signaling can maintain proliferation of NEG cells in the presence of trametinib. However, epithelial-specific ETS family members ELF3 and EHF may play a key role in the recruitment of the histone acetyltransferase p300 at transcriptionally activated genes in the POS cells, which increases transcription of CXCR7 to reactivate MAPK in POS cells. These results indicate that trametinib monotherapy may be insufficient to a induce pathological complete response in triple negative breast cancer, but could be combined with epigenetic inhibitors to achieve a more durable response. 


\section{CHAPTER 5: CONCLUDING REMARKS}

Intratumor heterogeneity represents a significant obstacle in the development of durable therapies for the treatment of triple negative breast cancer. Gene expression profiling of breast cancer has improved the accuracy of patient prognosis and identified novel therapies for each molecular subtype, but heterogeneity can confound prognosis and limit the efficacy of individual agents. Single cell gene expression analysis demonstrates that triple negative tumors often contain several distinct subpopulations. These subpopulations can display a spectrum of phenotypes and demonstrate differential sensitivity to targeted therapy. Furthermore, chronic treatment with kinase inhibitors can induce the selection of a drug resistant subpopulation (Shah et al., 2012; Yu et al., 2013). Indeed, a comparison of the pretreatment biopsy and post-resection tumors of breast cancer patients treated with either docetaxel or letrozole revealed that the molecular subtype of the tumor can change during treatment (Creighton et al., 2009). Therefore, it is important to understand the mechanisms of resistance to targeted therapy in each subpopulation to better design combination therapies that improve treatment durability and block resistance.

In this work, we identified genetically similar epithelial and mesenchymal subpopulations that demonstrate differential localization of epigenetic activators at the promoter and enhancer regions of transcription factors known to maintain cell identity. RNA sequencing of the two isolated subpopulations following acute trametinib treatment revealed genes that were commonly upregulated in response to the drug, as well as genes that were uniquely upregulated in response to the drug. We hypothesized that epigenetic remodeling in response to trametinib may activate the transcription of genes involved in MAPK reactivation and cell survival. Therefore, we identified H3K27ac and BRD4 peaks found in the promoter or enhancer regions of upregulated genes, and performed motif searches to identify transcription factors that mediate the drug response in epithelial and mesenchymal cells. We identified the enrichment of epithelialspecific ETS family transcription factors ELF3 and EHF in POS cells, which were not observed in NEG cells. Furthermore, we identified increased transcription of ELF3 and EHF in POS cells in response to 
trametinib treatment. Increased transcription of ELF3 and EHF may increase binding at the promoter and enhancer regions associated with ETS-regulated genes to recruit p300 and increase H3K27ac (Figure 20). Therefore, we will perform ChIP-Seq for these transcription factors to quantify recruitment of ELF3 and EHF at promoter and enhancer regions of transcriptionally activated genes following acute trametinib treatment. Furthermore, I will perform RNA sequencing of POS cells treated with trametinib or DMSO control following transfection with siRNA targeting ELF3 or EHF and identify genes that are transcriptionally activated in response to trametinib that are sensitive to loss of ELF3 or EHF. Motif analysis also revealed that the AP-1 motif was universally enriched at BRD4 peaks upregulated in response to trametinib treatment. Interestingly, BRD4 has been found to regulate the expression of AP-1 family members FOSL2 and JUND, and is enriched at AP-1 binding sites (Najafova et al., 2017). This suggests that BRD4 recruitment can stimulate transcription of AP-1 targets to reactivate MAPK signaling and inhibition of BRD4 may block this transcriptional activation. However, further bioinformatics analysis of BRD4 peaks sensitive to bromodomain inhibitors would be required to test this hypothesis.

Chronic trametinib treatment demonstrates that epithelial cells remain sensitive to chronic treatment while the mesenchymal cells continue to proliferate in the presence of the drug. Analysis of gene expression in POS and NEG cells during chronic trametinib demonstrates that in NEG cells, a class of genes acutely respond to trametinib treatment, whereas in POS cells this acute response is not observed. We identified the upregulation of TGF $\beta$ and TGF $\beta$ R in response to trametinib treatment, which may reactivate MAPK signaling in the presence of trametinib. To test this hypothesis, I will perform cell cycle analysis of NEG cells following treatment with trametinib alone or in combination with the TGF $\beta R$ inhibitor galunisertib. Interestingly, when we examined chromatin remodeling in response to chronic treatment in POS and NEG cells, we found that peaks upregulated at Day 14 of treatment remain upregulated at Day 35 (Figure $15 \mathrm{~A}, \mathrm{~B}, \mathrm{E}$, and F). These results indicate that chromatin remodeling in response to trametinib persists throughout chronic treatment and targeting chromatin remodeling may synergize with trametinib treatment for therapeutic benefit. Enrichment of BRD4 at AP-1 binding sites in both POS and NEG cells suggests that BRD4 inhibitors may be able to block chromatin remodeling and transcriptional upregulation of AP-1 target genes. Previous studies have demonstrated that combining the bromodomain inhibitor JQ1 with trametinib abrogates transcriptional upregulation of RTKs in breast 
POS
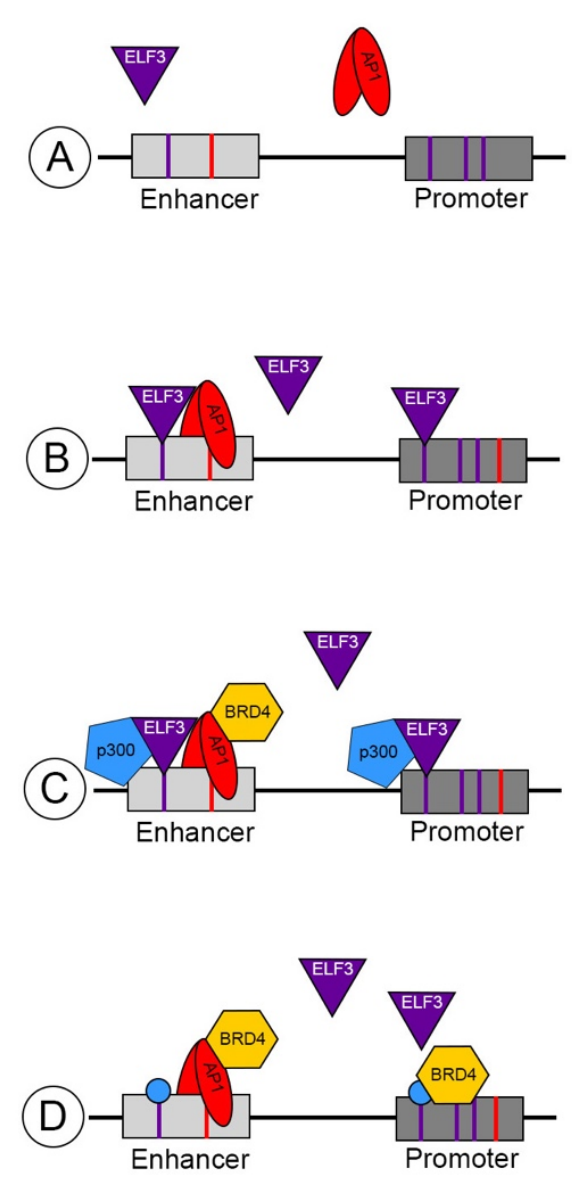
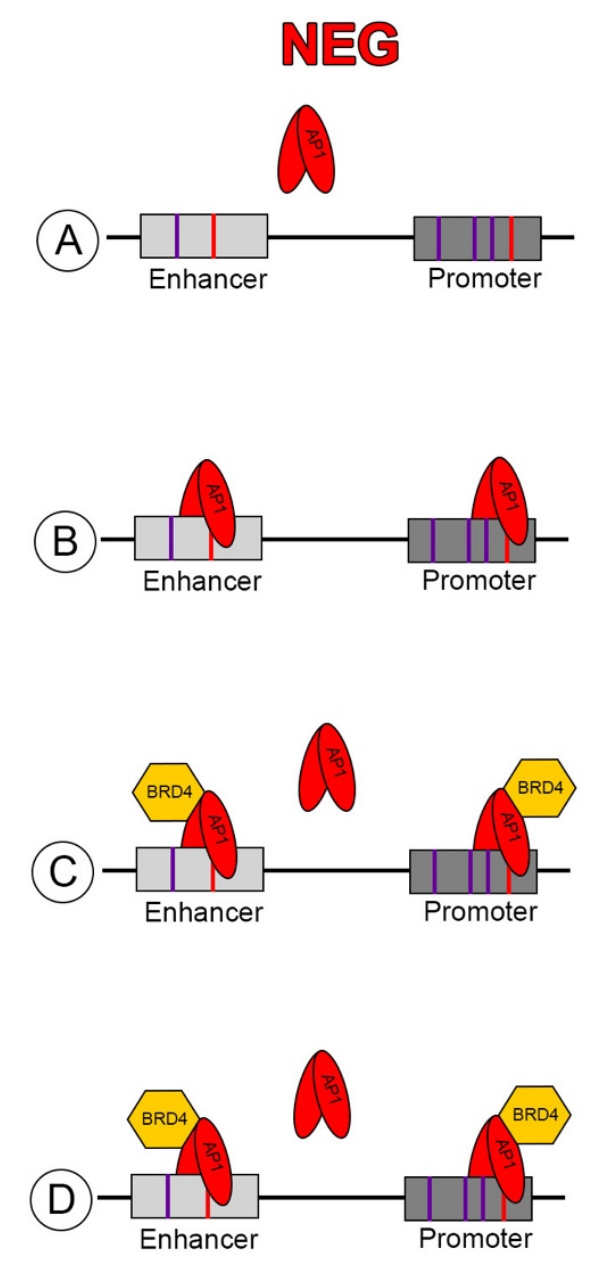

Figure 20. Epigenetic Remodeling in Response to Trametinib Treatment in Subpopulations of Triple Negative Breast Cancer. (A) ELF3 (purple triangle) is expressed in POS but not NEG cells and can bind to ELF3 motif (purple line), whereas AP1 (red ellipse) is expressed in both POS and NEG subpopulations. (B) Treatment with trametinib increases ELF3 (purple triangle) expression in the POS subpopulation and expression of AP1 (red ellipse) in both the POS and NEG subpopulations. (C) Increased binding of ELF3 (purple triangle) recruits p300 (blue pentagon), whereas AP1 (red ellipse) binding in both the POS and NEG subpopulations recruits BRD4 in response to trametinib treatment. (D) Increased recruitment of p300 deposits H3K27ac (blue circle), and increased AP1 and $\mathrm{H} 3 \mathrm{~K} 27 \mathrm{ac}$ recruits BRD4 to transcriptionally activate genes. 
cancer, and slows the emergence of drug resistant cells (Stuhlmiller et al., 2015; Zawistowski et al., 2017). Future studies may focus on the role of BRD4 recruitment to AP-1 sites and the effectiveness of epigenetic inhibitors to block trametinib resistance in POS and NEG cells. BET bromodomains have demonstrated utility both as single agents and in combination with kinase inhibitors, and clinical studies are currently being organized to test these therapies in breast cancer patients.

Chromatin remodeling in response to targeted therapy can enhance transcriptional upregulation of genes that promote survival and increase drug resistance. We observe the enrichment of H3K27ac and BRD4 binding at the promoter and enhancer regions associated with CXCR7, which is correlated with increased transcription of the target gene. Knockdown of CXCR7 in POS resistant cells demonstrates that upregulation of CXCR7 contributes to survival and proliferation of these cells. Studies of CXCR7 signaling

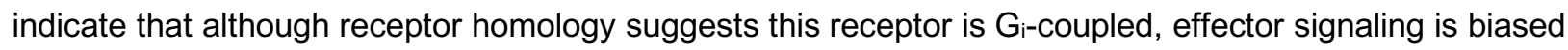
to $\beta$-arrestin (Rajagopal et al., 2010; Salazar et al., 2014). Additionally, treatment of POS resistant cells with the $G_{i}$ inhibitor pertussis toxin has no effect on proliferation of these cells, further suggesting that CXCR7 signaling is biased to $\beta$-arrestin. Unfortunately, genetic and chemical inhibitors of $\beta$-arrestin signaling are limited, which prevents further elucidation of the role of CXCR7 in MAPK activation. Currently, there are no commercially available inhibitors of CXCR7, but given the findings in this study, as well as the established role in the regulation of proliferation and metastasis, development of CXCR7 inhibitors may be warranted.

The development of kinase inhibitors for the treatment of cancer has revealed that intratumor heterogeneity and epigenetic plasticity remain significant obstacles for the development of durable therapies. Preclinical studies have demonstrated that drug tolerant persister cells emerge during treatment with targeted therapy as a result intratumor heterogeneity (Hata et al., 2016). Similar work in breast cancer demonstrated that drug treatment can induce epigenetic remodeling in persister cells and increase resistance (Risom et al., 2018). Furthermore, our work has demonstrated that epithelial and mesenchymal subpopulations can have distinct responses to targeted therapy, which differentially mediate drug resistance in these cells. These results highlight the need for novel drug combinations that prevent epigenetic remodeling and transcriptional activation in response to targeted therapy. Therefore, continued work to understand the mechanism of epigenetic remodeling and concomitant transcriptional 
activation in response to therapy is paramount to developing synergistic combination therapies for the treatment of cancer. 


\section{REFERENCES}

Alver, B.H., Kim, K.H., Lu, P., Wang, X., Manchester, H.E., Wang, W., Haswell, J.R., Park, P.J., Roberts, C.W.M., 2017. The SWI/SNF chromatin remodelling complex is required for maintenance of lineage specific enhancers. Nat Commun 8, 14648. doi:10.1038/ncomms14648

An, J., Zheng, Y., Dann, C.T., 2017. Mesenchymal to Epithelial Transition Mediated by CDH1 Promotes Spontaneous Reprogramming of Male Germline Stem Cells to Pluripotency. Stem Cell Reports 8, 446-459. doi:10.1016/j.stemcr.2016.12.006

Andre, F., O'Regan, R., Ozguroglu, M., Toi, M., Xu, B., Jerusalem, G., Masuda, N., Wilks, S., Arena, F., Isaacs, C., Yap, Y.-S., Papai, Z., Lang, I., Armstrong, A., Lerzo, G., White, M., Shen, K., Litton, J., Chen, D., Zhang, Y., Ali, S., Taran, T., Gianni, L., 2014. Everolimus for women with trastuzumabresistant, HER2-positive, advanced breast cancer (BOLERO-3): a randomised, double-blind, placebo-controlled phase 3 trial. The Lancet Oncology 15, 580-591. doi:10.1016/S14702045(14)70138-X

Avivar-Valderas, A., Wen, H.C., Aguirre-Ghiso, J.A., 2014. Stress signaling and the shaping of the mammary tissue in development and cancer. Oncogene 33, 5483-5490. doi:10.1038/onc.2013.554

Bachelot, T., Bourgier, C., Cropet, C., Ray-Coquard, I., Ferrero, J.-M., Freyer, G., Abadie-Lacourtoisie, S., Eymard, J.-C., Debled, M., Spaëth, D., Legouffe, E., Allouache, D., Kouri, El, C., Pujade-Lauraine, E., 2012. Randomized phase II trial of Everolimus in combination with Tamoxifen in patients with hormone receptor-positive, human epidermal growth factor receptor 2-negative metastatic breast cancer with prior exposure to aromatase anhibitors: A GINECO study. J. Clin. Oncol. 30, 2718-2724. doi:10.1200/JCO.2011.39.0708

Badve, S., Turbin, D., Thorat, M.A., Morimiya, A., Nielsen, T.O., Perou, C.M., Dunn, S., Huntsman, D.G., Nakshatri, H., 2007. FOXA1 expression in breast cancer--correlation with luminal subtype $A$ and survival. Clin. Cancer Res. 13, 4415-4421. doi:10.1158/1078-0432.CCR-07-0122

Baselga, J., Gelmon, K.A., Verma, S., Wardley, A., Conte, P., Miles, D., Bianchi, G., Cortés, J., McNally, V.A., Ross, G.A., Fumoleau, P., Gianni, L., 2010. Phase II trial of Pertuzumab and Trastuzumab in patients with human epidermal growth factor receptor 2-positive metastatic breast cancer that progressed during prior trastuzumab therapy. J. Clin. Oncol. 28, 1138-1144.

doi:10.1200/JCO.2009.24.2024

Belinsky, S.A., Nikula, K.J., Palmisano, W.A., Michels, R., Saccomanno, G., Gabrielson, E., Baylin, S.B., Herman, J.G., 1998. Aberrant methylation of p16INK4a is an early event in lung cancer and a potential biomarker for early diagnosis. Proc Natl Acad Sci USA 95, 11891-11896.

doi:10.1073/pnas.95.20.11891

Bhullar, K.S., Lagarón, N.O., McGowan, E.M., Parmar, I., Jha, A., Hubbard, B.P., Rupasinghe, H.P.V., 2018. Kinase-targeted cancer therapies: progress, challenges and future directions. Molecular Cancer 17, 48. doi:10.1186/s12943-018-0804-2 
Biswas, S., Rao, C.M., 2017. Epigenetics in cancer: Fundamentals and Beyond. Pharmacology and Therapeutics 173, 118-134. doi:10.1016/j.pharmthera.2017.02.011

Blackwell, K.L., Burstein, H.J., Storniolo, A.M., Rugo, H.S., Sledge, G., Aktan, G., Ellis, C., Florance, A., Vukelja, S., Bischoff, J., Baselga, J., O'Shaughnessy, J., 2012. Overall survival benefit with Lapatinib in combination with Trastuzumab for patients with human epidermal growth factor receptor 2-positive metastatic breast cancer: Final results from the EGF104900 study. J. Clin. Oncol. 30, 2585-2592. doi:10.1200/JCO.2011.35.6725

Blick, T., Widodo, E., Hugo, H., Waltham, M., Lenburg, M.E., Neve, R.M., Thompson, E.W., 2008. Epithelial mesenchymal transition traits in human breast cancer cell lines. Clin Exp Metastasis 25, 629-642. doi:10.1007/s10585-008-9170-6

Brown, J.D., Lin, C.Y., Duan, Q., Griffin, G., Federation, A., Paranal, R.M., Bair, S., Newton, G., Lichtman, A., Kung, A., Yang, T., Wang, H., Luscinskas, F.W., Croce, K., Bradner, J.E., Plutzky, J., 2014. NF$\mathrm{KB}$ directs dynamic super enhancer formation in inflammation and atherogenesis. Molecular Cell 56, 219-231. doi:10.1016/j.molcel.2014.08.024

Brown, R., Curry, E., Magnani, L., Wilhelm-Benartzi, C.S., Borley, J., 2014. Poised epigenetic states and acquired drug resistance in cancer. Nature Genet 14, 747-753. doi:10.1038/nrc3819

Cancer Genome Atlas Network, 2012. Comprehensive molecular portraits of human breast tumours. Nature 490, 61-70. doi:10.1038/nature11412

Capelan, M., Pugliano, L., De Azambuja, E., Bozovic, I., Saini, K.S., Sotiriou, C., Loi, S., Piccart-Gebhart, M.J., 2013. Pertuzumab: new hope for patients with HER2-positive breast cancer. Ann Oncol 24, 273-282. doi:10.1093/annonc/mds328

Carroll, D.K., Carroll, J.S., Leong, C.-O., Cheng, F., Brown, M., Mills, A.A., Brugge, J.S., Ellisen, L.W., 2006. p63 regulates an adhesion programme and cell survival in epithelial cells. Nat Cell Biol $8,551-$ 561. doi:10.1038/ncb1420

Chaidos, A., Barnes, C.P., Cowan, G., May, P.C., Melo, V., Hatjiharissi, E., Papaioannou, M., Harrington, H., Doolittle, H., Terpos, E., Dimopoulos, M., Abdalla, S., Yarranton, H., Naresh, K., Foroni, L., Reid, A., Rahemtulla, A., Stumpf, M., Roberts, I., Karadimitris, A., 2013. Clinical drug resistance linked to interconvertible phenotypic and functional states of tumor-propagating cells in multiple myeloma.

Blood 121, 318-328. doi:10.1182/blood-2012-06-436220

Chan, A., Delaloge, S., Holmes, F.A., Moy, B., Iwata, H., Harvey, V.J., Robert, N.J., Silovski, T., Gokmen, E., Minckwitz, von, G., Ejlertsen, B., Chia, S.K.L., Mansi, J., Barrios, C.H., Gnant, M., Buyse, M., Gore, I., Smith, J., II, Harker, G., Masuda, N., Petrakova, K., Zotano, A.G., Iannotti, N., Rodriguez, G., Tassone, P., Wong, A., Bryce, R., Ye, Y., Yao, B., Martin, M., 2016. Neratinib after trastuzumabbased adjuvant therapy in patients with HER2-positive breast cancer (ExteNET): a multicentre, randomised, double-blind, placebo-controlled, phase 3 trial. The Lancet Oncology 17, 367-377. doi:10.1016/S1470-2045(15)00551-3 
Chan, S., Scheulen, M.E., Johnston, S., Mross, K., Cardoso, F., Dittrich, C., Eiermann, W., Hess, D., Morant, R., Semiglazov, V., Borner, M., Salzberg, M., Ostapenko, V., Illiger, H.-J., Behringer, D., Bardy-Bouxin, N., Boni, J., Kong, S., Cincotta, M., Moore, L., 2005. Phase II study of Temsirolimus (CCl-779), a novel inhibitor of mTOR, in heavily pretreated patients with locally advanced or metastatic breast cancer. J. Clin. Oncol. 23, 5314-5322. doi:10.1200/JCO.2005.66.130

Chandarlapaty, S., Sawai, A., Scaltriti, M., Rodrik-Outmezguine, V., Grbovic-Huezo, O., Serra, V., Majumder, P.K., Baselga, J., Rosen, N., 2011. AKT inhibition relieves feedback suppression of receptor tyrosine kinase expression and activity. Cancer Cell 19, 58-71. doi:10.1016/j.ccr.2010.10.031

Charafe-Jauffret, E., Ginestier, C., Monville, F., Finetti, P., Adélaïde, J., Cervera, N., Fekairi, S., Xerri, L., Jacquemier, J., Birnbaum, D., Bertucci, F., 2006. Gene expression profiling of breast cell lines identifies potential new basal markers. Oncogene 25, 2273-2284. doi:10.1038/sj.onc.1209254

Chen, E.Y., Tan, C.M., Kou, Y., Duan, Q., Wang, Z., Meirelles, G.V., Clark, N.R., Ma'ayan, A., 2013. Enrichr: interactive and collaborative HTML5 gene list enrichment analysis tool. BMC Bioinformatics 14, 128. doi:10.1186/1471-2105-14-128

Cheng, H., Liu, P., Ohlson, C., Xu, E., Symonds, L., Isabella, A., Muller, W.J., Lin, N.U., Krop, I.E., Roberts, T.M., Winer, E.P., Arteaga, C.L., Zhao, J.J., 2015. PIK3CA(H1047R)- and Her2-initiated mammary tumors escape PI3K dependency by compensatory activation of MEK-ERK signaling. Oncogene. doi:10.1038/onc.2015.377

Cingolani, P., Platts, A., Wang, L.L., Coon, M., Nguyen, T., Wang, L., Land, S.J., Lu, X., Ruden, D.M., 2012. A program for annotating and predicting the effects of single nucleotide polymorphisms, SnpEff: SNPs in the genome of Drosophila melanogaster strain w1118; iso-2; iso-3. Fly (Austin) 6, 80-92. doi:10.4161/fly.19695

Cobleigh, M.A., Langmuir, V.K., Sledge, G.W., Miller, K.D., 2003. A phase I/II dose-escalation trial of bevacizumab in previously treated metastatic breast cancer. Seminars in Oncology 30, 117-124. doi:10.1053/j.seminoncol.2003.08.013

Creighton, C.J., Li, X., Landis, M., Dixon, J.M., Neumeister, V.M., Sjolund, A., Rimm, D.L., Wong, H., Rodriguez, A., Herschkowitz, J.I., Fan, C., Zhang, X., He, X., Pavlick, A., Gutierrez, M.C., Renshaw, L., Larionov, A.A., Faratian, D., Hilsenbeck, S.G., Perou, C.M., Lewis, M.T., Rosen, J.M., Chang, J.C., 2009. Residual breast cancers after conventional therapy display mesenchymal as well as tumor-initiating features. Proc Natl Acad Sci USA 106, 13820-13825. doi:10.1073/pnas.0905718106

Creyghton, M.P., Cheng, A.W., Welstead, G.G., Kooistra, T., Carey, B.W., Steine, E.J., Hanna, J., Lodato, M.A., Frampton, G.M., Sharp, P.A., Boyer, L.A., Young, R.A., Jaenisch, R., 2010. Histone H3K27ac separates active from poised enhancers and predicts developmental state. Proc Natl Acad Sci USA 107, 21931-21936. doi:10.1073/pnas.1016071107

Curtis, C., Shah, S.P., Chin, S.-F., Turashvili, G., Rueda, O.M., Dunning, M.J., Speed, D., Lynch, A.G., Samarajiwa, S., Yuan, Y., Gräf, S., Ha, G., Haffari, G., Bashashati, A., Russell, R., McKinney, S., METABRIC Group, Langerød, A., Green, A., Provenzano, E., Wishart, G., Pinder, S., Watson, P., 
Markowetz, F., Murphy, L., Ellis, I., Purushotham, A., Børresen-Dale, A.-L., Brenton, J.D., Tavaré, S., Caldas, C., Aparicio, S., 2012. The genomic and transcriptomic architecture of 2,000 breast tumours reveals novel subgroups. Nature 486, 346-352. doi:10.1038/nature10983

Danecek, P., Auton, A., Abecasis, G., Albers, C.A., Banks, E., DePristo, M.A., Handsaker, R.E., Lunter, G., Marth, G.T., Sherry, S.T., McVean, G., Durbin, R., 1000 Genomes Project Analysis Group, 2011. The variant call format and VCFtools. Bioinformatics 27, 2156-2158. doi:10.1093/bioinformatics/btr330

De Carvalho, D.D., Sharma, S., You, J.S., Su, S.-F., Taberlay, P.C., Kelly, T.K., Yang, X., Liang, G., Jones, P.A., 2012. DNA Methylation Screening Identifies Driver Epigenetic Events of Cancer Cell Survival. Cancer Cell 21, 655-667. doi:10.1016/j.ccr.2012.03.045

Dean, M., Fojo, T., Bates, S., 2005. Tumour stem cells and drug resistance. Nature Reviews Cancer 5, 275-284. doi:10.1038/nrc1590

Deguchi, K., Ayton, P.M., Carapeti, M., Kutok, J.L., Snyder, C.S., Williams, I.R., Cross, N.C.P., Glass, C.K., Cleary, M.L., Gilliland, D.G., 2003. MOZ-TIF2-induced acute myeloid leukemia requires the MOZ nucleosome binding motif and TIF2-mediated recruitment of CBP. Cancer Cell 3, 259-271.

Dey, A., Chitsaz, F., Abbasi, A., Misteli, T., Ozato, K., 2003. The double bromodomain protein Brd4 binds to acetylated chromatin during interphase and mitosis. Proc Natl Acad Sci USA 100, 8758-8763. doi:10.1073/pnas.1433065100

Dey, N., Williams, C., Leyland-Jones, B., De, P., 2015. A critical role for HER3 in HER2-amplified and non-amplified breast cancers: function of a kinase-dead RTK. American Journal of Translational Research 7, 733-750.

Dickler, M.N., Rugo, H.S., Eberle, C.A., Brogi, E., Caravelli, J.F., Panageas, K.S., Boyd, J., Yeh, B., Lake, D.E., Dang, C.T., Gilewski, T.A., Bromberg, J.F., Seidman, A.D., D'Andrea, G.M., Moasser, M.M., Melisko, M., Park, J.W., Dancey, J., Norton, L., Hudis, C.A., 2008. A phase II trial of erlotinib in combination with bevacizumab in patients with metastatic breast cancer. Clin. Cancer Res. 14, 7878-7883. doi:10.1158/1078-0432.CCR-08-0141

Dillon, S.C., Zhang, X., Trievel, R.C., Cheng, X., 2005. The SET-domain protein superfamily: protein lysine methyltransferases. Genome Biol 6, 227. doi:10.1186/gb-2005-6-8-227

Dreifus, C., 2009. Researcher behind the drug Gleevec. The New York Times.

Duncan, J.S., Whittle, M.C., Nakamura, K., Abell, A.N., Midland, A.A., Zawistowski, J.S., Johnson, N.L., Granger, D.A., Jordan, N.V., Darr, D.B., Usary, J., Kuan, P.-F., Smalley, D.M., Major, B., He, X., Hoadley, K.A., Zhou, B., Sharpless, N.E., Perou, C.M., Kim, W.Y., Gomez, S.M., Chen, X., Jin, J., Frye, S.V., Earp, H.S., Graves, L.M., Johnson, G.L., 2012. Dynamic reprogramming of the kinome in response to targeted MEK inhibition in triple-negative breast cancer. Cell 149, 307-321.

doi:10.1016/j.cell.2012.02.053 
Easwaran, H., Tsai, H.-C., Baylin, S.B., 2014. Cancer epigenetics: tumor heterogeneity, plasticity of stemlike states, and drug resistance. Molecular Cell 54, 716-727. doi:10.1016/j.molcel.2014.05.015

Eckel, K.L., Tentler, J.J., Cappetta, G.J., Diamond, S.E., 2003. The epithelial-specific ETS transcription factor ESX/ESE-1/Elf-3 modulates breast cancer-associated gene expression. DNA and Cell Biology 22, 79-94. doi:10.1089/DNA.2003.22.issue-2;wgroup:string:Publication

Ernst, J., Kellis, M., 2013. Interplay between chromatin state, regulator binding, and regulatory motifs in six human cell types. Genome Research 23, 1142-1154. doi:10.1101/gr.144840.112

Fleming, G.F., Ma, C.X., Huo, D., Sattar, H., Tretiakova, M., Lin, L., Hahn, O.M., Olopade, F.O., Nanda, R., Hoffman, P.C., Naughton, M.J., Pluard, T., Conzen, S.D., Ellis, M.J., 2012. Phase II trial of temsirolimus in patients with metastatic breast cancer. Breast Cancer Res Treat 136, 355-363. doi:10.1007/s10549-011-1910-7

Forozan, F., Veldman, R., Ammerman, C.A., Parsa, N.Z., Kallioniemi, A., Kallioniemi, O.-P., Ethier, S.P., 1999. Molecular cytogenetic analysis of 11 new breast cancer cell lines. $\mathrm{Br}$ J Cancer 81, 1328-1334. doi:10.1038/sj.bjc.6695007

Foulds, C.E., Nelson, M.L., Blaszczak, A.G., Graves, B.J., 2004. Ras/mitogen-activated protein kinase signaling activates Ets-1 and Ets-2 by CBP/p300 recruitment. Mol Cell Biol 24, 10954-10964. doi:10.1128/MCB.24.24.10954-10964.2004

Garrett, J.T., Olivares, M.G., Rinehart, C., Granja-Ingram, N.D., Sanchez, V., Chakrabarty, A., Dave, B., Cook, R.S., Pao, W., McKinely, E., Manning, H.C., Chang, J., Arteaga, C.L., 2011. Transcriptional and posttranslational up-regulation of HER3 (ErbB3) compensates for inhibition of the HER2 tyrosine kinase. Proc Natl Acad Sci USA 108, 5021-5026. doi:10.1073/pnas.1016140108

Garrison, E., Marth, G., 2012. Haplotype-based variant detection from short-read sequencing.

Gaspar-Maia, A., Alajem, A., Polesso, F., Sridharan, R., Mason, M.J., Heidersbach, A., Ramalho-Santos, J., McManus, M.T., Plath, K., Meshorer, E., Ramalho-Santos, M., 2009. Chd1 regulates open chromatin and pluripotency of embryonic stem cells. Nature 460, 863-868. doi:10.1038/nature08212

Gerlinger, M., Rowan, A.J., Horswell, S., Larkin, J., Endesfelder, D., Gronroos, E., Martinez, P., Matthews, N., Stewart, A., Tarpey, P., Varela, I., Phillimore, B., Begum, S., McDonald, N.Q., Butler, A., Jones, D., Raine, K., Latimer, C., Santos, C.R., Nohadani, M., Eklund, A.C., Spencer-Dene, B., Clark, G., Pickering, L., Stamp, G., Gore, M., Szallasi, Z., Downward, J., Futreal, P.A., Swanton, C., 2012. Intratumor Heterogeneity and Branched Evolution Revealed by Multiregion Sequencing. N Engl J Med 366, 883-892. doi:10.1056/NEJMoa1113205

Gradishar, W.J., Yardley, D.A., Layman, R., Sparano, J.A., Chuang, E., Northfelt, D.W., Schwartz, G.N., Youssoufian, H., Tang, S., Novosiadly, R., Forest, A., Nguyen, T.S., Cosaert, J., Grebennik, D., Haluska, P., 2016. Clinical and translational results of a phase II, randomized trial of an anti-IGF-1R (Cixutumumab) in women with breast cancer that progressed on endocrine therapy. Clin. Cancer Res. 22, 301-309. doi:10.1158/1078-0432.CCR-15-0588 
Guenther, M.G., Levine, S.S., Boyer, L.A., Jaenisch, R., Young, R.A., 2007. A chromatin landmark and transcription initiation at most promoters in human cells. Cell 130, 77-88.

doi:10.1016/j.cell.2007.05.042

Hanker, A.B., Pfefferle, A.D., Balko, J.M., Kuba, M.G., Young, C.D., Sanchez, V., Sutton, C.R., Cheng, H., Perou, C.M., Zhao, J.J., Cook, R.S., Arteaga, C.L., 2013. Mutant PIK3CA accelerates HER2driven transgenic mammary tumors and induces resistance to combinations of anti-HER2 therapies. Proc Natl Acad Sci USA 110, 14372-14377. doi:10.1073/pnas.1303204110

Hansen, A.S., Pustova, I., Cattoglio, C., Tjian, R., Elife, X.D., 2017. CTCF and cohesin regulate chromatin loop stability with distinct dynamics. eLife. doi:10.7554/eLife.25776.001

Hata, A.N., Niederst, M.J., Archibald, H.L., Gomez-Caraballo, M., Siddiqui, F.M., Mulvey, H.E., Maruvka, Y.E., Ji, F., Bhang, H.-E.C., Krishnamurthy Radhakrishna, V., Siravegna, G., Hu, H., Raoof, S., Lockerman, E., Kalsy, A., Lee, D., Keating, C.L., Ruddy, D.A., Damon, L.J., Crystal, A.S., Costa, C., Piotrowska, Z., Bardelli, A., lafrate, A.J., Sadreyev, R.I., Stegmeier, F., Getz, G., Sequist, L.V., Faber, A.C., Engelman, J.A., 2016. Tumor cells can follow distinct evolutionary paths to become resistant to epidermal growth factor receptor inhibition. Nat Med 22, 262-269. doi:10.1038/nm.4040

Hehlmann, R., Lauseker, M., Saußele, S., Pfirrmann, M., Krause, S., Kolb, H.J., Neubauer, A., Hossfeld, D.K., Nerl, C., Gratwohl, A., Baerlocher, G.M., Heim, D., Brümmendorf, T.H., Fabarius, A., Haferlach, C., Schlegelberger, B., Müller, M.C., Jeromin, S., Proetel, U., Kohlbrenner, K., Voskanyan, A., Rinaldetti, S., Seifarth, W., Spieß, B., Balleisen, L., Goebeler, M.C., Hänel, M., Ho, A., Dengler, J., Falge, C., Kanz, L., Kremers, S., Burchert, A., Kneba, M., Stegelmann, F., Köhne, C.A., Lindemann, H.W., Waller, C.F., Pfreundschuh, M., Spiekermann, K., Berdel, W.E., Müller, L., Edinger, M., Mayer, J., Beelen, D.W., Bentz, M., Link, H., Hertenstein, B., Fuchs, R., Wernli, M., Schlegel, F., Schlag, R., de Wit, M., Trümper, L., Hebart, H., Hahn, M., Thomalla, J., Scheid, C., Schafhausen, P., Verbeek, W., Eckart, M.J., Gassmann, W., Pezzutto, A., Schenk, M., Brossart, P., Geer, T., Bildat, S., Schäfer, E., Hochhaus, A., Hasford, J., 2017. Assessment of imatinib as first-line treatment of chronic myeloid leukemia: 10-year survival results of the randomized CML study IV and impact of non-CML determinants. Leukemia 31, 2398-2406. doi:10.1038/leu.2017.253

Heintzman, N.D., Hon, G.C., Hawkins, R.D., Kheradpour, P., Stark, A., Harp, L.F., Ye, Z., Lee, L.K., Stuart, R.K., Ching, C.W., Ching, K.A., Antosiewicz-Bourget, J.E., Liu, H., Zhang, X., Green, R.D., Lobanenkov, V.V., Stewart, R., Thomson, J.A., Crawford, G.E., Kellis, M., Ren, B., 2009. Histone modifications at human enhancers reflect global cell-type-specific gene expression. Nature 459, 108112. doi:10.1038/nature07829

Heinz, S., Benner, C., Spann, N., Bertolino, E., Lin, Y.C., Laslo, P., Cheng, J.X., Murre, C., Singh, H., Glass, C.K., 2010. Simple combinations of lineage-determining transcription factors prime cisregulatory elements required for macrophage and B cell identities. Molecular Cell 38, 576-589. doi:10.1016/j.molcel.2010.05.004

Hnisz, D., Day, D.S., Young, R.A., 2016. Insulated Neighborhoods: Structural and Functional Units of Mammalian Gene Control. Cell 167, 1188-1200. doi:10.1016/j.cell.2016.10.024 
Huang, X., Gao, L., Wang, S., McManaman, J.L., Thor, A.D., Yang, X., Esteva, F.J., Liu, B., 2010. Heterotrimerization of the growth factor receptors erbB2, erbB3, and insulin-like growth factor-i receptor in breast cancer cells resistant to herceptin. Cancer Research 70, 1204-1214. doi:10.1158/0008-5472.CAN-09-3321

Hyun, K., Jeon, J., Park, K., Kim, J., 2017. Writing, erasing and reading histone lysine methylations. Exp. Mol. Med. 49, e324-e324. doi:10.1038/emm.2017.11

Infante, J.R., Fecher, L.A., Falchook, G.S., Nallapareddy, S., Gordon, M.S., Becerra, C., DeMarini, D.J., Cox, D.S., Xu, Y., Morris, S.R., Peddareddigari, V.G.R., Le, N.T., Hart, L., Bendell, J.C., Eckhardt, G., Kurzrock, R., Flaherty, K., Burris, H.A., Messersmith, W.A., 2012. Safety, pharmacokinetic, pharmacodynamic, and efficacy data for the oral MEK inhibitor trametinib: a phase 1 dose-escalation trial. The Lancet Oncology 13, 773-781. doi:10.1016/S1470-2045(12)70270-X

Isakoff, S.J., Engelman, J.A., Irie, H.Y., Luo, J., Brachmann, S.M., Pearline, R.V., Cantley, L.C., Brugge, J.S., 2005. Breast cancer-associated PIK3CA mutations are oncogenic in mammary epithelial cells. Cancer Research 65, 10992-11000. doi:10.1158/0008-5472.CAN-05-2612

Jang, M.K., Mochizuki, K., Zhou, M., Jeong, H.-S., Brady, J.N., Ozato, K., 2005. The bromodomain protein Brd4 is a positive regulatory component of P-TEFb and stimulates RNA polymerase IIdependent transcription. Molecular Cell 19, 523-534. doi:10.1016/j.molcel.2005.06.027

Javahery, R., Khachi, A., Lo, K., Zenzie-Gregory, B., Smale, S.T., 1994. DNA sequence requirements for transcriptional initiator activity in mammalian cells. Mol Cell Biol 14, 116-127. doi:10.1128/MCB.14.1.116

Jones, P.A., Takai, D., 2001. The Role of DNA Methylation in Mammalian Epigenetics. Science 293, 1068-1070. doi:10.1126/science.1063852

Jordan, N.V., Prat, A., Abell, A.N., Zawistowski, J.S., Sciaky, N., Karginova, O.A., Zhou, B., Golitz, B.T., Perou, C.M., Johnson, G.L., 2013. SWI/SNF Chromatin-Remodeling Factor Smarcd3/Baf60c Controls Epithelial-Mesenchymal Transition by Inducing Wnt5a Signaling. Mol Cell Biol 33, 30113025. doi:10.1128/MCB.01443-12

Kadoch, C., Crabtree, G.R., 2015. Mammalian SWI/SNF chromatin remodeling complexes and cancer: Mechanistic insights gained from human genomics. Sci Adv 1, e1500447.

doi:10.1126/sciadv.1500447

Keller, P.J., Lin, A.F., Arendt, L.M., Klebba, I., Jones, A.D., Rudnick, J.A., DiMeo, T.A., Gilmore, H., Jefferson, D.M., Graham, R.A., Naber, S.P., Schnitt, S., Kuperwasser, C., 2010. Mapping the cellular and molecular heterogeneity of normal and malignant breast tissues and cultured cell lines. Breast Cancer Res 12, R87. doi:10.1186/bcr2755

Krzywinski, M., Schein, J., Birol, I., Connors, J., Gascoyne, R., Horsman, D., Jones, S.J., Marra, M.A., 2009. Circos: an information aesthetic for comparative genomics. Genome Research 19, 1639-1645. doi:10.1101/gr.092759.109 
Kuleshov, M.V., Jones, M.R., Rouillard, A.D., Fernandez, N.F., Duan, Q., Wang, Z., Koplev, S., Jenkins, S.L., Jagodnik, K.M., Lachmann, A., McDermott, M.G., Monteiro, C.D., Gundersen, G.W., Ma'ayan, A., 2016. Enrichr: a comprehensive gene set enrichment analysis web server 2016 update. Nucleic Acids Res. 44, W90-W97. doi:10.1093/nar/gkw377

Lang, I., Inbar, M.J., Kahán, Z., Greil, R., Beslija, S., Stemmer, S.M., Kaufman, B., Zvirbule, Z., Steger, G.G., Messinger, D., Brodowicz, T., Zielinski, C., 2012. Safety results from a phase III study (TURANDOT trial by CECOG) of first-line bevacizumab in combination with capecitabine or paclitaxel for HER-2-negative locally recurrent or metastatic breast cancer. Eur. J. Cancer 48, 3140-3149. doi:10.1016/j.ejca.2012.04.022

Lee, A., Djamgoz, M.B.A., 2018. Triple negative breast cancer: Emerging therapeutic modalities and novel combination therapies. Cancer Treatment Reviews 62, 110-122. doi:10.1016/j.ctrv.2017.11.003

Lee, C.-K., Shibata, Y., Rao, B., Strahl, B.D., Lieb, J.D., 2004. Evidence for nucleosome depletion at active regulatory regions genome-wide. Nat Genet 36, 900-905. doi:10.1038/ng1400

Liedtke, C., Mazouni, C., Hess, K.R., Andre, F., Tordai, A., Mejia, J.A., Symmans, W.F., GonzalezAngulo, A.M., Hennessy, B., Green, M., Cristofanilli, M., Hortobagyi, G.N., Pusztai, L., 2008. Response to Neoadjuvant Therapy and Long-Term Survival in Patients With Triple-Negative Breast Cancer. Journal of Clinical Oncology 26, 1275-1281. doi:10.1200/JCO.2007.14.4147

Lindsay, J., McDade, S.S., Pickard, A., McCloskey, K.D., McCance, D.J., 2011. Role of $\Delta N p 63 y$ in Epithelial to Mesenchymal Transition. J Biol Chem 286, 3915-3924. doi:10.1074/jbc.M110.162511

Loibl, S., Minckwitz, von, G., Schneeweiss, A., Paepke, S., Lehmann, A., Rezai, M., Zahm, D.M., Sinn, P., Khandan, F., Eidtmann, H., Dohnal, K., Heinrichs, C., Huober, J., Pfitzner, B., Fasching, P.A., Andre, F., Lindner, J.L., Sotiriou, C., Dykgers, A., Guo, S., Gade, S., Nekljudova, V., Loi, S., Untch, M., Denkert, C., 2014. PIK3CA mutations are associated with lower rates of pathologic complete response to anti-human epidermal growth factor receptor 2 (HER2) therapy in primary HER2overexpressing breast cancer. J. Clin. Oncol. 32, 3212-3220. doi:10.1200/JCO.2014.55.7876

Lovén, J., Hoke, H.A., Lin, C.Y., Lau, A., Orlando, D.A., Vakoc, C.R., Bradner, J.E., Lee, T.I., Young, R.A., 2013. Selective Inhibition of Tumor Oncogenes by Disruption of Super-Enhancers. Cell 153, 320-334. doi:10.1016/j.cell.2013.03.036

Ma, C.X., Suman, V.J., Goetz, M., Haluska, P., Moynihan, T., Nanda, R., Olopade, O., Pluard, T., Guo, Z., Chen, H.X., Erlichman, C., Ellis, M.J., Fleming, G.F., 2013. A phase I trial of the IGF-1R antibody Cixutumumab in combination with temsirolimus in patients with metastatic breast cancer. Breast Cancer Res Treat 139, 145-153. doi:10.1007/s10549-013-2528-8

Miller, K.D., Chap, L.I., Holmes, F.A., Cobleigh, M.A., Marcom, P.K., Fehrenbacher, L., Dickler, M., Overmoyer, B.A., Reimann, J.D., Sing, A.P., Langmuir, V., Rugo, H.S., 2005. Randomized phase III 
trial of capecitabine compared with bevacizumab plus capecitabine in patients with previously treated metastatic breast cancer. J. Clin. Oncol. 23, 792-799. doi:10.1200/JCO.2005.05.098

Miller, S.M., Goulet, D.R., Johnson, G.L., 2016. Targeting the Breast Cancer Kinome. J. Cell. Physiol. 232, 53-60. doi:10.1002/jcp.25427

Miller, T.W., Hennessy, B.T., González-Angulo, A.M., Fox, E.M., Mills, G.B., Chen, H., Higham, C., Garcia-Echeverria, C., Shyr, Y., Arteaga, C.L., 2010. Hyperactivation of phosphatidylinositol-3 kinase promotes escape from hormone dependence in estrogen receptor-positive human breast cancer. J. Clin. Invest. 120, 2406-2413. doi:10.1172/JCl41680

Mose, L.E., Wilkerson, M.D., Hayes, D.N., Perou, C.M., Parker, J.S., 2014. ABRA: improved coding indel detection via assembly-based realignment. Bioinformatics 30, 2813-2815. doi:10.1093/bioinformatics/btu376

Mueller, B., Mieczkowski, J., Kundu, S., Wang, P., Sadreyev, R., Tolstorukov, M.Y., Kingston, R.E., 2017. Widespread changes in nucleosome accessibility without changes in nucleosome occupancy during a rapid transcriptional induction. Genes \& Development 31, 451-462. doi:10.1101/gad.293118.116

Murtaza, M., Dawson, S.-J., Tsui, D.W.Y., Gale, D., Forshew, T., Piskorz, A.M., Parkinson, C., Chin, S.F., Kingsbury, Z., Wong, A.S.C., Marass, F., Humphray, S., Hadfield, J., Bentley, D., Chin, T.M., Brenton, J.D., Caldas, C., Rosenfeld, N., 2013. Non-invasive analysis of acquired resistance to cancer therapy by sequencing of plasma DNA. Nature 497, 108-112. doi:10.1038/nature12065

Najafova, Z., Tirado-Magallanes, R., Subramaniam, M., Hossan, T., Schmidt, G., Nagarajan, S., Baumgart, S.J., Mishra, V.K., Bedi, U., Hesse, E., Knapp, S., Hawse, J.R., Johnsen, S.A., 2017. BRD4 localization to lineage-specific enhancers is associated with a distinct transcription factor repertoire. Nucleic Acids Res. 45, 127-141. doi:10.1093/nar/gkw826

Nakajima, N., Horikoshi, M., Roeder, R.G., 1988. Factors involved in specific transcription by mammalian RNA polymerase II: purification, genetic specificity, and TATA box-promoter interactions of TFIID. Mol Cell Biol 8, 4028-4040. doi:10.1128/MCB.8.10.4028

Parker, S.C.J., Stitzel, M.L., Taylor, D.L., Orozco, J.M., Erdos, M.R., Akiyama, J.A., van Bueren, K.L., Chines, P.S., Narisu, N., NISC Comparative Sequencing Program, Black, B.L., Visel, A., Pennacchio, L.A., Collins, F.S., National Institutes of Health Intramural Sequencing Center Comparative Sequencing Program Authors, NISC Comparative Sequencing Program Authors, 2013. Chromatin stretch enhancer states drive cell-specific gene regulation and harbor human disease risk variants. Proc Natl Acad Sci USA 110, 17921-17926. doi:10.1073/pnas.1317023110

Perez, E.A., Romond, E.H., Suman, V.J., Jeong, J.-H., Davidson, N.E., Geyer, C.E., Jr, Martino, S., Mamounas, E.P., Kaufman, P.A., Wolmark, N., 2011. Four-year follow-up of Trastuzumab plus adjuvant chemotherapy for operable human epidermal growth factor receptor 2-positive breast cancer: Joint analysis of data from NCCTG N9831 and NSABP B-31. J. Clin. Oncol. 29, 3366-3373. doi:10.1200/JCO.2011.35.0868 
Perou, C.M., Sørlie, T., Eisen, M.B., van de Rijn, M., Jeffrey, S.S., Rees, C.A., Pollack, J.R., Ross, D.T., Johnsen, H., Akslen, L.A., Fluge, Ø., Pergamenschikov, A., Williams, C., Zhu, S.X., Lønning, P.E., Børresen-Dale, A.-L., Brown, P.O., Botstein, D., 2000. Molecular portraits of human breast tumours. Nature 406, 747-752. doi:10.1038/35021093

Piccart, M., Hortobagyi, G.N., Campone, M., Pritchard, K.I., Lebrun, F., Ito, Y., Noguchi, S., Perez, A., Rugo, H.S., Deleu, I., Burris, H.A., Provencher, L., Neven, P., Gnant, M., Shtivelband, M., Wu, C., Fan, J., Feng, W., Taran, T., Baselga, J., 2014. Everolimus plus exemestane for hormone-receptorpositive, human epidermal growth factor receptor-2-negative advanced breast cancer: overall survival results from BOLERO-2†. Ann Oncol 25, 2357-2362. doi:10.1093/annonc/mdu456

Plotnik, J.P., Budka, J.A., Ferris, M.W., Hollenhorst, P.C., 2014. ETS1 is a genome-wide effector of RAS/ERK signaling in epithelial cells. Nucleic Acids Res. 42, 11928-11940. doi:10.1093/nar/gku929

Polyak, K., Weinberg, R.A., 2009. Transitions between epithelial and mesenchymal states: acquisition of malignant and stem cell traits. Nature Reviews Cancer 9, 265-273. doi:10.1038/nrc2620

Prat, A., Parker, J.S., Karginova, O., Fan, C., Livasy, C., Herschkowitz, J.I., He, X., Perou, C.M., 2010. Phenotypic and molecular characterization of the claudin-low intrinsic subtype of breast cancer. Breast Cancer Res 12, R68. doi:10.1186/bcr2635

Rajagopal, S., Kim, J., Ahn, S., Craig, S., Lam, C.M., Gerard, N.P., Gerard, C., Lefkowitz, R.J., 2010. Beta-arrestin- but not G protein-mediated signaling by the "decoy" receptor CXCR7. Proc Natl Acad Sci USA 107, 628-632. doi:10.1073/pnas.0912852107

Rexer, B.N., Arteaga, C.L., 2012. Intrinsic and acquired resistance to HER2-targeted therapies in HER2 gene-amplified breast cancer: mechanisms and clinical implications. Crit Rev Oncog 17, 1-16.

Rexer, B.N., Ham, A.-J.L., Rinehart, C., Hill, S., de Matos Granja-Ingram, N., Gonzalez-Angulo, A.M., Mills, G.B., Dave, B., Chang, J.C., Liebler, D.C., Arteaga, C.L., 2011. Phosphoproteomic mass spectrometry profiling links Src family kinases to escape from HER2 tyrosine kinase inhibition. Oncogene 30, 4163-4174. doi:10.1038/onc.2011.130

Risom, T., Langer, E.M., Chapman, M.P., Rantala, J., Fields, A.J., Boniface, C., Alvarez, M.J., Kendsersky, N.D., Pelz, C.R., Johnson-Camacho, K., Dobrolecki, L.E., Chin, K., Aswani, A.J., Wang, N.J., Califano, A., Lewis, M.T., Tomlin, C.J., Spellman, P.T., Adey, A., Gray, J.W., Sears, R.C., 2018. Differentiation-state plasticity is a targetable resistance mechanism in basal-like breast cancer. Nat Commun 9, 3815. doi:10.1038/s41467-018-05729-w

Robert, N.J., Diéras, V., Glaspy, J., Brufsky, A.M., Bondarenko, I., Lipatov, O.N., Perez, E.A., Yardley, D.A., Chan, S.Y.T., Zhou, X., Phan, S.-C., O'Shaughnessy, J., 2011. RIBBON-1: randomized, double-blind, placebo-controlled, phase III trial of chemotherapy with or without bevacizumab for firstline treatment of human epidermal growth factor receptor 2-negative, locally recurrent or metastatic breast cancer. Journal of Clinical Oncology 29, 1252-1260. doi:10.1200/JCO.2010.28.0982 
Robinson, D.R., Wu, Y.-M., Vats, P., Su, F., Lonigro, R.J., Cao, X., Kalyana-Sundaram, S., Wang, R., Ning, Y., Hodges, L., Gursky, A., Siddiqui, J., Tomlins, S.A., Roychowdhury, S., Pienta, K.J., Kim, S.Y., Roberts, J.S., Rae, J.M., Van Poznak, C.H., Hayes, D.F., Chugh, R., Kunju, L.P., Talpaz, M., Schott, A.F., Chinnaiyan, A.M., 2013. Activating ESR1 mutations in hormone-resistant metastatic breast cancer. Nat Genet 45, 1446-1451. doi:10.1038/ng.2823

Roeder, R.G., 1996. The role of general initiation factors in transcription by RNA polymerase II. Trends in Biochemical Sciences 21, 327-335. doi:10.1016/S0968-0004(96)10050-5

Rubin, A.J., Barajas, B.C., Furlan-Magaril, M., Lopez-Pajares, V., Mumbach, M.R., Howard, I., Kim, D.S., Boxer, L.D., Cairns, J., Spivakov, M., Wingett, S.W., Shi, M., Zhao, Z., Greenleaf, W.J., Kundaje, A., Snyder, M., Chang, H.Y., Fraser, P., Khavari, P.A., 2017. Lineage-specific dynamic and preestablished enhancer-promoter contacts cooperate in terminal differentiation. Nature Genet 49, 1522-1528. doi:10.1038/ng.3935

Saini, K.S., Loi, S., de Azambuja, E., Metzger-Filho, O., Saini, M.L., Ignatiadis, M., Dancey, J.E., PiccartGebhart, M.J., 2013. Cancer Treatment Reviews. Cancer Treatment Reviews 39, 935-946. doi:10.1016/j.ctrv.2013.03.009

Salazar, N., Muñoz, D., Kallifatidis, G., Singh, R.K., Jordà, M., Lokeshwar, B.L., 2014. The chemokine receptor CXCR7 interacts with EGFR to promote breast cancer cell proliferation. Molecular Cancer 13, 198. doi:10.1186/1476-4598-13-198

Sale, M.J., Cook, S.J., 2014. Intrinsic and acquired resistance to MEK1/2 inhibitors in cancer. Biochem Soc Trans 42, 776-783. doi:10.1042/BST20140129

Selvaraj, N., Kedage, V., Hollenhorst, P.C., 2015. Comparison of MAPK specificity across the ETS transcription factor family identifies a high-affinity ERK interaction required for ERG function in prostate cells. Cell Commun. Signal 13, 12. doi:10.1186/s12964-015-0089-7

Shah, S.P., Roth, A., Goya, R., Oloumi, A., Ha, G., Zhao, Y., Turashvili, G., Ding, J., Tse, K., Haffari, G., Bashashati, A., Prentice, L.M., Khattra, J., Burleigh, A., Yap, D., Bernard, V., McPherson, A., Shumansky, K., Crisan, A., Giuliany, R., Heravi-Moussavi, A., Rosner, J., Lai, D., Birol, I., Varhol, R., Tam, A., Dhalla, N., Zeng, T., Ma, K., Chan, S.K., Griffith, M., Moradian, A., Cheng, S.W.G., Morin, G.B., Watson, P., Gelmon, K., Chia, S., Chin, S.-F., Curtis, C., Rueda, O.M., Pharoah, P.D., Damaraju, S., Mackey, J., Hoon, K., Harkins, T., Tadigotla, V., Sigaroudinia, M., Gascard, P., Tlsty, T., Costello, J.F., Meyer, I.M., Eaves, C.J., Wasserman, W.W., Jones, S., Huntsman, D., Hirst, M., Caldas, C., Marra, M.A., Aparicio, S., 2012. The clonal and mutational evolution spectrum of primary triple-negative breast cancers. Nature 486, 395-399. doi:10.1038/nature10933

Sharma, S.V., Lee, D.Y., Li, B., Quinlan, M.P., Takahashi, F., Maheswaran, S., McDermott, U., Azizian, N., Zou, L., Fischbach, M.A., Wong, K.-K., Brandstetter, K., Ben Wittner, Ramaswamy, S., Classon, M., Settleman, J., 2010. A Chromatin-Mediated Reversible Drug-Tolerant State in Cancer Cell Subpopulations. Cell 141, 69-80. doi:10.1016/j.cell.2010.02.027

Singh, D., Zawistowski, J.S., Stuhlmiller, T.J., Johnson, G.L., n.d. ChIP Peak Analysis. 
Slamon, D.J., Leyland-Jones, B., Shak, S., Fuchs, H., Paton, V., Bajamonde, A., Fleming, T., Eiermann, W., Wolter, J., Pegram, M., Baselga, J., Norton, L., 2009. Use of chemotherapy plus a monoclonal antibody against HER2 for metastatic breast cancer that overexpresses HER2. N Engl J Med 344, 783-792. doi:10.1056/NEJM200103153441101

Sobolik, T., Su, Y.-J., Wells, S., Ayers, G.D., Cook, R.S., Richmond, A., 2014. CXCR4 drives the metastatic phenotype in breast cancer through induction of CXCR2 and activation of MEK and PI3K pathways. Mol. Biol. Cell 25, 566-582. doi:10.1091/mbc.E13-07-0360

Soutourina, J., 2018. Transcription regulation by the Mediator complex. Nature Genet 19, 262-274. doi:10.1038/nrm.2017.115

Stadhouders, R., van den Heuvel, A., Kolovos, P., Jorna, R., Leslie, K., Grosveld, F., Soler, E., 2012. Transcription regulation by distal enhancers: who's in the loop? Transcription 3, 181-186. doi:10.4161/trns.20720

Stratikopoulos, E.E., Dendy, M., Szabolcs, M., Khaykin, A.J., Lefebvre, C., Zhou, M.-M., Parsons, R., 2015. Kinase and BET inhibitors together clamp inhibition of PI3K signaling and overcome resistance to therapy. Cancer Cell 27, 837-851. doi:10.1016/j.ccell.2015.05.006

Stratton, M.R., Campbell, P.J., Futreal, P.A., 2009. The cancer genome. Nature 458, 719-724. doi:10.1038/nature07943

Stuhlmiller, T.J., Miller, S.M., Zawistowski, J.S., Nakamura, K., Beltran, A.S., Duncan, J.S., Angus, S.P., Collins, K.A.L., Granger, D.A., Reuther, R.A., Graves, L.M., Gomez, S.M., Kuan, P.-F., Parker, J.S., Chen, X., Sciaky, N., Carey, L.A., Earp, H.S., Jin, J., Johnson, G.L., 2015. Inhibition of Lapatinibinduced kinome reprogramming in ERBB2-positive breast cancer by targeting BET family bromodomains. Cell Rep 11, 390-404. doi:10.1016/j.celrep.2015.03.037

Suzuki, K., Wilkes, M.C., Garamszegi, N., Edens, M., Leof, E.B., 2007. Transforming growth factor beta signaling via Ras in mesenchymal cells requires p21-activated kinase 2 for extracellular signalregulated kinase-dependent transcriptional responses. Cancer Research 67, 3673-3682. doi:10.1158/0008-5472.CAN-06-3211

Tie, F., Banerjee, R., Stratton, C.A., Prasad-Sinha, J., Stepanik, V., Zlobin, A., Diaz, M.O., Scacheri, P.C., Harte, P.J., 2009. CBP-mediated acetylation of histone H3 lysine 27 antagonizes Drosophila Polycomb silencing. Development 136, 3131-3141. doi:10.1242/dev.037127

Wahba, H.A., El-Hadaad, H.A., 2015. Current approaches in treatment of triple-negative breast cancer. Cancer Biol Med 12, 106-116. doi:10.7497/j.issn.2095-3941.2015.0030

Wang, Y., Waters, J., Leung, M.L., Unruh, A., Roh, W., Shi, X., Chen, K., Scheet, P., Vattathil, S., Liang, H., Multani, A., Zhang, H., Zhao, R., Michor, F., Meric-Bernstam, F., Navin, N.E., 2014. Clonal evolution in breast cancer revealed by single nucleus genome sequencing. Nature 512, 155-160. doi:10.1038/nature13600 
Wani, N.A., Nasser, M.W., Ahirwar, D.K., Zhao, H., Miao, Z., Shilo, K., Ganju, R.K., 2014. C-X-C motif chemokine 12/C-X-C chemokine receptor type 7 signaling regulates breast cancer growth and metastasis by modulating the tumor microenvironment. Breast Cancer Res 16, 1259-17. doi:10.1186/bcr3665

Whyte, W.A., Orlando, D.A., Hnisz, D., Abraham, B.J., Lin, C.Y., Kagey, M.H., Rahl, P.B., Lee, T.I., Young, R.A., 2013. Master transcription factors and mediator establish super-enhancers at key cell identity genes. Cell 153, 307-319. doi:10.1016/j.cell.2013.03.035

Wilkes, M.C., Mitchell, H., Penheiter, S.G., Doré, J.J., Suzuki, K., Edens, M., Sharma, D.K., Pagano, R.E., Leof, E.B., 2005. Transforming growth factor-beta activation of phosphatidylinositol 3-kinase is independent of Smad2 and Smad3 and regulates fibroblast responses via p21-activated kinase-2. Cancer Research 65, 10431-10440. doi:10.1158/0008-5472.CAN-05-1522

Wilson, B.G., Wang, X., Shen, X., McKenna, E.S., Lemieux, M.E., Cho, Y.-J., Koellhoffer, E.C., Pomeroy, S.L., Orkin, S.H., Roberts, C.W.M., 2010. Epigenetic antagonism between polycomb and SWI/SNF complexes during oncogenic transformation. Cancer Cell 18, 316-328. doi:10.1016/j.ccr.2010.09.006

Wolff, A.C., Lazar, A.A., Bondarenko, I., Garin, A.M., Brincat, S., Chow, L., Sun, Y., NeskovicKonstantinovic, Z., Guimaraes, R.C., Fumoleau, P., Chan, A., Hachemi, S., Strahs, A., Cincotta, M., Berkenblit, A., Krygowski, M., Kang, L.L., Moore, L., Hayes, D.F., 2013. Randomized phase III placebo-controlled trial of Letrozole plus oral Temsirolimus as first-line endocrine therapy in postmenopausal women with locally advanced or metastatic breast cancer. J. Clin. Oncol. 31, 195202. doi:10.1200/JCO.2011.38.3331

Wu, P., Nielsen, T.E., Clausen, M.H., 2016. Small-molecule kinase inhibitors: an analysis of FDAapproved drugs. Drug Discov. Today 21, 5-10. doi:10.1016/j.drudis.2015.07.008

Wu, S.-Y., Lee, A.-Y., Lai, H.-T., Zhang, H., Chiang, C.-M., 2013. Phospho switch triggers Brd4 chromatin binding and activator recruitment for gene-specific targeting. Molecular Cell 49, 843-857. doi:10.1016/j.molcel.2012.12.006

Yu, M., Bardia, A., Wittner, B.S., Stott, S.L., Smas, M.E., Ting, D.T., Isakoff, S.J., Ciciliano, J.C., Wells, M.N., Shah, A.M., Concannon, K.F., Donaldson, M.C., Sequist, L.V., Brachtel, E., Sgroi, D., Baselga, J., Ramaswamy, S., Toner, M., Haber, D.A., Maheswaran, S., 2013. Circulating Breast Tumor Cells Exhibit Dynamic Changes in Epithelial and Mesenchymal Composition. Science 339, 580-584. doi:10.1126/science.1228522

Zabidi, M.A., Arnold, C.D., Schernhuber, K., Pagani, M., Rath, M., Frank, O., Stark, A., 2015. Enhancercore-promoter specificity separates developmental and housekeeping gene regulation. Nature 518, 556-559. doi:10.1038/nature13994

Zabidi, M.A., Stark, A., 2016. Regulatory Enhancer-Core- Promoter Communication via Transcription Factors and Cofactors. Trends in Genetics 32, 801-814. doi:10.1016/j.tig.2016.10.003 
Zawistowski, J.S., Bevill, S.M., Goulet, D.R., Stuhlmiller, T.J., Beltran, A.S., Olivares-Quintero, J.F., Singh, D., Sciaky, N., Parker, J.S., Rashid, N.U., Chen, X., Duncan, J.S., Whittle, M.C., Angus, S.P., Velarde, S.H., Golitz, B.T., He, X., Santos, C., Darr, D.B., Gallagher, K., Graves, L.M., Perou, C.M., Carey, L.A., Earp, H.S., Johnson, G.L., 2017. Enhancer Remodeling During Adaptive Bypass to MEK Inhibition Is Attenuated by Pharmacological Targeting of the P-TEFb Complex. Cancer Discovery CD-16-0653. doi:10.1158/2159-8290.CD-16-0653 